\title{
Tax Reform in Two-Sector General Equilibrium
}

\author{
Olivier Cardi, Romain Restout
}

Octobre 2008

GATE Groupe d'Analyse et de Théorie Économique UMR 5824 du CNRS

93 chemin des Mouilles - 69130 Écully - France

B.P. $167-69131$ Écully Cedex

Tél. +33 (0)4 72866060 - Fax +33 (0)4 72866090

Messagerie électronique gate@gate.cnrs.fr

Serveur Web : www.gate.cnrs.fr 


\title{
TAX REFORM IN TWO-SECTOR GENERAL EQUILIBRIUM ${ }^{1}$
}

\author{
Olivier CARDI ${ }^{2}$ and Romain RESTOUT ${ }^{3}$
}

17th October 2008

\begin{abstract}
We use a two-sector open economy model with an imperfectly competitive non traded sector to investigate the dynamic and steady-state effects of three tax reforms: [i] two revenue-neutral tax reforms shifting the tax burden from labor to consumption taxes and [ii] one labor tax reform keeping the marginal tax wedge constant. Regardless of its form, a tax restructuring crowds-in consumption and investment and raises employment. While tax multipliers for overall output are always positive, their size depends on the type of the tax reform and the financing scheme. Interestingly, the trade balance plays a key role in determining the relative size of sectoral tax multipliers: whereas the long-term tax multiplier is always slightly higher in the traded sector than in the non traded sector, this result is reversed in the short-term. Finally, time horizon matters in determining the relationships between both overall and sectoral tax multipliers and labor responsiveness. Keywords: Non Traded Goods; Investment; Employment; Tax Multiplier.
\end{abstract}

JEL Classification: F41; E62; E22; F32.

\footnotetext{
${ }^{1}$ Constructive comments by Mathieu Bussière, Stefan Schubert and Partha Sen are gratefully acknowledged. An earlier version of this paper was presented to the Far Eastern and South Asian Meeting of the Econometric Society held in Singapore, July 16-18th 2008, and the 20th Congress of the European Economic Association held in Milan, August 27-31st 2008, has benefited from very helpful comments of participants.

${ }^{2}$ Corresponding author: Olivier Cardi, ERMES, Université Panthéon-Assas Paris 2. Address: Université Panthéon-Assas Paris 2, 12 Place du Panthéon, 75230 Paris Cedex 05. France. Phone: +33 144418973. Fax: +33140518130. E-mail: olivier.cardi@u-paris2.fr.

${ }^{3}$ ECONOMIX, University Paris X, and GATE, ENS-LSH Lyon. Address: University Paris X, 200 avenue de la République, 92001 Nanterre Cedex. Phone: +33 1409759 63. E-mail: romain.restout@ens-lsh.fr.
} 


\section{Introduction}

Can a tax restructuring have beneficial effects on overall economic performance? Surprisingly, the dynamic general equilibrium literature offers little guidance on what kind of tax reform strategy makers should employ to stimulate employment without depressing consumption and raising the public debt burden, especially in open economy. However, the two last decade have been witness of numerous episodes of fundamental tax reforms in European countries urged to reduce public deficits and unemployment rates. Hence, we address this issue by developing a novel and analytically tractable model model which can be viewed as a two-sector extension of the open economy version of the Baxter and King [1993] dynamic general equilibrium model. ${ }^{1}$ Since tax reforms take various forms, we consider three simple and practicable tax restructuring: two revenue-neutral tax reforms that shift the tax burden from labor to consumption taxes and [ii] one labor tax reform keeping the marginal tax wedge constant. ${ }^{2}$ We show formally that regardless of the type of the tax reform, a tax restructuring crowds-in consumption and investment in the long-term (long-term), raises permanently employment and leads to overall welfare gains.

Urged to reduce high levels of unemployment rates, industrialized countries are tempted to cut labor tax to stimulate employment. However, the unavailability of debt financing restricts the use of such tax policies in numerous OECD economies. In particular, the stability and growth pact is aimed at achieving budget balance in EMU member countries over the medium run. To compensate for the reduction in tax revenue, governments must find other source of tax financing. Since raising lump-sum taxes is a difficult political task, a tax restructuring that is revenue-neutral would be easier to implement. Building on that, a key policy question for European countries is wether a shift of the labor tax burden to consumption tax could be a useful tool for stimulating employment without increasing public debt. Additionally, the type of a tax restructuring should rely upon current countries' tax structure. Table 1 reports the rate of consumption tax $\tau^{c}$, the employers' part of labor taxes $\tau^{F}$ and the employees' part of labor taxes $\tau^{H}$ across thirteen OECD countries over the recent period (2000-2006). ${ }^{3}$ The most striking feature is that both consumption and labor taxes vary considerably across countries and thus there exists a room for a tax reform in several OECD economies. For instance, a strategy which involves simultaneously cutting payroll taxes and raising the consumption tax rate in France and Italy which reached upper bounds of $\tau^{F}$, reducing labor income taxes and increasing $\tau^{c}$ in Belgium, or decreasing $\tau^{F}$ while raising $\tau^{H}$ in Spain.

Would a shift in the level or the composition of the tax wedge be of help in stimulating employment and economic activity? While so far little attention has been paid to such issue, several countries experienced or will experience (in particular East Transition Countries) fundamental tax reforms. From 1986, Denmark gradually moves the tax burden from labor income taxes to consumption taxes; over 1994-1999, Hungary reduces payroll taxes while increasing the effective labor income tax rates; in 2005 Germany cut its payroll taxes while increasing the standard consumption tax rate from $16 \%$ to $19 \%$ in 2007 . This question has triggered an intense debate in the European countries until recently, in particular in France regarding the eventual adoption of the social VAT implemented in Germany. 
Table 1: Consumption and Labor Tax rates in 13 OECD countries

\begin{tabular}{lccclccc}
\hline Countries & $\tau^{c}$ & $\tau^{F}$ & $\tau^{H}$ & Countries & $\tau^{c}$ & $\tau^{F}$ & $\tau^{H}$ \\
\hline Austria & 0.18 & 0.16 & 0.32 & Italy & 0.14 & 0.27 & 0.28 \\
Belgium & 0.13 & 0.20 & 0.42 & Japan & 0.07 & 0.08 & 0.19 \\
Denmark & 0.26 & 0.00 & 0.42 & Netherlands & 0.18 & 0.09 & 0.33 \\
Spain & 0.14 & 0.22 & 0.20 & Sweden & 0.20 & 0.23 & 0.31 \\
Finland & 0.23 & 0.23 & 0.32 & UK & 0.15 & 0.07 & 0.26 \\
France & 0.16 & 0.27 & 0.29 & US & 0.05 & 0.06 & 0.25 \\
Germany & 0.15 & 0.15 & 0.44 & Average & 0.16 & 0.16 & 0.31 \\
\hline Notes: $\tau^{c}:$ consumption tax rate, $\tau^{F}$ : employers' part of labor taxes, and \\
$\tau^{H}:$ employees' part of labor taxes. Source: OECD. Data coverage: 2000- \\
2007
\end{tabular}

There has been recently a growing interest for tax policy, as exemplified by Leeper and Yang [2008], Mankiw and Weinzierl [2006], Trabandt and Uhlig [2006], who consider a closedeconomy model. Instead, Ganelli and Tervala [2008], Lin [1999], Mendoza and Tesar [1998] investigate the implications of a tax reform in an open-economy framework. One major contribution of our paper is to cover both the closed- and open-economy dimensions of contemporaneous industrialized countries by considering both non tradables and tradables. The relevance of the distinction between a non traded sector and a traded sector is supported by the data as shown in Table 2. More specifically, empirical evidence reveal that in OECD countries, the non tradable share in overall GDP and total employment averages to $65 \%$ and about $60 \%$ in total investment expenditure. A second interesting feature is that the response of a two-sector economy to a shock depends heavily on relative sectoral capital intensities. However the two-sector theoretical literature (see e. g. Coto-Martinez and Dixon [2003], Obstfeld [1989]) commonly assumes that the traded sector is more capital intensive for analytical convenience. Our estimation of sectoral output shares of capital income shows that the non traded sector is relatively more capital intensive in 6 of 13 industrialized countries. ${ }^{4}$ Hence, we consider the two cases both analytically and numerically. A third key feature is that our estimation of the markup at a sectoral level shows that the non traded sector displays a large market power. This has been introduced by assuming that the non traded sector is imperfectly competitive.

What are the main economic lessons drawn from our two-sector general equilibrium model? One virtue of the model we developed is that it allows to express the steady-state changes of a tax restructuring as a scaled-down version of long-term effects of a labor tax cut financed by a fall in lump-sum transfers. This enables us to find analytically that regardless of the type of the tax reform, a tax restructuring crowds-in consumption and investment in the long-term, raises permanently employment and thereby boosts overall GDP. To estimate the effectiveness of a tax reform, we calculated both analytically and numerically the long-term and short-term tax multipliers and evaluated the size of overall welfare gains. We find that the tax multipliers for overall output are always positive and agents' overall welfare rises for the benchmark parametrization. Interestingly, adjustments of both investment and trade balance play a key role in determining the effectiveness of a tax restructuring. As the trade balance 
Table 2: Sectoral Capital Intensities, Markups and Non-Tradable Share

\begin{tabular}{lccccccc}
\hline & $\theta^{T}$ & $\theta^{N}$ & $\mu^{N}$ & $\mu^{T}$ & $Y^{N} / Y$ & $L^{N} / L$ & $I^{N} / I$ \\
\hline France & 0.27 & 0.30 & 1.42 & 1.22 & 0.69 & 0.64 & 0.63 \\
Germany & 0.22 & 0.35 & 1.55 & 1.17 & 0.64 & 0.61 & 0.61 \\
Italy & 0.22 & 0.33 & 1.73 & 1.24 & 0.63 & 0.56 & 0.54 \\
Japan & 0.42 & 0.39 & 1.63 & 1.28 & 0.64 & 0.61 & 0.59 \\
US & 0.36 & 0.32 & 1.42 & 1.24 & 0.68 & 0.72 & 0.59 \\
Average $(13)$ & 0.32 & 0.32 & 1.48 & 1.23 & 0.65 & 0.64 & 0.59 \\
$k^{t}$ raded $>k^{n}$ ontraded (6) & 0.37 & 0.31 & 1.44 & 1.26 & 0.64 & 0.64 & 0.60 \\
$k^{n}$ ontraded $>k^{t}$ raded (5) & 0.28 & 0.33 & 1.51 & 1.21 & 0.66 & 0.63 & 0.57 \\
\hline \multicolumn{6}{c}{ Notes: $\theta^{T}\left(\theta^{N}\right.$ respectively): output share of capital income in the traded (non } \\
$\quad$ traded respectively) sector. $\mu^{T}$ and $\mu^{N}$ are the markups charged in the traded \\
$\quad$ and non traded sector. $Y^{N} / Y, L^{N} / L$ and $I^{N} / I$ are the non tradable share \\
\multicolumn{7}{l}{ in overall GDP, total employment and investment expenditures respectively. } \\
Source: OECD, EU KLEMS. Data coverage: 1970-2004.
\end{tabular}

enters on deficit on impact, the short-term tax multiplier which falls in the range 0.03-0.14 is always smaller than the long-term tax multiplier which comprises between 0.05 and 0.25 . More interestingly, time horizon matters in determining the size of sectoral expansionary effects. Whereas its share in overall output is about $1 / 3$ in industrialized countries, the boom in the traded sector in the long-term amounts for half of GDP increase. Unlike, in the short-term, output in the traded sector drops dramatically while the non traded sector expands sizeably.

One important contribution of our formal study is to highlight the roles of two critical parameters in determining the size of the tax multiplier: the elasticity of labor supply and the degree of tax progressiveness. Hence, we conducted a sensitivity analysis with respect to these to parameters. First, like Baxter and King [1993], we find that the long-term tax multiplier for overall output rises monotonically with labor responsiveness. Unlike, the shortterm tax multiplier decreases as long as the elasticity labor supply exceeds unity since the depressing effect on the domestic demand induced by the higher consumption price index predominates over the stimulating effect originating from the standard wealth effect. We get a more complete picture by conducting the sensitivity analysis at a sectoral level. We find that the short-term sectoral tax multiplier in the non traded sector rises and in the traded sector falls as labor supply gets more responsive since investment is crowded-in further by the tax reform. Finally, the long-term tax multiplier falls or rises with the degree of tax progressiveness depending on wether the tax restructuring keeps the tax revenue or the tax wedge constant since in the latter case, the depressing effect induced by the rise in the labor income tax gets smaller as the tax scheme gets more progressive.

The paper is organized as follows. Section 2 outlines the specification of a two-sector model where the traded good is produced by a perfectly competitive sector and the non traded good is produced by an imperfectly competitive sector. In sections 3 and 4, we discuss the steady-state and the dynamic effects of three tax restructuring. Section 3 focuses on tax rate changes when lump-sum transfers are used to balance the government budget. Section 4 analyzes the implications of alternative revenue-neutral tax restructuring scenarios. In 
section 5, we conduct a sensitivity analysis at an overall and a sectoral level with respect to key parameters. Section 6 concludes.

\section{The Framework}

Consider a small open economy that is populated by a constant number of identical households and firms that have perfect foresight and live forever. The country is small in world goods and capital markets and faces given world interest rate, $r^{\star}$. A perfectly competitive sector produces a traded good denoted by the superscript $T$ that can be exported and consumed domestically. An imperfectly competitive sector produces a non traded good denoted by the superscript $N$ which is devoted to physical capital accumulation and domestic consumption. Finally, the traded good is chosen as numeraire.

\subsection{Households}

At each instant the representative agent consumes traded goods and non traded goods denoted respectively by $c^{T}$ and $c^{N}$. We assume that there is a continuum of commodities indexed by the subscript $j \in[0, n]$ and denoted by $\mathrm{c}^{N}(j)$, each produced by a different firm. Consumption of non traded commodity $j$ is aggregated by the means of the following formulation: ${ }^{5}$

$$
c^{N}=n^{\frac{1}{1-\sigma}}\left[\int_{0}^{n} \mathrm{c}^{N}(j)^{\frac{\sigma-1}{\sigma}}\right]^{\frac{\sigma}{\sigma-1}}, \quad \sigma>1,
$$

where the size of the parameter $\sigma$ reflects the ease with which any varieties can be substituted for each other. Denoting by $p$ the unit cost of the non traded composite good and using costminimizing, the demand for variety $j$ of the non traded consumption good can be written as follows:

$$
c^{N}(j)=\frac{1}{n}\left(\frac{\varrho(j)}{p}\right)^{-\sigma} c^{N}, \quad p=n^{-\frac{1}{1-\sigma}}\left[\int_{0}^{n} \varrho(j)^{1-\sigma}\right]^{\frac{1}{1-\sigma}},
$$

where $c^{N}$ represents the composite non traded consumption good.

The agent is endowed with a unit of time and supplies a fraction $L(t)$ as labor and the remainder, $l \equiv 1-L$ is consumed as leisure. At any instant of time, households derive utility from their consumption and leisure (and thus a disutility from labor supply). The traded and the non traded good are aggregated by a constant elasticity of substitution function:

$$
c\left(c^{T}, c^{N}\right)=\left[\varphi^{\frac{1}{\phi}}\left(c^{T}\right)^{\frac{\phi-1}{\phi}}+(1-\varphi)^{\frac{1}{\phi}}\left(c^{N}\right)^{\frac{\phi-1}{\phi}}\right]^{\frac{\phi}{\phi-1}},
$$

with $\varphi$ the weight attached to the traded good in the overall consumption bundle $(0<\varphi<1)$ and $\phi$ the intratemporal elasticity of substitution $(\phi>0)$.

The representative household maximizes a lifetime utility function:

$$
U=\int_{0}^{\infty}\left\{u\left[c\left(c^{T}(t), c^{N}(t)\right)\right]+v(L)\right\} e^{-\beta t} \mathrm{~d} t,
$$


where $\beta$ is the consumer's discount rate. For analytical simplicity, instantaneous utility function $\xi \equiv u(c)+v(L)$ is assumed to be separable in consumption and labor and to take an iso-elastic form:

$$
\xi \equiv \frac{1}{1-\frac{1}{\sigma_{c}}} c^{1-\frac{1}{\sigma_{c}}}-\gamma \frac{1}{1+\frac{1}{\sigma_{L}}} L^{1+\frac{1}{\sigma_{L}}}
$$

where $\sigma_{L}>0$ is the Frisch elasticity of labor supply (or intertemporal elasticity of substitution for labor supply); $\gamma>0$ is a simple scaling factor which parametrizes the magnitude of disutility from working. 6

Households supply $L(t)$ units of labor services and hold the physical capital stock for which they receive the capital rental rate. In addition, they accumulate internationally traded bonds holding, $b(t)$, that yields net interest rate earnings $r^{\star} b(t)$, expressed in terms of the traded good. Denoting by $w^{A} L$ the after-tax labor income and lump-sum transfers from the State by $Z$, the flow budget constraint writes as follows:

$$
\dot{b}(t)=r^{\star} b(t)+\left(r^{K}+\delta_{K}\right) K(t)+w^{A} L(t)-p_{c}(p(t))\left(1+\tau^{c}\right) c(t)-p(t) I(t)+Z .
$$

The flow budget constraint states that households receive the gross real wage $w$ and pay a labor income tax $\tau^{H}$ above a certain threshold denoted by $\kappa$. Thus, $w^{A}=w-(w-\kappa) \tau^{H}$ corresponds to the after-tax "average" wage and $w^{H}=w\left(1-\tau^{H}\right)$ denotes the after-tax "marginal" wage. As longer as tax allowances are positive, the tax system is progressive which means that the average tax burden rises with the wage rate. Households devote their financial and non financial wealth to expenditure in consumption and investment goods.

Total expenditure in consumption goods is equal to $p_{c}\left(1+\tau^{c}\right) c$ with $\tau^{c}$ the consumption tax rate and $p_{c}$ the consumption price index. Households accumulate $p I$ with $p$ the relative price of the non traded good in terms of the traded good. We assume that $I$ is defined analogously (all investment consists in non traded goods) to $c^{N}$ with the same elasticity of substitution $\sigma$ :

$$
I=n^{\frac{1}{1-\sigma}}\left[\int_{0}^{n} \iota(j)^{\frac{\sigma-1}{\sigma}}\right]^{\frac{\sigma}{\sigma-1}}, \quad \sigma>1,
$$

Adopting a similar cost-minimizing procedure than we used for consumption, households' demand for variety $j$ of the investment good is thus given by:

$$
i(j)=\frac{1}{n}\left(\frac{\varrho(j)}{p}\right)^{-\sigma} I .
$$

Since $c^{N}$ and $I$ feature the same function formal (i. e. an identical elasticity of demand), the price index for investment good is given by $p$ (see (2)). Aggregate investment gives rise to overall capital accumulation according to the following dynamic equation:

$$
\dot{K}(t)=I(t)-\delta_{K} K(t)
$$

where we assume that physical capital depreciates at rate $\delta_{K}$.

Since $c($.$) is homothetic, the household's maximization problem can be decomposed into$ two stages. In the first stage, consumers choose their real consumption, $c$, labor supply $L$, and the rates of accumulation of traded bonds together with domestic capital to maximize (4) 
subject to the flow budget constraint (6) and initial conditions $b(0)=b_{0}$ and $K(0)=K_{0}$. At the second stage, the cost-minimizing intratemporal allocation between non traded and traded goods follows immediately from the Shephard's Lemma: $p c^{N}=\alpha_{c} p_{c} c$ and $c^{T}=\left(1-\alpha_{c}\right) p_{c} c$, with $\alpha_{c}$ the share of non traded goods in consumption expenditure.

\section{$2.2 \quad$ Firms}

Both sectors use physical capital, $K^{T}$ and $K^{N}$, and labor, $L^{T}$ and $L^{N}$, according to a constant returns to scale production function, $Y^{T}=F\left(K^{T}, L^{T}\right)$ and $Y^{N}=H\left(K^{N}, L^{N}\right)$, which are assumed to have the usual neoclassical properties of positive and diminishing marginal products. The traded and non traded sectors face two cost components: a capital rental cost equal to $r^{K}+\delta_{K}$, and a labor cost equal to $w^{F}=w\left(1+\tau^{F}\right)$ with $\tau^{F}$ denotes the payroll tax rate (i. e the employer's part of labor taxes).

The first order conditions derived from profit-maximization in the traded sector state that capital marginal product is equal to capital rental rate, i. e. $F_{K}=r^{K}+\delta_{K}$, and labor marginal product equalizes labor cost, i. e. $F_{L}=w^{F}$. Firm $j$ in the non traded sector produces a level of output $Z^{N}(j)=Y^{N}(j)-F C(j)$ with $Y^{N}(j)=H\left(\mathcal{K}^{N}(j), \mathcal{L}^{N}(j)\right)$ and $F C(j)>0$ is a fixed cost. Each firm $j$ chooses capital and labor to maximize profits subject to the demand for each brand originating from the private $c^{N}(j)+\iota(j)$ and the public sector $g^{N}(j)$, taking the price index $p$ for the composite consumption good and input prices as given:

$$
\begin{aligned}
& \varrho(j) H\left(\mathcal{K}^{N}(j), \mathcal{L}^{N}(j)\right)-\left(r^{K}+\delta_{K}\right) \mathcal{K}^{N}(j)-w^{F} \mathcal{L}^{N}(j)-\varrho(j) F C(j), \\
& \text { subject to } Y^{N}(j)=c^{N}(j)+\iota(j)+g^{N}(j),
\end{aligned}
$$

which assumes that labor is perfectly mobile across firms so that all firms are constrained to pay the same wage $w^{F}$. This choice problem for firm $j$ yields the conditions that the marginal revenue of capital $\frac{\varrho(j) H_{K}}{\mu}$ and labor $\frac{\varrho(j) H_{L}}{\mu}$ equalizes the capital rental rate $r^{K}+\delta_{K}$ and the producer wage $w^{F}$. The marginal product of each input is lowered by the (constant) markup $\mu\left(=\mu^{N}\right)$ which defined as follows:

$$
\mu \equiv \frac{\sigma}{\sigma-1}
$$

with $\sigma>1$ the elasticity of demand facing firm $j$. Since commodities are differentiated, each firm has market power and is able to set a price over the marginal cost. In this paper, we further assume that free entry drives profits down to zero, i. e. $\varrho(j) H\left(\mathcal{K}^{N}(j), \mathcal{L}^{N}(j)\right)-$ $r^{K} \mathcal{K}^{N}(j)-w^{F} \mathcal{L}^{N}(j)-\varrho(j) F C(j)=0$ for all $j$. In a free entry equilibrium, a proportion $\frac{1}{\sigma}$ of total revenue will cover fixed cost (since $1-\frac{1}{\sigma}$ always covers variable cost).

We should focus on a symmetric equilibrium where all non competitive firms will produce the same output and will charge the same price $\varrho(j)=\varrho=p$. Under symmetry, aggregate consumption and investment in the non traded good are $c^{N}=n \mathrm{c}^{N}$ and $I=n \iota$. In addition, we have the resource constraints $L^{T}+L^{N}=L$ with $L^{N}=n \mathcal{L}^{N}$ and $K^{T}+K^{N}=K$ with $K^{N}=n \mathcal{K}^{N}$. With free-entry, the zero-profit condition determines the number of firms which is given by $n F C=(\mu-1) Z^{N}$, using the fact that $Z^{N}=Y^{N} / \mu{ }^{7}$ 


\subsection{Government}

The final agent in the economy is the government who finances lump-sum transfers to households $Z$ together with public spending falling on the traded $g^{T}$ and the non traded good $p g^{N}$ by raising taxes on consumption, $\tau^{c} p_{c} c$, labor, $\left[\tau^{H}(w-\kappa)+\tau^{F} w\right]$ according to the following balanced condition:

$$
\tau^{c} p_{c} c+\left[\tau^{H}(w-\kappa)+\tau^{F} w\right] L=Z+g^{T}+p g^{N},
$$

where labor taxes can be rewritten as $\left(w^{F}-w^{A}\right) L$. Similar to consumption, government spending in the composite non traded good $g^{N}$ is defined analogously to $c^{N}$ and $I$.

\subsection{Macroeconomic Equilibrium and Equilibrium Dynamics}

Denoting by $k^{i} \equiv K^{i} / L^{i}$ the capital-labor ratio for sector $i=T, N$, we express the production functions in intensive form, that is $y^{T}=f\left(k^{T}\right) \equiv F\left(K^{T}, L^{T}\right) / L^{T}$ and $y^{N}=h\left(k^{N}\right) \equiv$ $H\left(K^{N}, L^{N}\right) / L^{N}$, where small letters mean that the variable is expressed in terms of the sector specific labor.

To obtain the macroeconomic equilibrium, we first derive the optimality conditions for households and firms and then combine these with the inputs allocation constraints and accumulation equations. The macroeconomic equilibrium is summarized by the following set of equations:

$$
\begin{gathered}
u_{c}(c)=p_{c}(p)\left(1+\tau^{c}\right) \lambda, \\
v_{L}(L)=-\lambda w^{A}, \\
f_{k}\left(k^{T}\right)=\frac{p}{\eta} h_{k}\left(k^{N}\right) \equiv r^{K}+\delta_{K} \\
{\left[f\left(k^{T}\right)-k^{T} f_{k}\left(k^{T}\right)\right]=\frac{p}{\eta}\left[h\left(k^{N}\right)-k^{N} h_{k}\left(k^{N}\right)\right] \equiv w\left(1+\tau^{F}\right),} \\
L^{T} k^{T}+L^{N} k^{N}=K \\
L^{T}+L^{N}=L, \\
\dot{\lambda}=\lambda\left(\beta-r^{\star}\right), \\
\frac{r^{K}}{p}+\frac{\dot{p}}{p}=r^{\star} \\
\frac{1}{\mu} Y^{N}=c^{N}+I+g^{N} \\
\dot{b}=r^{\star} b(t)+Y^{T}-c^{T}-g^{T}
\end{gathered}
$$

and dynamic equation (9) and the transversality conditions:

$$
\lim _{t \rightarrow \infty} p(t) K(t) e^{-r^{\star} t}=\lim _{t \rightarrow \infty} b(t) e^{-r^{\star} t}=0 .
$$

$\lambda$ is the co-state variables associated with dynamic equation (6). We require the time preference rate to be equal to the world interest rate:

$$
\beta=r^{\star},
$$


in order to generate an interior solution. This standard assumption made in the literature implies that the marginal utility of wealth, $\lambda$, must remain constant over time, i. e. $\lambda=\bar{\lambda}$ (see e. g. Cardi [2007] on that subject). This gives rise to the zero-root property which has the appealing feature to allow for splitting the steady-state changes into a wealth effect and a tax effect.

Static efficiency conditions (13a) and (13b) can be solved for real consumption and labor which of course must hold at any point of time:

$$
c=c\left(\bar{\lambda}, p, \tau^{c}\right), \quad L=L\left(\bar{\lambda}, p, \tau^{F}, \tau^{H}\right),
$$

with $c_{\bar{\lambda}}<0, c_{p}<0, c_{\tau^{c}}<0, L_{\bar{\lambda}}>0$; additionally, $L_{\tau^{F}}<0, L_{\tau^{H}}<0$, and $L_{p} \lessgtr 0$ according to wether $k^{N} \gtrless k^{T}$. A rise in agent's wealth (i. e. a fall in the marginal utility of wealth $\bar{\lambda}$ ) stimulates consumption while discouraging labor supply. A rise in the relative price of non tradables $p$ reduces real consumption. An increase in $p$ decreases or increases the wage rate $w$ and thereby lowers or raises labor supply according to wether $k^{N} \gtrless k^{T}$. While a rise in the consumption tax rate $\tau^{c}$ depresses consumption by raising its marginal cost, a fall in the labor income tax rate levied on employers $\tau^{F}$ or employees $\tau^{H}$ stimulates labor supply by raising the after-tax labor income.

Static efficiency conditions (13c)-(13d) state sectoral marginal revenue products must equalize to the labor producer cost $w^{F}$ and capital rental rate $r^{K}+\delta_{K}$. They can be solved for sector capital intensities ratios:

$$
k^{T}=k^{T}(p), \quad k^{N}=k^{N}(p),
$$

where the signs of other partial derivatives are dependent upon relative sectoral capital intensities: $k_{p}^{T} \lessgtr 0, k_{p}^{N} \lessgtr 0$ according to wether $k^{N} \gtrless k^{T}$. Substituting (17) into the resource constraints for capital and labor (13e)-(13f), we obtain the static solutions for traded and the non traded labor; then we solve for the traded and the non traded output: ${ }^{8}$

$$
Y^{T}=Y^{T}\left(K, p, \bar{\lambda}, \tau^{F}, \tau^{H}\right), \quad Y^{N}=Y^{N}\left(K, p, \bar{\lambda}, \tau^{F}, \tau^{H}\right),
$$

where $Y_{K}^{T} \lessgtr 0, Y_{\bar{\lambda}}^{T} \gtrless 0, Y_{K}^{N} \gtrless 0, Y_{\bar{\lambda}}^{N} \lessgtr 0, Y_{\tau^{j}}^{T} \lessgtr 0$ and $Y_{\tau^{j}}^{N} \gtrless 0$ (with $j=H, F$ ) depending on wether $k^{N} \gtrless k^{T}$, together with $Y_{p}^{T}<0$ and $Y_{p}^{N}>0$. A rise in aggregate capital stock $K$ induces a shift of labor in the direction of the more capital-intensive sector. A rise in total employment triggered by a rise in $\bar{\lambda}$ or a labor tax cut $\tau^{j}(j=F, H)$ shifts labor in favor of the most labor-intensive sector. An appreciation in the real exchange rate attracts resources from the traded to the non traded sector.

The transitional adjustment of the two-sector economy is driven by three equations (13h),(13i) and (13j). The two dynamic equations (13h)-(13i) form a separate subsystem in $p$ and $K$. The capital accumulation equation (13i) which states that investment must eliminate an eventual excess demand or excess supply in the non traded good market. The dynamic equation for the real exchange rate $(13 \mathrm{~h})$ adjusts to equalize the returns on domestic capital, i. e. $\frac{r^{K}}{p}+\frac{\dot{p}}{p}=\frac{h_{k}}{\mu}-\delta_{K}+\frac{\dot{p}}{p}$, and foreign assets $r^{\star}$. Since the system features one state variable, $K$, and one jump variable, $p$, the equilibrium yields a unique one-dimensional stable 
saddle-path, irrespective of the relative sizes of capital-labor ratios. Starting from an initial aggregate capital stock $K(0)=K_{0}$, the stable dynamic time paths followed by $K$ and $p$ write as follows:

$$
K(t)=\tilde{K}+\left(K_{0}-\tilde{K}\right) e^{\nu_{1} t}, \quad p(t)=\tilde{p}+\omega_{2}^{1}(K(t)-\tilde{K}),
$$

where $\omega_{2}^{1}<0$ is the eigenvector associated with the stable eigenvalue $\nu_{1}<0$ and we normalized the eigenvector $\omega_{1}^{1}$ to unity.

Equation (13j) states that the current account surplus, which comprises the trade balance, must be equal to the value of output less absorption $\left(c^{T}+g^{T}\right)$, plus interest payments on outstanding assets. ${ }^{9}$ Substituting first the ST solutions (16) and (18), then linearizing the dynamic equation of the internationally traded bonds (13j) in the neighborhood of the steadystate, substituting the solutions for $K(t)$ and $p(t)$ and finally invoking the transversality condition, one obtain the linearized version of the nation's intertemporal budget constraint:

$$
\left(b_{0}-\tilde{b}\right)=\Phi_{1}\left(K_{0}-\tilde{K}\right)
$$

where the initial stocks $K_{0}$ and $b_{0}$ are given. The stable solution for $b(t)$ consistent with LT solvency writes as follows:

$$
(b(t)-\tilde{b})=\Phi_{1}(K(t)-\tilde{K}),
$$

where $\Phi_{1}$ describes the instantaneous effect of capital accumulation on the current account.

As emphasized by Turnovsky and Sen [1995], a striking feature of the transitional adjustment of the economy is its dependency upon the relative capital intensities of sectors $T$ and $N$. If $k^{T}>k^{N}$, we have $\omega_{2}^{1}=0$ and $\Phi_{1}=-\tilde{p}<0$. Thus, the real exchange rate dynamics degenerate, savings is unaffected and current account mirrors capital investment flow. Unlike, with the reversal of capital intensities, $\omega_{2}^{1}<0$ and $\Phi_{1}<0 .{ }^{10}$ Along the transitional path, overall capital stock and the relative price of non tradables move in opposite direction. ${ }^{11}$

\subsection{Steady-State}

Substituting first the ST static solutions (16), (17) and (18), the steady-state of the economy is obtained by setting $\dot{K}, \dot{p}, \dot{b}=0$ and is defined by the following set of equations:

$$
\begin{gathered}
\frac{h_{k}\left[k^{N}(\tilde{p})\right]}{\mu}-\delta_{K}=r^{\star}, \\
\frac{1}{\mu} Y^{N}\left(\tilde{K}, \tilde{p}, \bar{\lambda}, \tau^{F}, \tau^{H}\right)-c^{N}\left(\bar{\lambda}, \tilde{p}, \tau^{c}\right)-\delta_{K} \tilde{K}-g^{N}=0, \\
r^{\star} \tilde{b}+Y^{T}\left(\tilde{K}, \tilde{p}, \bar{\lambda}, \tau^{F}, \tau^{H}\right)-c^{T}\left(\bar{\lambda}, \tilde{p}, \tau^{c}\right)-g^{T}=0,
\end{gathered}
$$

and the intertemporal solvency condition

$$
\left(b_{0}-\tilde{b}\right)=\Phi\left(K_{0}-\tilde{K}\right)
$$

The steady-state equilibrium composed by these four equations jointly determine $\tilde{p}, \tilde{K}, \tilde{b}$ and $\bar{\lambda}$. 
Equation (22a) asserts the rate of return on domestic capital income is equal to the exogenous world interest rate, $r^{\star}$. Since the world interest rate is fixed, this equality determines the steady-state values of $p$ which thereby is unaffected by a tax restructuring. Equation $(22 \mathrm{~b})$ restates that the production of non traded goods net of fixed cost must be exactly outweighed by a demand counterpart. The market clearing condition at the steady-state determines the LT level of the capital stock for given marginal utility of wealth. This LT capital stock requires that an amount $\delta_{K} \tilde{K}$ be invested. Equation (22c) implies that in the steady-state equilibrium, the current account must be zero which means that interest receipts from foreign assets holding, i. e. $r^{\star} \tilde{b}$, must be equal to net exports denoted by $\tilde{n x}$ with $n x=Y^{T}-c^{T}-g^{T}$.

System (22) may be solved for the steady-state values by applying a two-step procedure. We first solve equations (22a) to (22c) as functions of the marginal utility of wealth, $\bar{\lambda}$, and tax parameters, $\tau^{c}, \tau^{F}$ and $\tau^{H}:^{12}$

$$
\tilde{K}=K\left(\bar{\lambda}, \tau^{F}, \tau^{H}, \tau^{c}\right), \quad \tilde{b}=v\left(\bar{\lambda}, \tau^{F}, \tau^{H}, \tau^{c}\right),
$$

with $K_{\bar{\lambda}}>0, K_{\tau^{c}} \lessgtr 0$ depending on wether $k^{N} \gtrless k^{T}, K_{\tau^{H}}<0, K_{\tau^{F}}<0, v_{\bar{\lambda}}<0, v_{\tau^{c}}<0$, $v_{\tau^{F}}>0$, and $v_{\tau^{H}}>0 .{ }^{13}$

The signs of partial derivatives of steady-state functions merit comments. An increase in the marginal utility of wealth, reflecting the wealth effect, induces agents to reduce their real consumption and raise labor supply. In the case $k^{N}>k^{T}$ (resp. $k^{T}>k^{N}$ ), an excess demand (resp. supply) arises in the non traded good market which requires a higher capital stock for the non traded goods market-clearing condition to hold. For the current account to be zero in the LT, higher exports must be exactly matched by lower net interest receipts from traded bonds holding.

A rise of consumption tax $\tau^{c}$ or $\tau^{j}(j=F, H)$ reflects the tax effect which influences the consumption and labor supply of households. If $k^{N}>k^{T}$ (resp. $k^{T}>k^{N}$ ), an increase in $\tau^{c}$ depresses demand for non tradables which in turn calls for capital decumulation (accumulation). Regardless of sectoral capital-labor ratios, since the capital adjustment raises the output of the traded sector and the rise in $\tau^{c}$ reduces the domestic demand for that good, the trade balance improves which in turn requires a LT fall in $b$. A cut in the labor $\operatorname{tax} \tau^{j}$ $(j=F, H)$ lowers the after-tax labor income $w^{A}$ which induces agents to supply more labor. If $k^{N}>k^{T}$ (resp. $k^{T}>k^{N}$ ), the rise in employment depresses (stimulates) the production of non traded goods. The excess demand (resp. supply) in the non traded good market which is eliminated by the rise in the capital stock. Regardless of sectoral capital intensities, the accumulation of physical capital leads to a decumulation of foreign bonds.

\section{Lump-Sum Transfer Financing and Tax Rate Changes}

Since we explore tax reform strategies which require simultaneous changes of two tax rates in section 4, we found convenient to discuss briefly the steady-state effects of a labor tax cut and a rise in the consumption tax rate separately, for pedagogical purpose. Qualitative and 
quantitative responses of main macroeconomic variables are reported in Table 3. Note that the change of tax rate are financed by a change in lump-sum transfers.

Table 3: Steady-State Effects of a Rise in Consumption Tax and a Fall in Payroll Tax

\begin{tabular}{|c|c|c|c|c|c|c|c|c|}
\hline & \multirow{2}{*}{\multicolumn{2}{|c|}{ Model }} & \multicolumn{6}{|c|}{ Numerical } \\
\hline & & & \multicolumn{3}{|c|}{$k^{T}>k^{N}$} & \multicolumn{3}{|c|}{$k^{N}>k^{T}$} \\
\hline & $\mathrm{d} \tau^{c}>0$ & $\mathrm{~d} \tau^{j}<0$ & $\mathrm{~d} \tau^{c}>0$ & $\mathrm{~d} \tau^{F}<0$ & $\mathrm{~d} \tau^{H}<0$ & $\mathrm{~d} \tau^{c}>0$ & $\mathrm{~d} \tau^{F}<0$ & $\mathrm{~d} \tau^{H}<0$ \\
\hline$c$ & - & + & -0.98 & 0.96 & 1.11 & -0.96 & 0.89 & 1.29 \\
\hline$L$ & - & + & -0.89 & 0.87 & 1.01 & -0.86 & 0.80 & 1.15 \\
\hline$K$ & - & + & -0.93 & 0.91 & 1.05 & -0.82 & 0.76 & 1.10 \\
\hline$b$ & + & - & 5.08 & -4.95 & -5.73 & 3.41 & -3.18 & -4.58 \\
\hline$Y$ & - & + & -0.91 & 0.88 & 1.02 & -0.85 & 0.79 & 1.13 \\
\hline
\end{tabular}
after the consumption tax rise $\left(\mathrm{d} \tau^{c}=+0.03\right)$ and income tax cuts $\left(\mathrm{d} \tau^{F}\right.$ and $\left.\mathrm{d} \tau^{H}=-0.03\right)$.

\section{Benchmark Parametrization}

The model is calibrated for a plausible set of utility and production parameters in order to be consistent with data of OECD countries (see Table 4 for a summary). The production functions are supposed to take a Cobb-Douglas form:

$$
f\left(k^{T}\right)=\left(k^{T}\right)^{\theta^{T}}, \quad 0<\theta^{T}<1, \quad h\left(k^{N}\right)=\left(k^{N}\right)^{\theta^{N}}, \quad 0<\theta^{N}<1,
$$

where $\theta^{T}$ and $\theta^{N}$ represent the output share of capital income in the traded and non traded sector respectively.

We first briefly discuss the calibration. Based on empirical estimates provided by Cashin and McDermott [2003], we set the intertemporal elasticity of substitution $\sigma_{c}$ to 0.7 and the intratemporal elasticity of substitution $\phi$ to 2 implying that traded and non traded goods are substitutes in consumption (i. e. $c_{p}^{T}>0$ ). Following Stockman and Tesar [1995], the parameter $\varphi$ is assigned so as the the non tradable content in total consumption expenditure denoted by $\alpha_{c}$ falls in the range $40-60 \%$. Hence, $\varphi$ is set to 0.5 . A critical parameter is the elasticity of labor supply. Kimball and Shapiro [2003] provide an extensive discussion on empirical estimates of the compensated elasticity of labor supply. While empirical studies based on micro data find low values, say broadly lower than 0.5 , the real business cycle literature often set higher values for $\sigma_{L}$, say broadly equal or higher than unity. In our benchmark parametrization, we choose an average value and set $\sigma_{L}$ to 0.75 . The world interest rate, which is constrained to equalize the subjective time discount rate $\delta$, is chosen to be $4 \%$. The rate of depreciation of capital is set to $6 \%$.

We calculate the output shares of capital income, the markup, the tax rates, and tax allowances, which are summarized in Table 4 . The markup has been estimated by using the consistent methodology by Roeger [1995]; $\mu$ is set to 1.43 if $k^{T}>k^{N}$ and 1.49 if $k^{N}>k^{T}$, in line with empirical evidence; we calculated the effective tax rates on consumption and labor by using OECD database. Since the adjustment of a two-sector country depends on the relative sectoral capital intensities, we consider two different scenarios ; in the first scenario, 
Table 4: Benchmark parameter values

\begin{tabular}{lccl}
\hline Parameter & \multicolumn{2}{c}{ Value } & \\
& $k^{T}>k^{N}$ & $k^{N}>k^{T}$ & Definition \\
\hline$\sigma_{c}$ & 0.70 & 0.70 & Intertemporal elasticity of substitution \\
$\sigma_{L}$ & 0.75 & 0.75 & Frisch elasticity of labor supply \\
$\gamma$ & 0.90 & 0.90 & Scaling parameter in labor disutility function \\
$\varphi$ & 0.50 & 0.50 & Share of traded goods in consumer's bundle \\
$\phi$ & 2.00 & 2.00 & Intratemporal elasticity of substitution \\
$\beta$ & 0.04 & 0.04 & Subjective time discount rate \\
$\delta_{K}$ & 0.06 & 0.06 & Rate of physical capital depreciation \\
$\theta^{T}$ & 0.37 & 0.28 & Capital share in the Traded sector \\
$\theta^{N}$ & 0.31 & 0.33 & Capital share in the Non Traded sector \\
$\mu$ & 1.43 & 1.49 & Markup charged by the non traded sector \\
$\tau^{c}$ & 0.135 & 0.175 & Consumption tax rate \\
$\tau^{F}$ & 0.125 & 0.184 & Employer's payroll tax rate \\
$\tau^{H}$ & 0.257 & 0.354 & Employee's wage tax rate \\
$\kappa$ & 0.20 & 0.15 & Tax allowance \\
\hline
\end{tabular}

the traded sector is more capital intensive than the non traded sector $\left(\theta^{T}>\theta^{N}\right)$; in the second scenario, the non traded sector is more capital intensive than the traded sector $\left(\theta^{N}>\theta^{T}\right)$.

\section{A Labor Tax Cut}

As understated in Picard and Toulemonde [2003], in perfectly competitive markets, it does not matter to after-tax wage income and employment whether labor taxes are levied on employers or employees. A fall in the labor $\operatorname{tax} \tau^{j}(j=F, H)$ induces agents to supply more labor. As after-tax labor income increases, individuals are induced to raise their real expenditure. An excess demand arises in the non traded good market which leads to an investment boom and a current account deficit. While the rising after-tax labor income lowers unambiguously $\bar{\lambda}$ as long as $k^{T}>k^{N}$, the real exchange rate appreciation originating from the excess demand in the non traded goods market moderates the fall in $\bar{\lambda}$ by reducing the real wage and thus labor supply with the reversal of capital intensities.

\section{A Rise in Consumption Tax}

A rise in $\tau^{c}$ raises the marginal cost of consumption which in turn induces agents to reduce their real expenditure. An excess supply arises in the non traded good market which in turn requires a LT fall (resp. rise) in the capital stock if $k^{N}>k^{T}$ (resp. $k^{T}>k^{N}$ ). The decumulation of physical capital induces a LT rise in traded bonds holding. Since the government returns the net tax receipts to agents in a lump-sum transfer $\mathrm{d} Z>0$, households get richer such that $\bar{\lambda}$ falls. As long as $k^{N}>k^{T}$, a secondary channel plays by modifying the relative price of non tradables. The excess supply in the non traded good market induces an immediate depreciation in $p$ which in turn stimulates consumption and labor supply by reducing $p_{c}$ and raising $w$ respectively. Since the latter effect predominates, $\bar{\lambda}$ decreases further. 
To conclude, numerical results reported in Table 3 show that LT effects are almost similar across all 6 scenarios. Households lower or raise real consumption and labor supply by about $1 \%$ depending on wether the government raises the consumption tax rate by $3 \%$ or cuts the labor tax by the same amount. A rise in $\tau^{c}$ crowds-out capital accumulation and depresses output by less than $1 \%$. Unlike, a labor tax cut stimulates investment and overall output.

\section{Three Tax Reform strategies}

Since countries differ markedly in their tax structure, we consider three types of tax restructuring. More specifically, we explore two revenue-neutral tax reforms which involve simultaneously either cutting payroll taxes by $\mathrm{d} \tau^{F}<0$ or cutting labor income taxes by $\mathrm{d} \tau^{H}<0$ and raising the consumption tax rate by $\mathrm{d} \tau^{c}>0$. While the two previous tax reforms cause a fall in the marginal tax wedge, we study a third tax reform which involves simultaneously cutting payroll taxes by $\mathrm{d} \tau^{F}<0$ and raising labor income taxes by $\mathrm{d} \tau^{H}>0$ so as to leave the marginal tax wedge, denoted by $\tau^{M}$, constant.

\subsection{Long-Terms Effects of Revenue-Neutral Tax Reforms}

To avoid confusion, we denote by $\left.\right|^{j, c}$ the effects of a fall in the labor tax by $\mathrm{d} \tau^{j}<0(j=$ $F, H$ ) coordinated with a rise in the consumption tax rate by $\left.\mathrm{d} \tau^{c}\right|^{j, c}$ which is endogenously determined so as the government budget constraint is met.

\section{The Endogenous Rise in $\tau^{c}$}

Suppose that the policy maker wishes to replace the labor tax with a consumption tax, keeping the budget constraint balanced. For unchanged labor supply and consumption decisions, the labor tax cut (resp. increase in the consumption tax) leads to a fall (resp. a rise) in tax revenue commonly labelled the tax rate effect. In addition, a change of a distorsionary tax modifies the behavior of households. This induces a tax base effect which works in opposite direction of the tax rate effect on public revenue. Since the consumption tax rate is treated as an endogenous variable, the labor tax reform strategy requires a change of $\tau^{c}$ by an amount given by:

$$
\left.\mathrm{d} \tau^{c}\right|^{j, c}=-\frac{\chi^{j}}{\chi^{c}} \mathrm{~d} \tau^{j}=-\left\{\frac{\tau^{c} p_{c} \frac{\mathrm{d} \tilde{c}}{\mathrm{~d} \tau^{j}}+\left(\tilde{w}^{F}-\tilde{w}^{A}\right) \frac{\mathrm{d} \tilde{L}}{\mathrm{~d} \tau^{j}}+\Gamma^{j}}{\tau^{c} p_{c} \frac{\mathrm{d} \tilde{c}}{\mathrm{~d} \tau^{c}}+\left(\tilde{w}^{F}-\tilde{w}^{A}\right) \frac{\mathrm{d} \tilde{L}}{\mathrm{~d} \tau^{c}}+\Gamma^{c}}\right\} \mathrm{d} \tau^{j}>0,
$$

where we used (12) and $\chi^{c}>0$ and $\chi^{j} \gtrless 0$. We denoted by $\Gamma^{j}(j=F, H)$ and $\Gamma^{c}$ the labor and the consumption tax rate effects:

$$
\Gamma^{F}=\frac{1-\tau^{H}}{1+\tau^{F}} \tilde{w} \tilde{L}, \quad \Gamma^{H}=(w-\kappa) \tilde{L}, \quad \Gamma^{c}=p_{c} \tilde{c} .
$$

The terms $\chi^{j}$ and $\chi^{c}$ capture two conflictory effects induced by a reduction of the labor tax rate and an increase in the consumption tax rate respectively on public revenue. For given 
levels of real consumption and employment, a change of the tax rate modifies tax earnings as it is reflected by $\Gamma^{j}$ and $\Gamma^{c}$ (see the third terms on the RHS of (25)). However, as shown by the two first terms of the numerator and the denominator on the RHS of (25), the resulting LT adjustment of employment and consumption moderate the labor and the consumption tax rate effects. For the benchmark parametrization, $\chi^{c}$ and $\chi^{j}$ are positive which reflects the fact that influence of the tax rate effect on tax revenue more than that offsets the impact of the tax base effect. ${ }^{14}$ Consequently, $\tau^{c}$ must rise to compensate the tax revenue loss induced by the labor tax cut.

To have a sense of the magnitude of the labor and consumption tax base effect which counteract the tax rate effects, we estimated the following ratios:

$$
\frac{\chi^{j}}{\Gamma^{j}} \simeq 0.68-0.79 \text { and } \frac{\chi^{c}}{\Gamma^{c}} \simeq 0.81-0.85 \text { and } \frac{\Gamma^{H}}{\Gamma^{c}} \simeq 0.77, \quad \frac{\Gamma^{F}}{\Gamma^{c}} \simeq 0.52-0.64
$$

depending on wether case $k^{N} \gtrless k^{T}$. These ratios show to what extent does a tax cut pay for itself. For example, the numerical values of $\frac{\chi^{j}}{\Gamma^{j}}$ and $\frac{\chi^{c}}{\Gamma^{c}}$ which are approximately 0.72 and 0.83 respectively show that a fall by $1 \%$ of public revenue induced by a labor tax rate shrinks to $0.72 \%$ due to the labor tax base effect (i. e. the resulting LT increases in consumption and labor) and a rise in $1 \%$ in public revenue induced by the increase in the consumption tax rate shrinks to $0.83 \%$ due to the consumption tax base effect (i. e. the resulting LT decreases in consumption and labor).

The magnitude of the rise in $\tau^{c}$ can be spilt into two components: the extent to which the tax base effects compensate the tax rate effects and the ratio labor tax base-consumption tax base, i. e. $\frac{\frac{\chi^{j}}{\Gamma^{j}}}{\frac{\chi^{c}}{\Gamma^{c}}} \frac{\Gamma^{j}}{\Gamma^{c}}$. While the first component plays a minor role, the ratio $\frac{\Gamma^{F}}{\Gamma^{c}}$ is primarily responsible of $\left.\mathrm{d} \tau^{c}\right|^{j, c}$. The second component is approximately equal to 0.58 which means that the labor tax base is about two-times smaller than the consumption tax base. Hence, a labor tax cut by 1 percentage point requires a rise in $\tau^{c}$ which is in average approximately halfway of the labor tax cut.

\section{Steady-State Changes}

We now analyze the overall effects of a shift of the tax burden from labor taxes to consumption taxes. After some manipulations, the LT change of $x$ following a shift in the tax burden is given by: ${ }^{15}$

$$
\left.\mathrm{d} \tilde{x}\right|^{j, c}=\frac{\mathrm{d} \tilde{x}}{\mathrm{~d} \tau^{j}} \mathrm{~d} \tau^{j}+\left.\frac{\mathrm{d} \tilde{x}}{\mathrm{~d} \tau^{c}} \mathrm{~d} \tau^{c}\right|^{j, c} \equiv \Phi^{j, c} \frac{\mathrm{d} \tilde{x}}{\mathrm{~d} \tau^{j}} \mathrm{~d} \tau^{j}>0,
$$

where $\Phi^{j, c}=\left[1-\frac{\tilde{w}^{A} \tilde{L}}{p_{c}\left(1+\tau^{c}\right) \tilde{c}}\right]$ for $j=F, H$. A sufficient condition for $\Phi^{j, c}$ to be positive but smaller than unity is that households are net creditor, i. e. $\tilde{a}>0 .{ }^{16}$ The overall outcome a tax restructuring is equal to the sum of the LT rise in $x$ after a labor tax cut by $\mathrm{d} \tau^{j}<0$ and the steady-state fall in $x$ following a rise in by $\left.\mathrm{d} \tau^{c}\right|^{j, c}>0$. Interestingly, according to (27), the steady-state change of $x$ following a tax restructuring is a scaled-down version of the LT changes of $x=c, L, K$ after a fall in the labor $\operatorname{tax} \tau^{j}(j=F, H)$ financed by lump-sum taxes. The larger the share of financial wealth in real disposable income, the larger the consumption 
tax base in comparison to the labor tax base, and thereby the less $\tau^{c}$ must increase for a given labor tax cut so as the government budget constraint to be met. Consequently, the tax restructuring gets more effective as the scaled-down term $\Phi^{j, c}$ gets closer to unity. This result stems from the fact that in the neoclassical model, a reduction of labor taxes financed by a rise in a distorsionary tax have smaller effects than a tax cut financed by lump-sump tax financing. For our benchmark parametrization, the scaled-down $\Phi^{j, c}$ falls in a range between 0.36 and 0.51 depending on wether $k^{T} \gtrless k^{N}$; additionally, $\Phi^{j, c}$ gets closer to unity as the consumption tax base gets larger compared to the labor tax base. Since the steady-state change of $x$ following a tax restructuring is a scaled-down version of the LT effect after a cut in $\tau^{j}$ financed by a rise in lump-sum taxes, a second condition for a steady-state increase in $x$ is that $d x / \tau^{j}>0$.

Table 5: Quantitative Effects of Three Tax Reforms

\begin{tabular}{|c|c|c|c|c|c|c|}
\hline \multirow[b]{2}{*}{ Tax Reform } & \multicolumn{3}{|c|}{$k^{T}>k^{N}$} & \multicolumn{3}{|c|}{$k^{N}>k^{T}$} \\
\hline & $\mathrm{d} \tau^{F}$ & $\mathrm{~d} \tau^{H}$ & $\mathrm{~d} \tau^{F}$ & $\mathrm{~d} \tau^{F}$ & $\mathrm{~d} \tau^{H}$ & $\mathrm{~d} \tau^{F}$ \\
\hline Financed by & $\mathrm{d} \tau^{c}$ & $\mathrm{~d} \tau^{c}$ & $\mathrm{~d} \tau^{H}$ & $\mathrm{~d} \tau^{c}$ & $\mathrm{~d} \tau^{c}$ & $\mathrm{~d} \tau^{H}$ \\
\hline \multicolumn{7}{|c|}{ A. Tax rates changes } \\
\hline$\overline{\mathrm{d} \tilde{\tau}^{c}}$ & 1.8 & 2.1 & & 1.3 & 1.9 & \\
\hline $\mathrm{d} \tilde{\tau}^{F}$ & -3.0 & & -3.0 & -3.0 & & -3.0 \\
\hline $\mathrm{d} \tilde{\tau}^{H}$ & & -3.0 & 2.0 & & -3.0 & 1.6 \\
\hline \multicolumn{7}{|l|}{ B.Long-Term } \\
\hline $\mathrm{d} \tilde{Y}$ & 0.32 & 0.37 & 0.19 & 0.40 & 0.58 & 0.15 \\
\hline $\mathrm{d} \tilde{L}$ & 0.31 & 0.36 & 0.18 & 0.41 & 0.59 & 0.15 \\
\hline $\mathrm{d} \tilde{c}$ & 0.34 & 0.40 & 0.20 & 0.46 & 0.66 & 0.17 \\
\hline $\mathrm{d} \tilde{K}$ & 0.33 & 0.38 & 0.19 & 0.39 & 0.56 & 0.14 \\
\hline \multicolumn{7}{|c|}{ C.Short-Term } \\
\hline $\mathrm{d} L(0) / \tilde{L}_{0}$ & 0.31 & 0.36 & 0.18 & 0.27 & 0.47 & 0.10 \\
\hline $\mathrm{d} c(0) / \tilde{c}_{0}$ & 0.34 & 0.40 & 0.20 & 0.43 & 0.61 & 0.16 \\
\hline $\mathrm{d} I(0) / \tilde{Y}_{0}$ & 1.21 & 1.40 & 0.71 & 1.50 & 2.15 & 0.55 \\
\hline $\mathrm{d} c a(0) / \tilde{Y}_{0}$ & -1.21 & -1.40 & -0.71 & -1.72 & -2.46 & -0.62 \\
\hline $\mathrm{d} S(0) / \tilde{Y}_{0}$ & 0 & 0 & 0 & -0.22 & -0.32 & -0.08 \\
\hline
\end{tabular}

Whereas a rise in government spending crowds-out private consumption and stimulates labor supply, a tax restructuring crowds-in consumption and encourages labor supply in the LT. To have a better grasp of our result, it is convenient to split the steady-state changes into a wealth effect and a tax effect:

$$
\left.\mathrm{d} \tilde{c}\right|^{j, c}=\left.m_{\bar{\lambda}} \mathrm{d} \bar{\lambda}\right|^{j, c}+\left.m_{\tau^{c}} \mathrm{~d} \tau^{c}\right|^{j, c}>0,\left.\quad \mathrm{~d} \tilde{L}\right|^{j, c}=\left.n_{\bar{\lambda}} \mathrm{d} \bar{\lambda}\right|^{j, c}+n_{\tau^{j}} \mathrm{~d} \tau^{j}>0, \quad j=F, H,
$$

where $m_{\bar{\lambda}}<0, m_{\tau^{c}}<0, n_{\bar{\lambda}}>0, n_{\tau^{j}}<0(j=F, H)$; it can be shown formally that tax reform strategy pushes down the marginal utility of wealth, irrespective of sectoral capital intensities. Households are induced to raise both consumption and labor can be easily understood from inspection of (28). The tax effect which impinges negatively on the LT value of real consumption displays a smaller size than its positive influence on labor since $\tau^{c}$ must increase 
but by less than the size of the labor tax cut. In line with theoretical results, as shown by row 2 in Table 5B, the steady-state rise in real consumption gets larger as the required rise in the consumption tax rate gets smaller. For example, a payroll tax cut by $3 \%$ leads to an increase in $\tau^{c}$ by $1.3 \%$ (resp. $1.8 \%$ ) if $k^{N}>k^{T}$ (resp. $k^{T}>k^{N}$ ) and $\tilde{c}$ is raised by $0.46 \%$ (resp. 0.34\%). While the steady-state increase in real consumption stems from the wealth effect, the LT rise in labor supply originates from the tax effect. The smaller the fall in the marginal utility of wealth and the larger the steady-state increase in $L$.

The LT change of the physical capital stock can be more easily discussed by differentiating totally the market-clearing condition for non tradables: ${ }^{17}$

$$
\left.\frac{1}{\mu}\left(Y_{K}^{N}-\delta_{K}\right) \mathrm{d} \tilde{K}\right|^{j, c}+\left.\frac{1}{\mu} Y_{L}^{N} \mathrm{~d} \tilde{L}\right|^{j, c}=\left.\mathrm{d} \tilde{c}^{N}\right|^{j, c}>0, \quad j=F, H,
$$

where $Y_{K}^{N}-\delta_{K} \gtrless 0, Y_{L}^{N} \lessgtr 0, Y_{\tau^{j}}^{N} \gtrless 0(j=F, H)$ depending on wether $k^{N} \gtrless k^{T}$. While it can be shown formally the capital stock rises in the LT, the mechanism behind this result requires to consider two cases. If $k^{N}>k^{T}$ (resp. $k^{T}>k^{N}$ ), the labor outflow (resp. inflow) from (resp. in) the more capital intensive sector reduces (resp. stimulates) $Y^{N}$ while the demand for the non traded good rises. Consequently, an excess demand (resp. excess supply) arises which requires a $\mathrm{LT}$ rise in the the capital stock. In any cases, the tax reform leads to an investment boom which depresses the output of the relatively more labor intensive sector. As the open economy accumulates physical capital $K$ over the transition towards the steady-state, agents reduce their foreign assets holding $\tilde{b}$. For the current account to be null in the LT, net exports $\tilde{n x}$ must rise to compensate the decrease in interest receipts from traded bonds holding.

As it can be seen from Table 5B, in line with theoretical results, a tax restructuring boosts capital accumulation in the LT. Depending on wether employees or employers benefit from the labor tax cut, the steady-state rise in the overall capital stock falls in the range between $0.38 \%-0.56 \%$ if $\tau^{H}$ is decreased and $0.33 \%-0.39 \%$ if $\tau^{F}$ is cut. While for our benchmark parametrization, the former tax policy displays stronger effects on capital accumulation regardless of sectoral capital-labor ratios, we show in the next section that this result depends on the initial marginal tax wedge and the degree of tax progressiveness. The relevancy of this discussion is supported by the cross-country comparison of the these two variables which display sizeable differences.

\section{A Cut in the Labor Income Tax or in the Payroll Tax?}

In this section, we provide an analytical comparison of the steady-state effects of a tax restructuring depending on wether the labor tax cut falls on the labor income tax or the payroll tax, i. e.; depending on wether employees or employers benefit from the reduction in $\tau^{j}$. A shift of the tax burden from labor taxes to consumption taxes implies that the LT effect of a tax restructuring is a scaled-down version of the LT change after a cut in $\tau^{j}$ financed by lump-sum taxes. Irrespective of the labor tax cut benefit to employers or employees, the scaled-down positive factor $\Phi^{j, c}$ is identical. The two tax reforms differ mainly in that the

LT change of $x$ after a fall in $\tau^{H}$ is a scaled-down or a scaled-up version of the steady-state change of $x$ after a drop in $\tau^{F}$. To see it more formally, we express the steady-state change 
of $x$ after a cut in $\tau^{H}$ in terms of the LT variation of $x$ following a reduction of $\tau^{F}$ :

$$
\frac{\mathrm{d} \tilde{x}}{\mathrm{~d} \tau^{H}}=\frac{\tilde{w}-\kappa}{\tilde{w}} \frac{1+\tau^{F}}{1-\tau^{H}} \frac{\mathrm{d} \tilde{x}}{\mathrm{~d} \tau^{F}}
$$

Technically, the size of the positive term $\frac{\tilde{w}-\kappa}{\tilde{w}} \frac{1+\tau^{F}}{1-\tau^{H}}$, which might be higher or smaller than unity, depends on the initial tax wedge $\tau^{M}$ and the degree of tax progressiveness. Intuitively, a cut in $\tau^{F}$ raises the wage rate and thereby the after-tax labor income by $\tilde{w} \frac{1-\tau^{H}}{1+\tau^{F}} \equiv \tilde{w}\left(1-\tau^{M}\right)$. Rather, a drop in $\tau^{H}$ leaves unaffected $w$ and raises the after-tax labor income by $(\tilde{w}-\kappa)$. In brief, a tax restructuring displays stronger positive effects on labor market if households benefit from the labor tax cut than if payroll taxes are reduced as longer as tax allowances $\kappa$ gets smaller and the initial marginal tax wedge $\tau^{M}$ gets closer to unity.

Finally, an interesting feature is that the effectiveness of a tax restructuring decreases with the degree of tax progressiveness, i. e. as tax allowances $\kappa$ rises, since the taxable labor income $w-\kappa$ and thereby the incentive to raise labor supply gets smaller. In brief, the tax progressiveness moderates the rise of the fall in the after-tax labor income and consequently the LT effects of a tax restructuring which involves a cut in $\tau^{H}$.

\subsection{Dynamics Effects of Consumption and Labor Tax Reforms}

Having discussed the steady-state changes, which influence in turn agents' expectations because of the perfect foresight property, we now investigate the dynamic effects of a shift of the tax burden from labor taxes (i. e. a cut in $\tau^{j}, j=F, H$ ) to consumption taxes (i. e. a rise in $\tau^{c}$ ).

If $k^{T}>k^{N}$, the dynamics of the relative price of non tradables degenerate so that labor and real consumption increase immediately to their final LT levels. Instead, if $k^{N}>k^{T}$, the temporal path of the real exchange rate is no longer flat which in turns restores the transitional dynamics for consumption and labor. Figures 1 portray the computed transitional paths for the real exchange rate, consumption, labor, investment, savings and the current account after a tax restructuring involving simultaneously cutting $\tau^{H}$ and raising $\tau^{c}{ }^{18}$ The increase in the after-tax labor income induces a positive wealth effect which boosts consumption as shown in Figures 1(c) and 1(a). As demand for non tradables rises and labor shifts towards the more labor intensive sector, an excess demand arises in the non traded good market. This excess demand appreciates the real exchange rate on impact by $0.05 \%$ (see Table $5 \mathrm{C}$ ); more formally, $\left.\mathrm{d} p(0)\right|^{j, c}=-\left.\omega_{2}^{1} \mathrm{~d} \tilde{K}\right|^{j, c}>0$. The rise in the relative price of non tradables softens the initial upward jumps of real consumption and labor supply by raising the consumption price index and reducing the real wage:

$$
\begin{aligned}
\left.\mathrm{d} c(0)\right|^{j, c} & =\left.\mathrm{d} \tilde{c}\right|^{j, c}+\left.c_{p} \mathrm{~d} p(0)\right|^{j, c}>0, \quad j=F, H, \\
\left.\mathrm{~d} L(0)\right|^{j, c} & =\left.\mathrm{d} \tilde{L}\right|^{j, c}+\left.L_{p} \mathrm{~d} p(0)\right|^{j, c}>0, \quad j=F, H,
\end{aligned}
$$

where $c_{p}<0$ and $L_{p}<0$ (if $k^{N}>k^{T}$ ). The rise in private wealth induces agents to raise consumption by about $0.6 \%$ and increase labor supply by $0.5 \%$. 


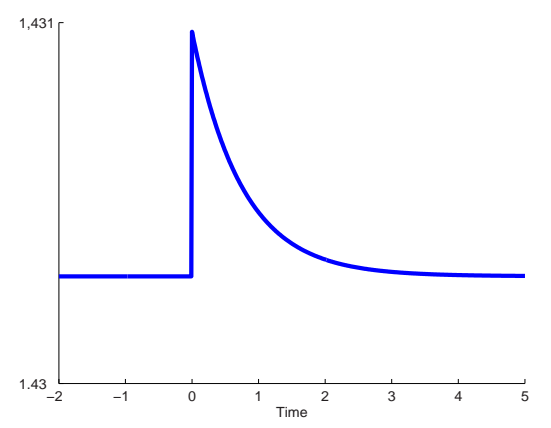

(a) Transitional Dynamics for $\mathrm{p}(\mathrm{t})$

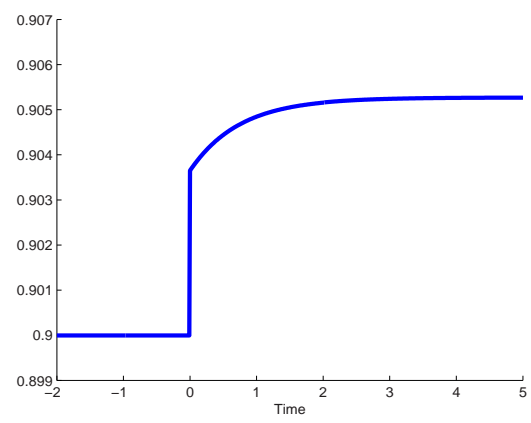

(c) Transitional Dynamics for L(t)

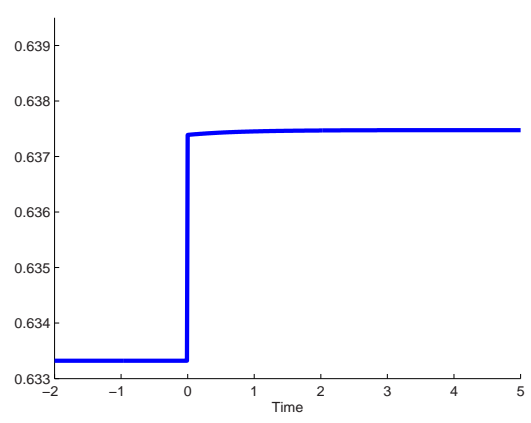

(b) Transitional Dynamics for $\mathrm{c}(\mathrm{t})$

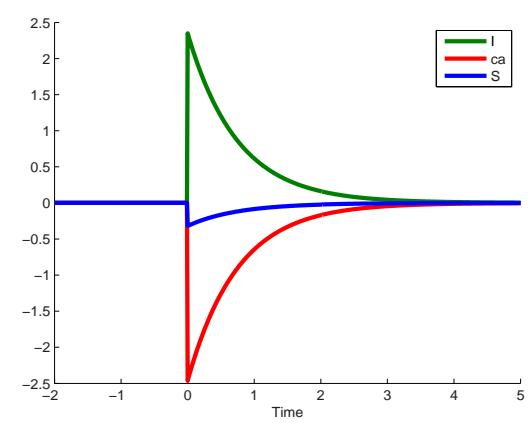

(d) Transitional Dynamics for $\mathrm{I}(\mathrm{t}), \mathrm{S}(\mathrm{t}), \mathrm{ca}(\mathrm{t})$

Figure 1: Computed Transitional Paths after a Fall in $\tau^{H}$ and a Rise in $\left.\tau^{c}\right|^{H, c}$ - Case $k^{N}>k^{T}$

The real exchange appreciation attracts resources in the non traded sector if $k^{N}>k^{T}$ which in turn leads to an investment boom on impact:

$$
\left.\mathrm{d} I(0)\right|^{j, c}=\left.Y_{L}^{N} \mathrm{~d} L(0)\right|^{j, c}-\left.\hat{Y}_{p}^{N} \mathrm{~d} p(0)\right|^{j, c}-\left.\mathrm{d} c^{N}(0)\right|^{j, c}>0
$$

where we used the fact that the capital stock is initially given, i. e. $K(0)=K_{0}$ and we denoted by the superscript hat the derivative of $Y^{N}$ with respect to $p$ for given employment (set $\sigma_{L}=0$ ). If $k^{T}>k^{N}$, the influence of the real exchange vanishes; the increase in total employment induces a shift of labor away from the traded sector towards the non traded sector; this leads to a positive investment flow even whereas the rise in consumption in non tradables withdraws resources to capital accumulation. Interestingly, as shown in Table 5C, a tax reform crowds-in investment by more if $k^{N}>k^{T}$ than with the reversal of capital intensities (i e. $1.5 \%$ and $2.1 \%$ rather than $1.2 \%$ and $2.4 \%$ ). The explanation is that the real exchange rate appreciation on impact attracts resources in the non traded sector and thereby stimulates capital accumulation further in the ST .

Since capital accumulation is crowded-in further by a tax restructuring in the case $k^{N}>k^{T}$ and agents decumulate financial wealth, the open country experiences a larger current account deficit as shown by row 4 in Table $5 \mathrm{C}$ (i e. $-1.7 \%$ and $-2.5 \%$ rather than $-1.2 \%$ and $-1.4 \%$ ).

Along the transitional path, the real exchange rate must depreciate to revert back to its initial level. By increasing the consumption-based real interest rate and the real wage, the real exchange rate depreciation induces a rising temporal paths for consumption and labor which in turn drives progressively down the size of the investment flow and consequently the 
size of the current account deficit. If $k^{T}>k^{N}$, savings is unaffected because the positive investment flow is exactly matched by a current account deficit.

\subsection{A Labor Tax Restructuring}

In this section, we explore the effects of a labor tax reform strategy denoted by the superscript $\{F, H\}$ which involves simultaneously cutting a payroll tax by $\mathrm{d} \tau^{F}<0$ and increasing the labor income tax $\mathrm{d} \tau^{H}>0$ so as to leave unaffected the marginal tax wedge (i. e. $\mathrm{d} \tau^{M}=0$ ). The labor tax reform strategy requires a rise in the wage income tax by an amount given by:

$$
\left.\mathrm{d} \tau^{H}\right|^{F, H} \equiv-\theta \mathrm{d} \tau^{F}, \quad \theta \equiv \frac{1-\tau^{H}}{1+\tau^{F}}<1 .
$$

From (33), the labor income tax must be increased by a smaller amount than the fall in $\tau^{F}$ for leaving unchanged the marginal tax wedge. In short, since the tax rate on a relatively large base is reduced and the tax rate on a relatively small base is increased, the latter must rise by a smaller proportion than the former decreases so as to leave unchanged $\tau^{M}$.

The LT change of $x=c, L, K$ following a cut in $\tau^{F}$ coordinated with a rise in $\tau^{H}$ by an amount given by (33) writes as follows: ${ }^{19}$

$$
\left.\mathrm{d} \tilde{x}\right|^{F, H}=\Phi^{F, H} \frac{\mathrm{d} \tilde{x}}{\mathrm{~d} \tau^{F}}=\frac{\kappa}{\tilde{w}} \mathrm{~d} \tau^{F},
$$

where $\Phi^{F, H} \equiv \kappa / \tilde{w}<1$. As longer as the labor income tax rate is progressive, i. e. $\kappa>0$, then the labor tax reform leaving constant the marginal tax wedge affects the LT equilibrium of the economy. More specifically, the steady-state increases in consumption, employment and capital stock are a scaled-down version of their LT changes following a labor tax cut financed by lump-sum taxes.

Interestingly, tax progression benefits employment and overall economic activity because the wage rate unambiguously rises as it can be shown formally:

$$
\left.\mathrm{d} w^{A}\right|^{F, H}=-\kappa\left(\frac{1-\tau^{H}}{1+\tau^{F}}\right) \mathrm{d} \tau^{F}>0
$$

where we assume that $\mathrm{d} \tau^{F}<0$. As the wage rate is permanently raised and labor supply is mostly determined by the tax effect, employment rises in the LT. More specifically, as the degree of tax progression rises, as reflected by an increase in tax allowances $\kappa$, the rise in the after-tax labor income gets larger which in turn magnifies the beneficial effects on employment and overall economic activity. ${ }^{20}$ Instead, if $\kappa$ is set to zero, then a larger rise in $\tau^{H}$ is required so that the LT equilibrium of the open economy remains unchanged.

What is the effect of the reform on public revenue? The overall effect of a labor tax restructuring so as to leave unaffected $\tau^{M}$ induces two conflictory effects on public revenue. Without tax base effects, a negative revenue effect would arise. Intuitively, the tax rate on a relatively large tax base is lowered while the tax rate on a relatively small base is raised. However, via the tax effect for employment and the wealth effect for consumption, the tax bases increase but not sufficiently as as to offset the negative tax rate effect. Accordingly, lump-sum transfers drop by $-3.39 \%$ if $k^{T}>k^{N}$ and $-0.85 \%$ if $k^{N}>k^{T}$. 


\subsection{Short-Term and Long-Term Tax Multipliers}

Having discussed the steady-state and dynamics effects, we now investigate the effectiveness of the three tax reform strategies by calculating the LT and ST tax multipliers for output at an overall and a sectoral level.

\section{The Overall Tax Multiplier}

Using the fact that in the LT, overall output equalizes its demand counterpart, differentiating by adopting a similar procedure than we used previously, and keeping in mind that the steady-state level of the relative price of non tradables is not affected by a tax restructuring, the LT tax multiplier for overall output is given by: ${ }^{21}$

$$
\left.\mathrm{d} \tilde{Y}\right|^{j, k}=\left.p_{c} \mathrm{~d} \tilde{c}\right|^{j, k}+\left.\mathrm{d} \tilde{n x}\right|^{j, k}+\left.\tilde{p} \mathrm{~d} \tilde{I}\right|^{j, k}>0,
$$

where we denoted net exports by $n x \equiv Y^{T}-\left(c^{T}+g^{T}\right)$. According to (36), the the domestic demand boom for consumption and investment goods is strengthened by a trade balance improvement. Since the tax reform raises overall demand, aggregate output unambiguously rises in the LT. Table 6 reports the numerical values of LT tax multipliers for overall output. For the benchmark parametrization, the LT multipliers are positive and fall in the range between 0.06-0.25. As expected, a revenue-neutral tax reform displays larger beneficial effects than a strategy keeping the tax wedge constant since in the latter case the demand boom is strongly moderated by the fall in lump-sump transfers.

We now derive the ST change of overall output by linearizing overall demand for the domestic good, i. e. $Y=p_{c} c+\left(g^{T}+p g^{N}\right)+p(t) I(t)+n x(t)$, evaluating at time $t=0$ and differentiating the resulting linearized version of this expression: ${ }^{22}$

$$
\left.\mathrm{d} Y(0)\right|^{j, k}=\left.p_{c} \mathrm{~d} c(0)\right|^{j, k}+\left.\tilde{p} \mathrm{~d} I(0)\right|^{j, k}+\left.\tilde{Y}^{N} \mathrm{~d} p(0)\right|^{j, k}+\left.\mathrm{d} n x(0)\right|^{j, k}>0 .
$$

The change of overall output on impact is driven by the initial demand boom for consumption goods and investment goods together with the strong real exchange rate appreciation which raises the value of non traded output when expressed in terms of the traded good. While in the LT, the trade balance improvement raises further GDP, the fall in net exports on impact, which reflects the current account deficit, lowers the effectiveness of the tax reform.

Let now investigate wether a tax reform displays more beneficial effects in the ST or in the LT. While the ST change of output benefits from the real exchange rate appreciation, the steady-state rise in net exports raises the steady-state value of GDP above that in the ST:

$$
0<\left.\mathrm{d} Y(0)\right|^{j, k}<\left.\mathrm{d} \tilde{Y}\right|^{j, k}
$$

The numerical values reported in the Table 6 show that the tax multiplier is $1 / 3$ or $50 \%$ smaller in the ST than in the LT depending on wether the traded sector is more or less capital intensive than the non traded sector. The smaller size of the ST tax multiplier stems from the fact that part of the additional labor income is devoted to imports on impact which reduces as much as the demand for the domestic good. In addition, the ST tax multiplier is reduced further 
Table 6: LT and ST Tax Multipliers for Overall Output

\begin{tabular}{|c|c|c|c|c|c|c|}
\hline \multirow[b]{2}{*}{ Tax Reform } & \multicolumn{3}{|c|}{$k^{T}>k^{N}$} & \multicolumn{3}{|c|}{$k^{N}>k^{T}$} \\
\hline & $\mathrm{d} \tau^{F}$ & $\mathrm{~d} \tau^{H}$ & $\mathrm{~d} \tau^{F}$ & $\mathrm{~d} \tau^{F}$ & $\mathrm{~d} \tau^{H}$ & $\mathrm{~d} \tau^{F}$ \\
\hline Financed by & $\mathrm{d} \tau^{c}$ & $\mathrm{~d} \tau^{c}$ & $\mathrm{~d} \tau^{H}$ & $\mathrm{~d} \tau^{c}$ & $\mathrm{~d} \tau^{c}$ & $\mathrm{~d} \tau^{H}$ \\
\hline Long-Term & 0.16 & 0.18 & 0.09 & 0.16 & 0.25 & 0.06 \\
\hline Short-term & 0.10 & 0.14 & 0.06 & 0.08 & 0.13 & 0.03 \\
\hline
\end{tabular}

since the strong real exchange rate appreciation on impact raises the consumption price index which thereby depresses private demand.

\section{The Sectoral Tax Multiplier}

So far, we investigated the effectiveness at the level of the overall economy. One central question in a two-sector model is: Which sector will benefit by more the tax reform strategy? We now show formally that regardless of sectoral capital intensities or the kind of tax reform, the tax multipliers in the traded and the non traded sector are both positive which implies that a sector does not expand at the expense of the other sector.

Table 7: LT and ST Tax Multipliers for $Y^{T}$ and $Y^{N}$

\begin{tabular}{|c|c|c|c|c|c|c|}
\hline \multirow[b]{2}{*}{ Tax Reform } & \multicolumn{3}{|c|}{$k^{T}>k^{N}$} & \multicolumn{3}{|c|}{$k^{N}>k^{T}$} \\
\hline & $\mathrm{d} \tau^{F}$ & $\mathrm{~d} \tau^{H}$ & $\mathrm{~d} \tau^{F}$ & $\mathrm{~d} \tau^{F}$ & $\mathrm{~d} \tau^{H}$ & $\mathrm{~d} \tau^{F}$ \\
\hline Financed by & $\mathrm{d} \tau^{c}$ & $\mathrm{~d} \tau^{c}$ & $\mathrm{~d} \tau^{H}$ & $\mathrm{~d} \tau^{c}$ & $\mathrm{~d} \tau^{c}$ & $\mathrm{~d} \tau^{H}$ \\
\hline \multicolumn{7}{|l|}{ Long-Term } \\
\hline Sector $T$ & 0.09 & 0.10 & 0.05 & 0.09 & 0.13 & 0.03 \\
\hline Sector $N$ & 0.07 & 0.08 & 0.04 & 0.08 & 0.11 & 0.03 \\
\hline \multicolumn{7}{|l|}{ Short-Term } \\
\hline Sector $T$ & -0.53 & -0.62 & -0.31 & -0.63 & -0.91 & -0.23 \\
\hline Sector $N$ & 0.64 & 0.74 & 0.37 & 0.71 & 1.02 & 0.26 \\
\hline
\end{tabular}

Using the market-clearing condition together with the null current account equation to derive the LT expenditure multipliers in the non traded and traded sector respectively:

$$
\begin{aligned}
\left.\frac{1}{\mu} \mathrm{d} \tilde{Y}^{N}\right|^{j, k} & =\left.\mathrm{d} \tilde{c}^{N}\right|^{j, k}+\left.\mathrm{d} \tilde{I}\right|^{j, k}>0, \\
\left.\mathrm{~d} \tilde{Y}^{T}\right|^{j, k} & =\left.\mathrm{d} \tilde{n x}\right|^{j, k}+\left.\mathrm{d} \tilde{c}^{T}\right|^{j, k}>0 .
\end{aligned}
$$

Since a tax restructuring induces a positive wealth effect by raising the after-tax labor income, the demand for both the traded and the non traded consumption goods unambiguously rises. Additionally, while the traded sector benefits from the trade balance improvement, the LT stimulus of capital investment boosts the non traded output. Consequently tax multipliers are positive in both sectors. It is worthwhile noticing that in the LT, the economic boom in sector $\mathrm{N}$ enhances the traded output. The explanation is that the LT capital accumulation 
drives the current account into deficit which requires a steady-state improvement in the trade balance. The numerical values reported in Table 7 show that the steady-state improvement in the trade balance raises the LT tax multiplier in sector $\mathrm{T}$ above the LT tax multiplier in sector N. However, the sectoral discrepancy is modest. In conclusion, while the tradable share in overall output is about $1 / 3$, its contribution to the growth of GDP amounts to about one half.

Interestingly, we get a different picture in the ST:

$$
\begin{aligned}
\left.\frac{1}{\mu} \mathrm{d} Y^{N}(0)\right|^{j, k} & =\left.\mathrm{d} c^{N}(0)\right|^{j, k}+\left.\mathrm{d} I(0)\right|^{j, k}>0, \\
\left.\mathrm{~d} Y^{T}(0)\right|^{j, k} & =\left.\mathrm{d} n x(0)\right|^{j, k}+\left.\mathrm{d} c^{T}(0)\right|^{j, k} \gtrless 0 .
\end{aligned}
$$

From (40a), output in sector $\mathrm{N}$ is unambiguously stimulated by the domestic demand boom for consumption and investment goods $I$. Unlike, in the traded sector, the dramatic drop in net exports on impact now counteracts the positive influence of the domestic demand boom for the traded good. If $k^{T}>k^{N}$, it can be proven formally that the tax multiplier in the traded sector is negative on impact which reflects the fact that the trade balance deficit more than outweighs the domestic demand boom. With the reversal of capital intensities, we cannot exclude that the short-tern tax multiplier is positive in the traded sector. Numerical values of ST sectoral tax multipliers summarized in Table 7 show that output in sector $\mathrm{T}$ drops on impact while the economic boom in sector $\mathrm{N}$ is sizeable. Considerable discrepancy in the sectoral economic stance stemming from the $I$ boom and the trade balance deficit in the ST.

\subsection{Welfare Effects}

Since the ultimate objective of individuals is their satisfaction, it is appropriate to study the welfare effects of a tax reform. We start by the investigation of instantaneous welfare dynamics which is denoted by $\xi \equiv u(c(t))+v(L(t))$. Linearizing the felicity function in the neighborhood of the steady-state, then substituting the formal solution for $p$ given by (19), we get the stable path for $\xi(t)$.

Welfare effects may be most easily interpreted by contrasting the LT and ST reactions:

$$
\left.\mathrm{d} \tilde{\phi}\right|^{j, k}=\Phi^{j, k}\left[u_{c} \frac{\mathrm{d} \tilde{c}}{\mathrm{~d} \tau^{j}}+v_{L} \frac{\mathrm{d} \tilde{L}}{\mathrm{~d} \tau^{j}}\right] \mathrm{d} \tau^{j},\left.\quad \mathrm{~d} \phi(0)\right|^{j, k}=\left.\mathrm{d} \tilde{\phi}\right|^{j, k}+\left.\Xi \mathrm{d} \tilde{K}\right|^{j, k}
$$

where $0<\Phi^{j, k}<1$ and $\left.\mathrm{d} \tilde{K}\right|^{j, k}>0$; the term $\Xi$ is positive for the benchmark parametrization. Whereas households have welfare gains from higher consumption, they are subject to utility losses triggered by labor supply. If $k^{T}>k^{N}$, the former predominates over the latter such that $\phi$ unambiguously rises in the LT. Instead, if $k^{N}>k^{T}$, the households' wealth is affected permanently by the transitional adjustment of the relative price of non tradables which raises further labor supply and moderates the LT rise in real consumption.

In order to have an appealing measure of welfare, we estimate a measure of overall welfare 
Table 8: Overall Welfare Effects of a Tax Reform

\begin{tabular}{|c|c|c|c|c|c|c|}
\hline \multirow[b]{2}{*}{ Tax Reform } & \multicolumn{3}{|c|}{$k^{T}>k^{N}$} & \multicolumn{3}{|c|}{$k^{N}>k^{T}$} \\
\hline & $\mathrm{d} \tau^{F}$ & $\mathrm{~d} \tau^{H}$ & $\mathrm{~d} \tau^{F}$ & $\mathrm{~d} \tau^{F}$ & $\mathrm{~d} \tau^{H}$ & $\mathrm{~d} \tau^{F}$ \\
\hline Financed by & $\mathrm{d} \tau^{c}$ & $\mathrm{~d} \tau^{c}$ & $\mathrm{~d} \tau^{H}$ & $\mathrm{~d} \tau^{c}$ & $\mathrm{~d} \tau^{c}$ & $\mathrm{~d} \tau^{H}$ \\
\hline Overall Welfare & 0.05 & 0.06 & 0.03 & 0.09 & 0.12 & 0.03 \\
\hline
\end{tabular}

which is defined by the discounted value of felicity flows over an infinite horizon:

$$
U=\int_{0}^{\infty} \xi(t) e^{-\beta t} \mathrm{~d} t
$$

Substituting the stable solution for $\phi(t)$ and integrating, evaluating the resulting expression at time $t=0$ and differentiating, we get:

$$
\left.\mathrm{d} U\right|^{j, k}=\left.\frac{1}{\beta} \mathrm{d} \tilde{\xi}\right|^{j, k}+\frac{\mathrm{d}\left(\left.\xi(0)\right|^{j, k}-\left.\tilde{\xi}\right|^{j, k}\right)}{r^{\star}-\nu_{1}} .
$$

The LT rise in $\xi$ raises overall welfare while its transitional adjustment (which raises further $U$ ) reflected by the second term on the RHS of (43) exerts a small influence. The computed overall welfare gains are summarized in Table 8. Numerical results show that a tax restructuring always raises households' stance in utility terms which fall in the range between $0.03 \%$ and $0.12 \%$. Besides the effectiveness gains it induces, a tax reform yields welfare improvement.

\section{$5 \quad$ Sensitivity Analysis}

One important contribution of our analysis is to shed light on the roles of two critical parameters in determining the size of the tax multiplier: [i] the elasticity of labor supply $\sigma_{L}$ and [ii] the degree of tax progressiveness $\Gamma$.

\section{Tax Multipliers}

Figures 2(a)-2(b) portray the LT and ST overall tax multipliers against $\sigma_{L}$. Like Baxter et King [1993], the LT tax multiplier rises with $\sigma_{L}$. As labor gets more responsive to the rise in the after-tax labor income, the excess of demand (resp. supply) in the non traded good market gets larger if $k^{N}>k^{T}$ (resp. if $k^{T}>k^{N}$ ), the physical capital investment must be crowded-in further to clear the good market, and thereby the LT multiplier gets larger. However, unlike Baxter and King [1993], the relationship between the ST tax multiplier and the elasticity labor supply displays a hump-shaped pattern. As labor supply gets more responsive, the excess of demand in the non traded good market gets larger, the real exchange appreciates by more on impact which in turn softens further the ST demand boom for consumption goods. Along the slippery slope side, the depressing effect on domestic demand triggered by the impact appreciation in $p$ more than offsets the stimulating effect induced by the rise in the after-tax labor income. 


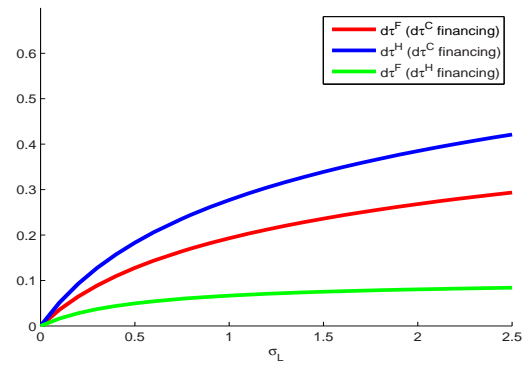

(a) Plot of LR tax multipliers against $\sigma_{L}$

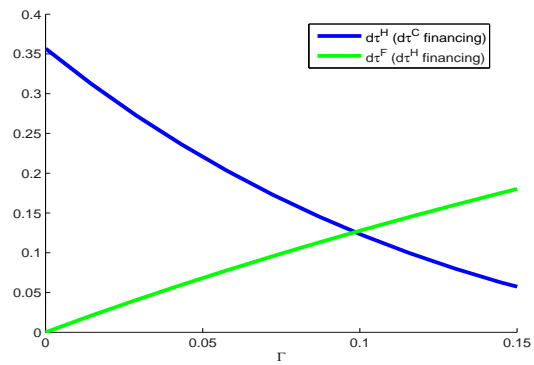

(c) Plot of LR Tax Multipliers against Tax Progressiveness

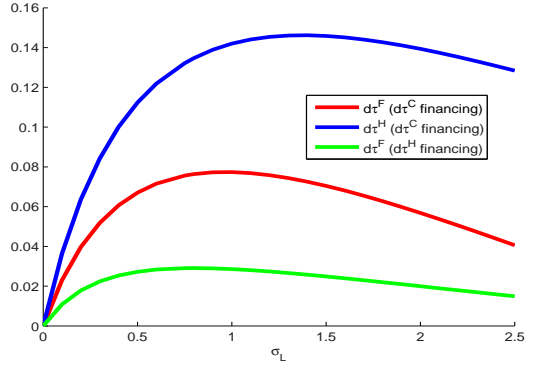

(b) Plot of SR tax multipliers against $\sigma_{L}$

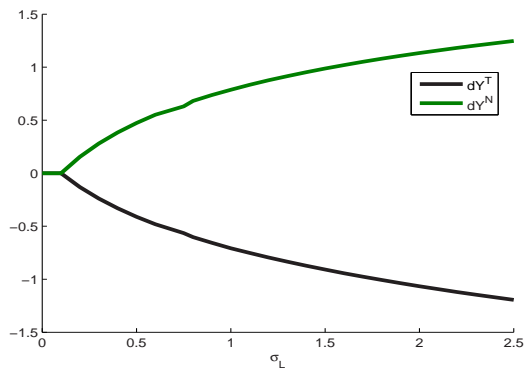

(d) Plot of SR Sectoral Tax Multipliers against $\sigma_{L}$

Figure 2: Sensitivity of the Overall and Sectoral Tax Multipliers to the Degree of Tax Progressiveness and $\sigma_{L}$

Figure 2(c) traces out the LT tax multiplier against the degree of tax progressiveness. According to our measure, the tax scheme gets more progressive as the indicator $\Gamma$ gets larger. Ceteris paribus, an increase in tax allowances $\kappa$ at a given wage rate yields an increase in average tax progression. Figure 2(c) shows that the LT tax multiplier falls or rises with the degree of tax progressiveness depending on wether the tax reform is revenue neutral or keeps the tax wedge constant. In the latter case, the depressing effect triggered by higher labor income tax rate gets smaller as the tax scheme gets more progressive. Instead, in the former case, labor rises by less since the after-tax labor income increases by a smaller amount.

Finally, we get a more complete and interesting picture of a tax reform by conducting a sensitivity analysis at a sectoral level. The figure 2(d) traces out the ST sectoral tax multiplier against the elasticity of labor supply in the case $k^{N}>k^{T}$ and for a tax reform strategy which moves the tax burden from payroll taxes to consumption taxes. Interestingly, the ST tax multiplier in the non traded sector rises and in the traded sector falls as labor supply gets more responsive. The explanation is as follows. As labor supply displays more reactiveness to the rise in the after-tax labor income, the employment outflow (and thereby the excess demand in the non traded good market) gets larger. Consequently, the tax reform crowds-in investment by more which in turn drives the current account (and hence the trade balance) into a larger deficit. In short, while the initial boom for capital goods boosts the non traded output, the dramatic drop in net exports on impact depressed the traded output. 


\section{Conclusion}

We employ a two-sector open economy version of the dynamic general equilibrium model by Baxter and King [1993] to examine the potential benefits of three tax restructuring. Based on empirical evidence, we distinguish between a traded and a non traded sector and assume the non tradables are produced by an imperfectly competitive sector. In line with general practice, we assume that wage taxes are progressive whereas payroll taxes are supposed to be proportional. We consider two budget-neutral strategies that shifts the payroll or personal labor income taxes to consumption taxes and one strategy keeping the marginal tax wedge constant that reduces the taxes paid by employers and raises the taxes paid by employees.

Irrespective of sectoral capital intensities, consumption and investment are unambiguously crowded-in and employment is permanently raised, as long as the consumption tax base is larger than the labor tax base. While the steady-state increase in consumption is driven by a positive wealth effect, the steady-state rise in employment is driven by the higher after-tax labor wage induced by the labor tax cut. The second finding is that the shift of the tax burden from labor taxes to consumption taxes so as to keep the tax revenue fixed is more effective than a strategy keeping the tax wedge constant. While estimating the effectiveness of the tax reform by calculating the tax multiplier, numerical results show that the long-term tax multiplier rises or falls with the degree of tax progressiveness depending on wether the tax reform keeps the tax wedge constant or keeps the government budget balanced.

Regardless of the type of tax reform, the sensitivity analysis shows that the long-term tax multiplier for overall output rises monotonically with elasticity of labor supply. Unlike, the relationship between the short-term tax and labor responsiveness displays a hump-shaped pattern stemming from the depressing effect on domestic demand triggered by the real exchange rate appreciation. One major question in a two-sector country is: Which sector will benefit by more from the tax reform? The answer to this question highlights the central role of the trade balance. Since net exports must rise in the long-term to exactly offset the fall in net interest earnings from foreign assets holding, the long-term tax multiplier is always higher in the traded sector than in the non-traded sector. However, the computed sectoral discrepancy is not considerable. Interestingly, the result is reversed in the short-term. In the traded sector, the dramatic drop in net exports on impact now counteracts the positive influence of the domestic demand boom for the traded good. Numerical results show a considerable discrepancy in the sectoral economic stance stemming from the investment boom which boosts the non traded sector output and the trade balance deficit which depresses the traded output.

\section{Notes}

\footnotetext{
${ }^{1}$ Another branch of the literature, exemplified by the work of Mankiw and Weinzierl [2006] and Leeper and Yang [2008], focuses on capital and labor tax cuts and estimates to what extent reductions in capital and labor income tax rates expand the tax base to offset revenue losses.
} 
${ }^{2}$ While the two-revenue neutral tax reforms lowers the marginal tax wedge constant, we consider a third strategy which involves simultaneously cutting payroll taxes and raising labor income taxes so as to keep the tax wedge constant. Whereas this labor tax restructuring does no longer keep the tax revenue fixed, it allows us to focus on the composition on the tax wedge rather than its level.

${ }^{3}$ The labor income tax rate encompasses the social security contributions paid by employees together with personal income taxes.

${ }^{4}$ We restrict the countries panel table to the biggest OECD countries. A more complete picture is presented is an Appendix available by the author on request.

${ }^{5}$ This particular subutility function (1) because it yields a constant price elasticity demand equations for each variety. We follow Startz [1989] in specifying the leading term in the subutility function, $n^{\frac{1}{1-\sigma}} ;$ this formulation is adopted for reason of analytical simplicity since it eliminates the "taste of variety" inherent in the usual CES form. Hence, individuals only care about the overall amount $c^{N}$ which is consumed and thus adopt a neutral attitude towards variety.

${ }^{6}$ See Greenwood et al. [1988] who specify a similar form for $v(L)$.

${ }^{7}$ Overall fixed costs $n F C$ are thus covered by setting the price above the marginal cost, i. e. by paying inputs below their marginal products.

${ }^{8}$ To obtain static solutions for traded and non traded output, we first solve (13e) and (13f) for $L^{T}$ and $L^{N}$. We get $L^{T}=L^{T}\left(K, p, \bar{\lambda}, \tau^{F}, \tau^{H}\right), L^{N}=L^{N}\left(K, p, \bar{\lambda}, \tau^{F}, \tau^{H}\right)$ with $L_{K}^{T} \lessgtr 0, L_{\bar{\lambda}}^{T} \gtrless 0, L_{K}^{N} \gtrless 0, L_{\bar{\lambda}}^{N} \lessgtr 0, L_{\tau^{j}}^{T} \lessgtr 0$ and $L_{\tau^{j}}^{N} \gtrless 0$ (with $j=H, F$ ) according to wether $k^{N} \gtrless k^{T}$, together with $L_{p}^{T}<0$ and $L_{p}^{N}>0$.

${ }^{9}$ To determine the accumulation equation of foreign bonds (13j), we substituted the definition of transfers $Z$ together with the non traded goods market-clearing condition (13i) into (6) and used the fact that $\left(r^{K}+\delta_{K}\right) K^{N}+w^{F} L^{N} \equiv \frac{p}{\mu} Y^{N}$ and $r^{K} K^{T}+w^{F} L^{T} \equiv Y^{T}$.

${ }^{10}$ If $k^{N}>k^{T}$, the sign of $\Phi_{1}=-\tilde{p}\left[1+\frac{\omega_{2}^{1}}{\tilde{p} \mu_{2}}\left(\sigma_{c} \tilde{c}^{N}-\sigma_{L} \tilde{L} \tilde{k}^{T}\left(\mu_{2}+\delta_{K}\right) \tilde{\Lambda}\right)\right]$ is not clear-cut. Since for a large range of parametrization, $\Phi_{1}$ is always negative, we assume that capital accumulation deteriorates the current account.

${ }^{11}$ Intuitively, if $k^{N}>k^{T}$, an increase in demand for the non traded good causes a real exchange appreciation which shifts labor in sector $N$ and thereby stimulates capital accumulation. The combined effect of the negative savings flow and the positive investment flow drives the current account into deficit over entire transition towards the LT equilibrium.

${ }^{12}$ In the second step, we insert (23) into the economy's intertemporal budget constraint (i. e. equation $(22 \mathrm{~d})$ ), which may be solved for the equilibrium value of the marginal utility of wealth: $\bar{\lambda}=g\left(\tau^{c}, \tau^{F}, \tau^{H}\right)$, with $\lambda_{\tau^{c}}<0, \lambda_{\tau^{F}}>0, \lambda_{\tau^{H}}>0$.

${ }^{13}$ If $k^{N}>k^{T}$, the sign of $K_{\bar{\lambda}}$ is not clear-cut. However, for a large range of parametrization, numerical results show that $\sigma_{L} \tilde{L} \tilde{k}^{T} \mu_{2} \tilde{\Lambda}-\sigma_{c} \tilde{c}^{N}>0$ which reflects the fact that the labor response is stronger than that of consumption to the real exchange rate depreciation over the transitional adjustment.

${ }^{14}$ While it can be proven formally that the consumption tax rate effect is large enough to more than offsets the consumption tax base effect as longer as $\tilde{a}>0$, such a condition cannot longer be derived for determining the sign of $\chi^{j}$.

${ }^{15}$ The derivation of (27) requires to rewrite first the steady-state variation of $x$ after a rise in $\tau^{c}$ in terms of that after a change of $\tau^{j}$ financed by lump-sum taxes: $\frac{\mathrm{d} \tilde{x}}{\mathrm{~d} \tau^{c}}=\frac{1}{1+\tau^{c}} \frac{\mathrm{d} \tilde{x}}{\mathrm{~d} \tau^{j}} \Xi^{j}$, with $\Xi^{F}=\frac{1+\tau^{F}}{\tilde{\Lambda}}>0$ and $\Xi^{H}=\frac{\tilde{w}^{A}}{\tilde{w}-\kappa}>0$. Formal details can be retrieved in Appendix F.

${ }^{16}$ More specifically, using the steady-state version of the budget constraint of households, we can express the 
share in real disposable income of interest earnings from assets holding plus lump-sum transfers as follows:

$$
\frac{r^{\star} \tilde{a}+Z}{p_{c} \tilde{c}}=1-\frac{\tilde{w}^{A} \tilde{L}}{p_{c} \tilde{c}},
$$

A sufficient condition for the expression to be positive is that $\tilde{a}>0$.

${ }^{17}$ We found convenient to rewrite at the steady-state the ST static solutions for traded and non traded output given by $(18)$ as $\tilde{Y}^{T}=Y^{T}(\tilde{K}, \tilde{L}, \tilde{p})$ and $\tilde{Y}^{N}=Y^{N}(\tilde{K}, \tilde{L}, \tilde{p})$.

${ }^{18}$ Since computed transitional paths are similar for either three tax reforms, the dynamics portrayed in Figure 1 of the two-sector economy is restricted to a cut in the labor income tax coordinated with a rise in the consumption tax that keeps the government budget constraint balanced.

${ }^{19}$ To derive (34), we used the fact that $\frac{\mathrm{d} \tilde{x}}{\mathrm{~d} \tau^{H}}=\frac{(\tilde{w}-\kappa)\left(1+\tau^{F}\right)}{\tilde{w}\left(1-\tau^{H}\right)} \frac{\mathrm{d} \tilde{x}}{\partial \tau^{F}}$.

${ }^{20}$ Both the steady-state and the dynamic effects are similar to that derived in sections 4.1 and 4.2 ; hence we do not discuss them further.

${ }^{21}$ Using the market-clearing condition, i. e. $\frac{\tilde{Y}^{N}}{\mu}=\tilde{c}^{N}+g^{N}+\tilde{I}$, and the null current account, i. e. $\tilde{Y}^{T}=$ $\left(\tilde{c}^{T}+g^{T}\right)-r^{\star} \tilde{b}$, aggregating and differentiating, we get (36).

${ }^{22}$ Using the fact that $Y \equiv Y^{T}+\frac{p}{\mu} Y^{N}$, and keeping in mind that the capital stock is initially predetermined, the ST tax multiplier can be written as follows:

$$
\left.\mathrm{d} Y(0)\right|^{j, k}=\left.w^{F} \mathrm{~d} L(0)\right|^{j, k}>0 .
$$

Since labor rises on impact as the after-tax labor income increases, the ST tax multiplier is unambiguously positive, irrespective of sectoral capital intensities and regardless of the kind of the tax reform strategy.

\section{References}

Baxter, Marianne and Robert G. King (1993) Fiscal Policy in General Equilibrium. American Economic Review, 83(3), pp. 315-334.

Cardi, Olivier (2007) The Zero-Root Property: Permanent Vs Temporary Terms-of-Trade Shocks. Review of International Economics, 15(4), pp. 782-802. 306-325.

Cashin, Paul and John C. McDermott (2003) Intertemporal Substituion and Terms-ofTrade Shocks. Review of International Economics, vol. 11 (4), pp. 604-618.

Coenen, Günter, Peter McAdam and Roland Straub (2008) Tax Reform and LabourMarket Performance in the Euro Area: A Simulation-Based Analysis Using the New AreaWide Model. Journal of Economic Dynamics and Control, forthcoming.

Coto-Martinez, Javier and Huw Dixon (2003) Profits, Markups and Entry: Fiscal Policy in an Open Economy. Journal of Economic Dynamics and Control, 27(4), pp. 573-597.

De Gregorio, J., Giovannini A. and Wolf H.C. (1994) International Evidence on Tradables and Nontradables Inflation. European Economic Review 38, pp. 1225-1244.

Ganelli, Giovanni, and Juha Tervala (2008) Tax Reforms, "Free Lunches", and "Cheap Lunches" in Open Economies. IMF Working Paper $n^{\circ} 227$. 
Greenwood, Jeremy, Zvi Hercovitz, and Gregory W. Huffman (1988) Investment, Capacity Utilization, and the Real Business Cycle. American Economic Review, 78(3), 402-417.

Heijdra, Ben J., and Jenny E. Ligthart (2008) Labor Tax Reform, Unemployment and Search. International Tax and Public Finance, forthcoming.

Kimball, Miles and Shapiro, Matthew (2003) Labor Supply: Are the Income and Substitution Effects Both Large or Both Small. Unpublished, University of Michigan.

Leeper, Eric M., and Shu-Chun Susan Yang (2008) Dynamic Scoring: Alternative Financing Schemes. Journal of Public Economics 92, pp. 159-182.

Lin, Shouyong (1999) Tax Reform and External Balance. Journal of International Money and Finance 18, pp. 891-909.

Mankiw, Gregory N., and Weinzierl Matthew (2006) Dynamic Scoring: A Back-of-theEnvelope Guide. Journal of Public Economics 88(1), pp. 226-245.

Mendoza, Enrique G., and Linda Tesar (1998) The International Ramifications of Tax Reforms: Supply-Side Economics ina Global Economy. American Economic Review, 81(4), 797-818.

Morshed Mahbub A. K. M., and Stefen J. Turnovsky (2004) Sectoral Adjustment Costs and Real Exchange Rate Dynamics in a Two-Sector Dependent Economy. Journal of International Economics 63, 147-177.

Newey, Whitney and Kenneth West (1987) A Simple Positive Semi-Definite, Heteroskedasticity and Autocorrelation Consistent Covariance Matrix, Econometrica, Vol. (51), pp. 703-708.

Obstfeld, Maurice (1989) Fiscal Deficits and Relative Prices in a Growing World Economy. Journal of Monetary Economics 23, 461-484.

Picard, Pierre M. and Eric Toulemonde (2003) Taxation and Labor Markets. Journal of Economics, 78, pp. 29-56.

Roeger, Werner (1995) Can Imperfect Competition Explain the Difference between Primal and Dual Productivity Measures? Estimates for U.S. Manufacturing. The Journal of Political Economy 103(2), pp. 316-330.

Startz, Richard (1989) Monopolistic Competition as a Foundation For Keynesian Macroeconomic Models. Quarterly Journal of Economics, 104(4), pp. 737-752.

Stockman Alan C. and Tesar Linda L. (1995) Tastes and Technology in a Two-Country Model of the Business Cycle: Explaining International Comovements. American Economic Review, 85(1), pp. 168-185. 
Trabandt, Mathias, and Harald Uhlig (2006) How Far are we from the Slippery Slope? The Laffer Curve Revisited. Mimeo. Humboldt University Berlin.

Turnovsky, Stephen J. and Partha Sen (1995) Investment in a Two-Sector Dependent Economy. Journal of the Japenese and International Economies 9, pp. 29-55. 


\section{TAX REFORM IN TWO-SECTOR GENERAL EQUILIBRIUM}

TECHNICAL APPENDIX

OCTOBER 2008

Olivier CARDI AND Romain RESTOUT 


\section{A Short-Run Static Solutions}

\section{A.1 Short-Run Static Solutions for Consumption-Side}

Short-run static solutions for real consumption and labor supply are derived from the firstorder conditions (13a) and (13b):

$$
c=c\left(\bar{\lambda}, p, \tau^{c}\right), \quad L=L\left(\bar{\lambda}, p, \tau^{F}, \tau^{H}\right),
$$

with

$$
\begin{aligned}
c_{\bar{\lambda}} & =\frac{\partial c}{\partial \bar{\lambda}}=-\sigma_{c} \frac{c}{\bar{\lambda}}<0, \\
c_{p} & =\frac{\partial c}{\partial p}=-\alpha_{c} \sigma_{c} \frac{c}{p}<0, \\
c_{\tau^{c}} & =\frac{\partial c}{\partial \tau^{c}}=-\sigma_{c} \frac{c}{\left(1+\tau^{c}\right)}<0, \\
L_{\bar{\lambda}} & =\frac{\partial L}{\partial \bar{\lambda}}=\sigma_{L} \frac{L}{\bar{\lambda}}>0, \\
L_{p} & =\frac{\partial L}{\partial p}=\sigma_{L} L \frac{w_{p}\left(1-\tau^{H}\right)}{w^{A}}=-\sigma_{L} L \frac{\Lambda}{w^{F}} \frac{k^{T} h}{\mu\left(k^{N}-k^{T}\right)} \lessgtr 0, \\
L_{\tau^{F}} & =\frac{\partial L}{\partial \tau^{F}}=-\sigma_{L} L \frac{w_{\tau^{F}}\left(1-\tau^{H}\right)}{w^{A}}=-\sigma_{L} L \frac{\Lambda}{\left(1+\tau^{F}\right)}<0, \\
L_{\tau^{H}} & =\frac{\partial L}{\partial \tau^{H}}=-\sigma_{L} L \frac{(w-\kappa)}{w^{A}}<0,
\end{aligned}
$$

where $\sigma_{c}=-\frac{u_{c}}{u_{c c} c}>0$ corresponds to the intertemporal elasticity of substitution for consumption, $\sigma_{L}=\frac{v_{L}}{v_{L L} L}>0$ represents the intertemporal elasticity for labor. We denoted by $0<\Lambda \equiv \frac{\left(1-\tau^{H}\right)}{\left[\left(1-\tau^{H}\right)+\frac{\tau_{\kappa}}{w}\right]}<1$ as longer as $\kappa>0$; if $\kappa=0$, then $\Lambda=1$. According to (45f), labor supply decreases or increases following a real exchange rate appreciation depending on wether $k^{N} \gtrless k^{T}$.

Denoting by $\phi$ the intratemporal elasticity of substitution between the tradable and the non tradable good and inserting short-run solution for consumption (44) into intra-temporal allocations between non tradable and tradable goods, we solve for $c^{T}$ and $c^{N}$ :

$$
c^{T}=c^{T}\left(\bar{\lambda}, p, \tau^{c}\right), \quad c^{N}=c^{N}\left(\bar{\lambda}, p, \tau^{c}\right),
$$


with

$$
\begin{aligned}
c_{\bar{\lambda}}^{T} & =-\sigma_{c} \frac{c^{T}}{\bar{\lambda}}<0, \\
c_{p}^{T} & =\alpha_{c} \frac{c^{T}}{p}\left(\phi-\sigma_{c}\right) \lessgtr 0, \\
c_{\tau^{c}}^{T} & =-\sigma_{c} \frac{c^{T}}{\left(1+\tau^{c}\right)}<0, \\
c_{\bar{\lambda}}^{N} & =-\sigma_{c} \frac{c^{N}}{\bar{\lambda}}<0, \\
c_{p}^{N} & =-\frac{c^{N}}{p}\left[\left(1-\alpha_{c}\right) \phi+\alpha_{c} \sigma_{c}\right]<0, \\
c_{\tau^{c}}^{N} & =-\sigma_{c} \frac{c^{N}}{\left(1+\tau^{c}\right)}<0,
\end{aligned}
$$

where we used the fact that $-\frac{p_{c}^{\prime \prime} p}{p_{c}^{\prime}}=\phi\left(1-\alpha_{c}\right)>0$ and $p_{c}^{\prime} c=c^{N}$.

\section{A.2 Short-Run Static Solutions for Production-Side}

\section{Capital-Labor Ratios}

From static optimality conditions (13c) and (13d), we may express sector capital-labor ratios as functions of the real exchange rate:

$$
k^{T}=k^{T}(p), \quad k^{N}=k^{N}(p),
$$

with

$$
\begin{aligned}
& k_{p}^{T}=\frac{\partial k^{T}}{\partial p}=\frac{h}{\mu f_{k k}\left(k^{N}-k^{T}\right)}, \\
& k_{p}^{N}=\frac{\partial k^{N}}{\partial p}=\frac{\mu f}{p^{2} h_{k k}\left(k^{N}-k^{T}\right)} .
\end{aligned}
$$

\section{Wage}

From the first-order condition (13d), we can solve for the wage rate:

$$
w=w\left(p, \tau^{F}\right),
$$

with

$$
\begin{aligned}
w_{p} & =\frac{\partial w}{\partial p}=-\frac{k^{T} f_{k k} k_{p}^{T}}{\left(1+\tau^{F}\right)}=-\frac{k^{T}}{\left(1+\tau^{F}\right)} \frac{h}{\mu\left(k^{N}-k^{T}\right)} \lessgtr 0, \\
w_{\tau^{F}} & =\frac{\partial w}{\partial \tau^{F}}=-\frac{w}{\left(1+\tau^{F}\right)}<0 .
\end{aligned}
$$

\section{Labor}

Substituting short-run static solutions for labor (44) and capital-labor ratios (48) into sectoral inputs allocation constraints (13e) and (13f), we can solve for traded and non traded labor as follows:

$$
L^{T}=L^{T}\left(K, p, \bar{\lambda}, \tau^{F}, \tau^{H}\right), \quad L^{N}=L^{N}\left(K, p, \bar{\lambda}, \tau^{F}, \tau^{H}\right),
$$


with

$$
\begin{aligned}
L_{K}^{T} & =\frac{\partial L^{T}}{\partial K}=\frac{1}{k^{T}-k^{N}} \lessgtr 0, \\
L_{p}^{T} & =\frac{\partial L^{T}}{\partial p}=\frac{1}{\mu\left(k^{N}-k^{T}\right)^{2}}\left[\frac{L^{T} h}{f_{k k}}+\frac{\mu^{2} L^{N} f}{p^{2} h_{k k}}-\sigma_{L} L \frac{\Lambda}{w^{F}} k^{T} k^{N} h\right]<0, \\
L_{\bar{\lambda}}^{T} & =\frac{\partial L^{T}}{\partial \bar{\lambda}}=\sigma_{L} \frac{L}{\bar{\lambda}} \frac{k^{N}}{k^{N}-k^{T}} \gtrless 0, \\
L_{\tau^{F}}^{T} & =\frac{\partial L^{T}}{\partial \tau^{F}}=-\frac{k^{N}}{k^{N}-k^{T}} \sigma_{L} L \frac{\Lambda}{\left(1+\tau^{F}\right)} \lessgtr 0, \\
L_{\tau^{H}}^{T} & =\frac{\partial L^{T}}{\partial \tau^{H}}=-\frac{k^{N}}{k^{N}-k^{T}} \sigma_{L} L \frac{(w-\kappa)}{w^{A}} \lessgtr 0, \\
L_{K}^{N} & =\frac{\partial L^{N}}{\partial K}=\frac{1}{k^{N}-k^{T}} \gtrless 0, \\
L_{p}^{N} & =\frac{\partial L^{N}}{\partial p}=-\frac{1}{\mu\left(k^{N}-k^{T}\right)^{2}}\left[\frac{L^{T} h}{f_{k k}}+\frac{\mu^{2} L^{N} f}{p^{2} h_{k k}}-\sigma_{L} L \frac{\Lambda}{w^{F}}\left(k^{T}\right)^{2} h\right]>0, \\
L_{\bar{\lambda}}^{N} & =\frac{\partial L^{N}}{\partial \bar{\lambda}}=-\sigma_{L} \frac{L}{\bar{\lambda}} \frac{k^{T}}{k^{N}-k^{T}} \lessgtr 0, \\
L_{\tau^{F}}^{N} & =\frac{\partial L^{T}}{\partial \tau^{F}}=\frac{k^{T}}{k^{N}-k^{T}} \sigma_{L} L \frac{\Lambda}{\left(1+\tau^{F}\right)} \gtrless 0, \\
L_{\tau^{H}}^{N} & =\frac{\partial L^{N}}{\partial \tau^{H}}=\frac{k^{T}}{k^{N}-k^{T}} \sigma_{L} L \frac{(w-\kappa)}{w^{A}} \gtrless 0,
\end{aligned}
$$

where $w^{F}=w\left(1+\tau^{F}\right)$. From (53a) and (53f), when the capital stock rises, labor must shift to the sector which is relatively more capital intensive. From (53b) and (53g), a rise in the relative price of non tradable goods (lowers the capital-labor ratios) causes a shift of labor from the traded to the non-traded sector, irrespective of the sectoral capital intensities. From (53c) and (53h), an increase in the marginal utility of wealth raises the labor supplied by households which leads to a shift of labor to the sector which is relatively less capital capital intensive. From (53d)-(53e) and (53i)-(53j), a rise in the tax rate $\tau^{F}$ paid by firms or an increase in the tax rate $\tau^{H}$ paid by households reduces unambiguously total employment $L$ which leads to a shift of labor towards the sector which is relatively more capital intensive.

\section{Output}

Inserting short-run static solutions for capital-labor ratios (48) and for labor (53) into the production functions, we can solve for the traded, $Y^{T}=L^{T} k^{T}$, and the non traded output, $Y^{N}=L^{N} h^{N}$ :

$$
Y^{T}=Y^{T}\left(K, p, \bar{\lambda}, \tau^{F}, \tau^{H}\right), \quad Y^{N}=Y^{N}\left(K, p, \bar{\lambda}, \tau^{F}, \tau^{H}\right),
$$


with

$$
\begin{aligned}
Y_{K}^{T} & =\frac{\partial Y^{T}}{\partial K}=-\frac{f}{k^{N}-k^{T}} \lessgtr 0 \\
Y_{p}^{T} & =\frac{\partial Y^{T}}{\partial p}=\frac{1}{\mu\left(k^{N}-k^{T}\right)^{2}}\left[\frac{p L^{T}(h)^{2}}{\mu f_{k k}}+\frac{L^{N}(\mu f)^{2}}{(p)^{2} h_{k k}}-\sigma_{L} L \frac{\Lambda}{w^{F}} k^{T} k^{N} h f\right]<0, \\
Y_{\bar{\lambda}}^{T} & =\frac{\partial Y^{T}}{\partial \bar{\lambda}}=\sigma_{L} \frac{L}{\bar{\lambda}} \frac{k^{N} f}{k^{N}-k^{T}} \gtrless 0 \\
Y_{\tau^{F}}^{T} & =\frac{\partial Y^{T}}{\partial \tau^{F}}=-\frac{k^{N} f}{k^{N}-k^{T}} \sigma_{L} L \frac{\Lambda}{\left(1+\tau^{F}\right)} \lessgtr 0, \\
Y_{\tau^{H}}^{T} & =\frac{\partial Y^{T}}{\partial \tau^{H}}=-\frac{k^{N} f}{k^{N}-k^{T}} \sigma_{L} L \frac{(w-\kappa)}{w^{A}} \lessgtr 0, \\
Y_{K}^{N} & =\frac{\partial Y^{N}}{\partial K}=\frac{h}{k^{N}-k^{T}} \gtrless 0, \\
Y_{p}^{N} & =\frac{\partial Y^{N}}{\partial p}=-\frac{1}{p\left(k^{N}-k^{T}\right)^{2}}\left[\frac{p L^{T}(h)^{2}}{\mu f_{k k}}+\frac{L^{N}(\mu f)^{2}}{p^{2} h_{k k}}-\frac{p}{\mu} \sigma_{L} L \frac{\Lambda}{w^{F}}\left(k^{T} h\right)^{2}\right]>0 .(55) \\
Y_{\bar{\lambda}}^{N} & =\frac{\partial Y^{N}}{\partial \bar{\lambda}}=-\sigma_{L} \frac{L}{\bar{\lambda}} \frac{k^{T} h}{k^{N}-k^{T}} \lessgtr 0, \\
Y_{\tau^{F}}^{N} & =\frac{\partial Y^{N}}{\partial \tau^{F}}=\frac{k^{T} h}{k^{N}-k^{T}} \sigma_{L} L \frac{\Lambda}{\left(1+\tau^{F}\right)} \gtrless 0, \\
Y_{\tau^{H}}^{N} & =\frac{\partial Y^{N}}{\partial \tau^{H}}=\frac{k^{T} h}{k^{N}-k^{T}} \sigma_{L} L \frac{(w-\kappa)}{w^{A}} \gtrless 0 .
\end{aligned}
$$

From (55b) and (55g), an appreciation in the real exchange rate attracts resources from the traded to the non traded sector which in turn raises the output of the latter. From (55a) and (55f), a rise in the capital stock raises the output of the sector which is relatively more capital intensive. From (55c) and (55h), an increase in the marginal utility of wealth lowers the output in the sector which is relatively less capital intensive. From (55d)-(55e) and (55i)$(55 \mathrm{j})$, higher wage taxes, whatever they are levied on employers or employees depress total employment and favor the output in the sector which is relatively more capital intensive. As it will be useful to calculate fiscal multipliers, we give the partial derivatives of output in the traded and the non traded sector w. r. t. total employment:

$$
Y_{L}^{T}=\frac{\partial Y^{T}}{\partial L}=\frac{k^{N} f}{k^{N}-k^{T}} \gtrless 0, \quad Y_{L}^{N}=\frac{\partial Y^{N}}{\partial L}=-\frac{k^{T} h}{k^{N}-k^{T}} \lessgtr 0 .
$$

\section{Useful Properties}


Making use of (55b) and (55g), (55a) and (55f), we deduce the following useful properties:

$$
\begin{aligned}
Y_{p}^{T}+p \frac{Y_{p}^{N}}{\mu} & =-\sigma_{L} L \Lambda \frac{k^{T} h}{\mu\left(k^{N}-k^{T}\right)} \lessgtr 0, \\
\mu Y_{K}^{T}+p Y_{K}^{N} & =\frac{\mu f-p h}{k^{T}-k^{N}}=p h_{k}=\mu f_{k}, \\
Y_{L}^{T}+p \frac{Y_{L}^{N}}{\mu} & =w^{F}, \\
Y_{\bar{\lambda}}^{T}+p \frac{Y_{\bar{\lambda}}^{N}}{\mu} & =\sigma_{L} \frac{L}{\bar{\lambda}} \frac{\left(k^{N} \mu f-k^{T} p h\right)}{\mu\left(k^{N}-k^{T}\right)}=\sigma_{L} \frac{L}{\bar{\lambda}} w^{F}>0, \\
Y_{\tau^{F}}^{T}+p \frac{Y_{\tau^{F}}^{N}}{\mu} & =-\sigma_{L} L w \Lambda<0, \\
Y_{\tau^{H}}^{T}+p \frac{Y_{\tau^{H}}^{N}}{\mu} & =-\sigma_{L} L \frac{(w-\kappa)}{w^{A}} w^{F}<0,
\end{aligned}
$$

where we used the fact that $\mu f \equiv p\left[h-h_{k}\left(k^{N}-k^{T}\right)\right]$ and $k^{N} \mu f-k^{T} p h=$ $p\left(h-h^{K} k^{N}\right)\left(k^{N}-k^{T}\right)=\mu w^{F}\left(k^{N}-k^{T}\right)$.

In addition, using the fact that $r^{K}=f_{k}\left[k^{T}(p, \mu)\right]$, the rental rate of capital denoted by $r^{K}$ can be expressed as a function of the real exchange rate $p$ and the mark-up $\mu$ :

$$
r^{K}=r^{K}(p),
$$

with partial derivatives given by:

$$
r_{p}^{K} \equiv \frac{\partial r^{K}}{\partial p}=\frac{h}{\mu\left(k^{N}-k^{T}\right)} \gtrless 0 .
$$

\section{B Equilibrium Dynamics and Formal Solutions}

Inserting short-run static solutions (44), (46) and (54) into (13h) and (13i), we obtain:

$$
\begin{aligned}
\dot{K} & =\frac{1}{\mu} Y^{N}\left(K, p, \bar{\lambda}, \tau^{F}, \tau^{H}\right)-c^{N}\left(\bar{\lambda}, p, \tau^{c}\right)-\delta_{K} K-g^{N}, \\
\dot{p} & =p\left[r^{\star}-\frac{h_{k}(p)}{\mu}\right] .
\end{aligned}
$$

Linearizing these two equations around the steady-state, and denoting $\tilde{x}=\tilde{K}, \tilde{p}$ the long-term values of $x=K, p$, we obtain in a matrix form:

$$
(\dot{K}, \dot{p})^{T}=J(K(t)-\tilde{K}, p(t)-\tilde{p})^{T}
$$

where $J$ is given by

$$
J \equiv\left(\begin{array}{ll}
b_{11} & b_{12} \\
b_{21} & b_{22}
\end{array}\right)
$$

with

$$
\begin{aligned}
& b_{11}=\frac{Y_{K}^{N}}{\mu}-\delta_{K}=\frac{\tilde{h}}{\mu\left(\tilde{k}^{N}-\tilde{k}_{T}\right)}-\delta_{K} \gtrless 0, \quad b_{12}=\frac{Y_{p}^{N}}{\mu}-c_{p}^{N}>0, \\
& b_{21}=0, \quad b_{22}=-\tilde{p} \frac{h_{k k} k_{p}^{N}}{\mu}=-\frac{\tilde{f}}{\tilde{p}\left(\tilde{k}^{N}-\tilde{k}^{T}\right)}=\frac{Y_{K}^{T}}{\tilde{p}} \lessgtr 0 .
\end{aligned}
$$




\section{Equilibrium Dynamics}

By denoting $\nu$ the eigenvalue of matrix $J$, the characteristic equation for the matrix of the linearized system (61) can be written as follows:

$$
\nu^{2}-\frac{1}{\tilde{p} \mu}\left(\mu Y_{K}^{T}+\tilde{p} Y_{K}^{N}\right) \nu+\frac{Y_{K}^{T}}{\tilde{p}}\left(\frac{Y_{K}^{N}}{\mu}-\delta_{K}\right)=0
$$

where we used the fact that $-\frac{\tilde{p} h_{k k} k_{p}^{N}}{\mu}=\frac{Y_{K}^{T}}{\tilde{p}}$.

The determinant denoted by Det of the linearized $2 \times 2$ matrix (61) is unambiguously negative: ${ }^{1}$

$$
\text { Det } \mathrm{J}=b_{11} b_{22}=\frac{Y_{K}^{T}}{\tilde{p}}\left(\frac{Y_{K}^{N}}{\mu}-\delta_{K}\right)=-\frac{\tilde{f} \tilde{h}}{\mu \tilde{p}\left(\tilde{k}^{N}-\tilde{k}^{T}\right)^{2}}-\delta_{K} \frac{Y_{K}^{T}}{\tilde{p}}<0,
$$

and the trace denoted by $\operatorname{Tr}$ given by

$$
\operatorname{Tr} \mathrm{J}=b_{11}+b_{22}=\frac{1}{\tilde{p} \mu}\left(\mu Y_{K}^{T}+\tilde{p} Y_{K}^{N}\right)-\delta_{K}=\frac{h_{k}}{\mu}-\delta_{K}=r^{\star}>0,
$$

where we used the fact that at the long-run equilibrium $\frac{h_{k}}{\mu}=r^{\star}+\delta_{K}$.

From (61), the characteristic root obtained from $J$ writes as follows:

$$
\nu_{i} \equiv \frac{1}{2}\left\{r^{\star} \pm \sqrt{\left(r^{\star}\right)^{2}-4 \frac{Y_{K}^{T}}{\tilde{p}}\left(\frac{Y_{K}^{N}}{\mu}-\delta_{K}\right)}\right\} \gtrless 0, \quad i=1,2 .
$$

Using (66), then (67) can be rewritten as follows:

$$
\nu_{i} \equiv \frac{1}{2}\left\{r^{\star} \pm\left[\frac{Y_{K}^{T}}{\tilde{p}}-\left(\frac{Y_{K}^{N}}{\mu}-\delta_{K}\right)\right]\right\} \gtrless 0, \quad i=1,2 .
$$

We denote by $\nu_{1}<0$ and $\nu_{2}>0$ the stable and unstable real eigenvalues, satisfying

$$
\nu_{1}<0<r^{\star}<\nu_{2}
$$

Since the system features one state variable, $K$, and one jump variable, $p$, the equilibrium yields a unique one-dimensional stable saddle-path.

\section{Formal Solutions}

General solutions paths are given by :

$$
\begin{aligned}
K(t)-\tilde{K} & =B_{1} e^{\nu_{1} t}+B_{2} e^{\nu_{2} t}, \\
p(t)-\tilde{p} & =\omega_{2}^{1} B_{1} e^{\nu_{1} t}+\omega_{2}^{2} B_{2} e^{\nu_{2} t},
\end{aligned}
$$

where we normalized $\omega_{1}^{i}$ to unity. The eigenvector $\omega_{2}^{i}$ associated with eigenvalue $\mu_{i}$ is given by

$$
\omega_{2}^{i}=\frac{\nu_{i}-b_{11}}{b_{12}}
$$

\footnotetext{
${ }^{1}$ Starting from the equality of labor marginal products between sectors, using the fact that $f_{k}=p h_{k}$ and $h_{k}=r^{\star}+\delta_{K}$, it is straightforward to prove that $b_{11}$ is positive in the case $k^{N}>k^{T}$.
} 
with

$$
\begin{aligned}
b_{11} & =\frac{Y_{K}^{N}}{\mu}-\delta_{K}=\frac{\tilde{h}}{\mu\left(\tilde{k}^{N}-\tilde{k}_{T}\right)}-\delta_{K} \gtrless 0, \\
b_{12} & =\frac{Y_{p}^{N}}{\mu}-c_{p}^{N}>0,
\end{aligned}
$$

where $c_{p}^{N}$ is given by (47e).

Case $k^{N}>k^{T}$

This assumption reflects the fact that the capital-labor ratio of the non traded good sector exceeds the capital-labor of the traded sector. From (68), the stable and unstable eigenvalues can be rewritten as follows:

$$
\begin{aligned}
\nu_{1} & =-\frac{\tilde{f}}{\tilde{p}\left(\tilde{k}^{N}-\tilde{k}^{T}\right)}<0, \\
\nu_{2} & =\frac{\tilde{h}}{\mu\left(\tilde{k}^{N}-\tilde{k}^{T}\right)}-\delta_{K}>0,
\end{aligned}
$$

since we suppose that $k^{N}>k^{T}$.

We can deduce the signs of several useful expressions:

$$
\begin{aligned}
Y_{K}^{N} & =\mu\left(\nu_{2}+\delta_{K}\right)>0, \\
Y_{K}^{T} & =\tilde{p} \nu_{1}<0 \\
\frac{\tilde{p} h_{k k} k_{p}^{N}}{\mu} & =-\nu_{1}>0 \\
Y_{\tau^{F}}^{N} & =\tilde{k}^{T}\left(\nu_{2}+\delta_{K}\right) \sigma_{L} \tilde{L} \frac{\tilde{\Lambda}}{\left(1+\tau^{F}\right)}>0, \\
Y_{\tau^{F}}^{T} & =\tilde{p} \tilde{k}^{N} \nu_{1} \sigma_{L} \tilde{L} \frac{\tilde{\Lambda}}{\left(1+\tau^{F}\right)}<0, \\
Y_{\bar{\lambda}}^{N} & =-\frac{1}{\bar{\lambda}} \sigma_{L} \tilde{L} \tilde{k}^{T} \mu\left(\nu_{2}+\delta_{K}\right)<0, \\
Y_{\bar{\lambda}}^{T} & =-\frac{1}{\bar{\lambda}} \sigma_{L} \tilde{L} \tilde{p} \tilde{k}^{N} \nu_{1}>0 .
\end{aligned}
$$

We write out eigenvector $\omega^{i}$, corresponding with stable eigenvalue $\nu_{1}$ with $i=1,2$, to determine their signs:

$$
\omega^{1}=\left(\begin{array}{cc}
1 & (+) \\
\frac{\nu_{1}-\nu_{2}}{\left(\frac{Y_{p}^{N}}{\mu}-c_{p}^{N}\right)} & (-)
\end{array}\right), \quad \omega^{2}=\left(\begin{array}{ll}
1 & (+) \\
0 &
\end{array}\right) .
$$

Case $k^{T}>k^{N}$

This assumption reflects the fact that the capital-labor ratio of the traded good sector exceeds the capital-labor ratio of the non traded sector. From (68), the stable and unstable 
eigenvalues can be rewritten as follows:

$$
\begin{aligned}
\nu_{1} & =\frac{\tilde{h}}{\mu\left(\tilde{k}^{N}-\tilde{k}^{T}\right)}-\delta_{K}<0, \\
\nu_{2} & =-\frac{\tilde{f}}{\tilde{p}\left(\tilde{k}^{N}-\tilde{k}^{T}\right)}>0,
\end{aligned}
$$

since we suppose that $k^{T}>k^{N}$.

We can deduce the signs of several useful expressions:

$$
\begin{aligned}
Y_{K}^{N} & =\mu\left(\nu_{1}+\delta_{K}\right)<0 \\
Y_{K}^{T} & =\tilde{p} \nu_{2}>0, \\
\frac{\tilde{p} h_{k k} k_{p}^{N}}{\mu} & =-\nu_{2}<0 \\
Y_{\tau^{F}}^{N} & =\tilde{k}^{T} \mu\left(\nu_{1}+\delta_{K}\right) \sigma_{L} \tilde{L} \frac{\tilde{\Lambda}}{\left(1+\tau^{F}\right)}<0, \\
Y_{\tau^{F}}^{T} & =\tilde{p} \tilde{k}^{N} \nu_{2} \sigma_{L} \tilde{L} \frac{\tilde{\Lambda}}{\left(1+\tau^{F}\right)}>0, \\
Y_{\bar{\lambda}}^{N} & =-\frac{1}{\bar{\lambda}} \sigma_{L} \tilde{L} \tilde{k}^{T} \mu\left(\nu_{1}+\delta_{K}\right)>0, \\
Y_{\bar{\lambda}}^{T} & =-\frac{1}{\bar{\lambda}} \sigma_{L} \tilde{L} \tilde{p} \tilde{k}^{N} \nu_{2}<0 .
\end{aligned}
$$

We write out the four eigenvectors $\omega^{i}$, corresponding with stable eigenvalues $\nu_{i}$ with $i=$ 1,2 , to determine their signs:

$$
\omega^{1}=\left(\begin{array}{cc}
1 & (+) \\
0 &
\end{array}\right), \quad \omega^{2}=\left(\begin{array}{c}
0 \\
\frac{\nu_{2}-\nu_{1}}{\left(\frac{Y_{p}^{N}}{\mu}-c_{p}^{N}\right)}
\end{array} \quad(+)\right) .
$$

\section{Formal Solution for the Stock of Foreign Assets}

We first linearize equation (13j) around the steady-state:

$$
\dot{b}(t)=r^{\star}(b(t)-\tilde{b})+Y_{K}^{T}(K(t)-\tilde{K})+\left[Y_{p}^{T}-c_{p}^{T}\right](p(t)-\tilde{p}) .
$$

where $c_{p}^{T}$ is given by $(47 \mathrm{~b})$.

Inserting general solutions for $K(t)$ and $p(t)$, the solution for the stock of international assets writes as follows:

$$
\dot{b}(t)=r^{\star}(b(t)-\tilde{b})+Y_{K}^{T} \sum_{i=1}^{2} B_{i} e^{\nu_{i} t}+\left[Y_{p}^{T}-c_{p}^{T}\right] \sum_{i=1}^{2} B_{i} \omega_{2}^{i} e^{\nu_{i} t} .
$$

Solving the differential equation leads to the following expression:

$$
b(t)-\tilde{b}=\left[\left(b_{0}-\tilde{b}\right)-\Phi_{1} B_{1}-\Phi_{2} B_{2}\right] e^{r^{\star} t}+\Phi_{1} B_{1} e^{\nu_{1} t}+\Phi_{2} B_{2} e^{\nu_{2} t},
$$


with

$$
\Phi_{i}=\frac{N_{i}}{\nu_{i}-r^{\star}}=\frac{Y_{K}^{T}+\left[Y_{p}^{T}-c_{p}^{T}\right] \omega_{2}^{i}}{\nu_{i}-r^{\star}}, \quad i=1,2 .
$$

Invoking the transversality condition for intertemporal solvency, i. e. equation (14), the terms in brackets of equation (81) must be null and we must set $B_{2}=0$. We obtain the linearized version of the nation's intertemporal budget constraint:

$$
b_{0}-\tilde{b}=\Phi_{1} B_{1}
$$

The stable solution for net foreign assets finally reduces to:

$$
b(t)-\tilde{b}=\Phi_{1} B_{1}
$$

Inserting the value for the constant $B_{1}$ given by the stable solution for $K(t)$ by setting $t=0$, we can rewrite the national intertemporal budget constraint as a function of initial capital stock:

$$
b_{0}-\tilde{b}=\Phi_{1}\left(K_{0}-\tilde{K}\right) .
$$

Case $k^{N}>k^{T}$

$$
\begin{aligned}
N_{1} & =Y_{K}^{T}+\left(Y_{p}^{T}-c_{p}^{T}\right) \omega_{2}^{1}, \\
& =\tilde{p} \nu_{2}\left\{1+\frac{\omega_{2}^{1}}{\tilde{p} \nu_{2}}\left[\sigma_{c} \tilde{c}^{N}-\sigma_{L} \tilde{L} \tilde{k}^{T}\left(\nu_{2}+\delta_{K}\right) \tilde{\Lambda}\right]\right\} \gtrless 0, \\
N_{2} & =Y_{K}^{T}+\left(Y_{p}^{T}-c_{p}^{T}\right) \omega_{2}^{2}, \\
& =Y_{K}^{T}=\tilde{p} \nu_{1}<0,
\end{aligned}
$$

where (86c) follows from the fact that $\omega_{2}^{2}=0$. We made use of property (57a) together with the fact that $c_{p}^{T}=p_{c} c_{p}-p c_{p}^{N}$ to compute $Y_{p}^{T}-c_{p}^{T}=-\tilde{p}\left(\frac{Y_{p}^{N}}{\mu}-c_{p}^{N}\right)-p_{c} c_{p}-\sigma_{L} \tilde{L} \tilde{k}^{T}\left(\nu_{2}+\delta_{K}\right) \tilde{\Lambda} \gtrless 0$.

The sign of $\Phi_{1}$ is ambiguous and reflects the impact of the capital accumulation on the net foreign assets accumulation along a stable transitional path:

$$
\dot{b}(t)=\Phi_{1} \dot{K}(t) .
$$

where $\dot{K}(t)=\nu_{1} B_{1} e^{\nu_{1} t}$. Following empirical evidence suggesting that the current account and investment are negatively correlated (see e. g. Glick and Rogoff [?]), we will impose thereafter:

Assumption $1 \Phi_{1}<0$ which implies that $N_{1}>0$.

The condition for the assumption to hold, i. e. $N_{1}>0$, may be rewritten as follows:

$$
\nu_{2}>-\frac{\omega_{2}^{1}}{\tilde{p}}\left[\sigma_{c} \tilde{c}^{N}-\sigma_{L} \tilde{L} \tilde{k}^{T}\left(\nu_{2}+\delta_{K}\right) \tilde{\Lambda}\right]
$$


Case $k^{T}>k^{N}$

$$
\begin{aligned}
N_{1} & =Y_{K}^{T}+\left(Y_{p}^{T}-c_{p}^{T}\right) \omega_{2}^{1}, \\
& =Y_{K}^{T}=\tilde{p} \nu_{2}>0, \\
N_{2} & =Y_{K}^{T}+\left(Y_{p}^{T}-c_{p}^{T}\right) \omega_{2}^{2}, \\
& =\tilde{p} \nu_{1}\left\{1+\frac{\omega_{2}^{2}}{\tilde{p} \nu_{1}}\left[\sigma_{c} \tilde{c}^{N}-\sigma_{L} \tilde{L} \tilde{k}^{T}\left(\nu_{1}+\delta_{k}\right) \tilde{\Lambda}\right]\right\}, \lessgtr 0,
\end{aligned}
$$

where (88a) follows from the fact that $\omega_{2}^{1}=0$. We made use of property (57a) together with $c_{p}^{T}=p_{c} c_{p}-p c_{p}^{N}$ to compute $Y_{p}^{T}-c_{p}^{T}=-\tilde{p}\left(\frac{Y_{p}^{N}}{\mu}-c_{p}^{N}\right)-p_{c} c_{p}-\sigma_{L} \tilde{L} \tilde{k}^{T}\left(\nu_{1}+\delta_{K}\right) \gtrless 0$.

\section{Derivation of the Current Account Equation}

This section is dedicated to the determination of current account law of motion. Substituting the definition of transfer $Z$ to households from the government derived from (??) together with the market clearing condition for non traded goods (13i) into (6) we get:

$$
\begin{aligned}
\dot{b} & =r^{\star} b(t)+\left(r^{K}+\delta_{K}\right) K(t)+w^{A} L(t)-p_{c}\left(1+\tau^{c}\right) c(t)-p(t) I(t)+Z, \\
& =r^{\star} b+\left(r^{K} K+w^{F} L\right)-p_{c} c-p\left(\frac{Y^{N}}{\mu}-c^{N}-g^{N}\right) .
\end{aligned}
$$

Using the fact that $L^{T}+L^{N}=L, K^{T}+K^{N}=K$, the dynamic equation for the current account can be rewritten as follows:

$$
\begin{aligned}
\dot{b} & =r^{\star} b+\left[w^{F} L^{T}+\left(r^{K}+\delta_{K}\right) K^{T}\right]+\left[w^{F} L^{N}+\left(r^{K}+\delta_{K}\right) K^{N}\right]-p Y^{N}-c^{T}-g^{T}, \\
& =r^{\star} b+Y^{T}-c^{T}-g^{T},
\end{aligned}
$$

where the overall variable cost $w^{F} L^{N}+\left(r^{K}+\delta_{K}\right) K^{N}$ in the non traded sector and output net of fixed cost in that sector, i. e. $p \frac{Y^{N}}{\mu}=p Z^{N}$, cancel each other. ${ }^{2}$

\section{Long-Run Effects of Unanticipated Permanent Tax Shocks}

In this section, we calculate formal expressions of steady-state changes. For clarity purpose, we assume that $\delta_{K}=0$ since it does not modify qualitatively the long-run effects of tax policies. This assumption will be relaxed in numerical analysis. We totally differentiate the

\footnotetext{
${ }^{2}$ In the traded sector which is perfectly competitive, we have : $Y^{T}=F_{L} L^{T}+\left(r^{K}+\delta_{K}\right) K^{T}=w^{F} L^{T}+$ $\left(r^{K}+\delta_{K}\right) K^{T}$. Instead, in the non traded sector which is imperfectly competitive we have: $p Z^{N}=p \frac{H_{L}}{\mu} L^{N}+$ $p \frac{H_{K}}{\mu} K^{N}$ or $p \mu Z^{N}=p Y^{N}=p H_{L} L^{N}+p H_{K} K^{N}=w^{F} L^{N}+\left(r^{K}+\delta_{K}\right) K^{N}$.
} 
system (22) evaluated at the steady-state which yields in a matrix form:

$$
\left(\begin{array}{cccc}
\frac{h_{k k} k_{p}^{N}}{\mu} & 0 & 0 & 0 \\
\left(\frac{Y_{p}^{N}}{\mu}-c_{p}^{N}\right) & \frac{Y_{K}^{N}}{\mu} & \left(\frac{Y_{\bar{\lambda}}^{N}}{\mu}-c_{\bar{\lambda}}^{N}\right) & 0 \\
\left(Y_{p}^{T}-c_{p}^{T}\right) & Y_{K}^{T} & \left(Y_{\bar{\lambda}}^{T}-c_{\bar{\lambda}}^{T}\right) & r^{\star} \\
0 & -\Phi_{1} & 0 & 1
\end{array}\right)\left(\begin{array}{c}
\mathrm{d} \tilde{p} \\
\mathrm{~d} \tilde{K} \\
\mathrm{~d} \bar{\lambda} \\
\mathrm{d} \tilde{b}
\end{array}\right)=\left(\begin{array}{c}
0 \\
-\frac{Y_{\tau^{F}}^{N}}{\mu} \mathrm{d} \tau^{F}-\frac{Y_{\tau^{H}}^{N}}{\mu} \mathrm{d} \tau^{H}+c_{\tau^{c}}^{N} \mathrm{~d} \tau^{c} \\
-Y_{\tau^{F}}^{T} \mathrm{~d} \tau^{F}-Y_{\tau^{H}}^{T} \mathrm{~d} \tau^{H}+c_{\tau^{c}}^{T} \mathrm{~d} \tau^{c} \\
0
\end{array}\right)
$$

The determinant denoted by $D$ of the matrix of coefficients is given by:

$$
D \equiv \frac{h_{k k} k_{p}^{N}}{\mu}\left\{\frac{Y_{K}^{N}}{\mu}\left(Y_{\bar{\lambda}}^{T}-c_{\bar{\lambda}}^{T}\right)-\left(\frac{Y_{\bar{\lambda}}^{N}}{\mu}-c_{\bar{\lambda}}^{N}\right)\left[Y_{K}^{T}+r^{\star} \Phi_{1}\right]\right\}
$$

We have to consider two cases, depending on wether the non traded sector is more or less capital intensive than the traded sector:

$$
\begin{aligned}
D= & -\frac{\nu_{1} \nu_{2}}{\tilde{p} \bar{\lambda}}\left(\sigma_{L} \tilde{w}^{F} \tilde{L}+\sigma_{c} p_{c} \tilde{c}\right)>0, \text { case } k^{T}>k^{N}, \\
D= & -\frac{\nu_{1} \nu_{2}}{\tilde{p} \bar{\lambda}}\left\{\left(\sigma_{L} \tilde{w}^{F} \tilde{L}+\sigma_{c} p_{c} \tilde{c}\right)+\frac{r^{\star}}{\nu_{2}} \frac{\omega_{2}^{1}}{\nu_{2}}\left(\sigma_{c} \tilde{c}^{N}-\sigma_{L} \tilde{L} \tilde{k}^{T} \nu_{2}\right)\left(\sigma_{c} \tilde{c}^{N}-\sigma_{L} \tilde{L} \tilde{k}^{T} \nu_{2} \tilde{\Lambda}\right)\right\} \\
& \quad \text { case } k^{N}>k^{T},
\end{aligned}
$$

where we used the fact that and $\mu f k^{N}-p h k^{T}=\mu w^{F}\left(k^{N}-k^{T}\right)$ together with $-p\left(k^{N} \nu_{2}+k^{T} \nu_{1}\right) \equiv w^{F}$ if $k^{T}>k^{N}$ or $-p\left(k^{N} \nu_{1}+k^{T} \nu_{2}\right) \equiv w^{F}$ if $k^{N}>k^{T}$.

\section{Useful Expressions}

We have computed these useful expressions:

$$
\begin{aligned}
\frac{Y_{K}^{N}}{\mu} Y_{\bar{\lambda}}^{T}-Y_{K}^{T} \frac{Y_{\bar{\lambda}}^{N}}{\mu} & =\sigma_{L} \frac{\tilde{\bar{\lambda}}}{\left(\tilde{k}^{N}-\tilde{k}^{T}\right)}, \\
p_{c}^{\prime} Y_{K}^{T}-\left(1-\alpha_{c}\right) p_{c} \frac{Y_{K}^{N}}{\mu} & =-\frac{p_{c}}{\tilde{p}}\left[\frac{\alpha_{c} \tilde{f}+\left(1-\alpha_{c}\right) \tilde{p} \tilde{h}}{\left(\tilde{k}^{N}-\tilde{k}^{T}\right)}\right], \\
\frac{Y_{\bar{\lambda}}^{N}}{\mu}-c_{\bar{\lambda}}^{N} & =\frac{1}{\bar{\lambda}}\left[-\sigma_{L} \tilde{L} \tilde{k}^{T} \frac{\tilde{h}}{\left(\tilde{k}^{N}-\tilde{k}^{T}\right)}+\sigma_{c} \tilde{c}^{N}\right], \\
Y_{K}^{T}-c_{\bar{\lambda}}^{T} & =\frac{\tilde{p}}{\bar{\lambda}}\left[\sigma_{L} \tilde{L} \tilde{k}^{N} \frac{\tilde{f}}{\tilde{p}\left(\tilde{k}^{N}-\tilde{k}^{T}\right)}+\sigma_{c} \frac{\tilde{c}^{T}}{\tilde{p}}\right] .
\end{aligned}
$$


In the case $\underline{k^{N}>k^{T}}$, useful expressions (99) write as follows:

$$
\begin{aligned}
\frac{Y_{K}^{N}}{\mu} Y_{\bar{\lambda}}^{T}-Y_{K}^{T} \frac{Y_{\bar{\lambda}}^{N}}{\mu} & =-\tilde{p} \nu_{1} \nu_{2} \frac{\sigma_{L} \tilde{L}}{\bar{\lambda}}\left(\tilde{k}^{N}-\tilde{k}^{T}\right)>0, \\
p_{c}^{\prime} Y_{K}^{T}-\left(1-\alpha_{c}\right) p_{c} \frac{Y_{K}^{N}}{\mu} & =-p_{c}\left[\nu_{2}-\alpha_{c} r^{\star}\right]<0, \\
\frac{Y_{\bar{\lambda}}^{N}}{\mu}-c_{\bar{\lambda}}^{N} & =-\frac{1}{\bar{\lambda}}\left[\sigma_{L} \tilde{L} \tilde{k}^{T} \nu_{2}-\sigma_{c} \tilde{c}^{N}\right]<0, \\
Y_{\bar{\lambda}}^{T}-c_{\bar{\lambda}}^{T} & =-\frac{1}{\bar{\lambda}}\left[\sigma_{L} \tilde{L} \tilde{p} \tilde{k}^{N} \nu_{1}-\sigma_{c} \tilde{c}^{T}\right]>0, \\
\frac{Y_{K}^{N}}{\mu} c_{\tau^{c}}^{T}-Y_{K}^{T} c_{\tau^{c}}^{N} & =\frac{\sigma_{c} p_{c} \tilde{c}}{\left(1+\tau^{c}\right)}\left[-\nu_{2}\left(1-\alpha_{c}\right)+\nu_{1} \alpha_{c}\right]<0, \\
Y_{K}^{T} \frac{Y_{\tau^{F}}^{N}}{\mu}-\frac{Y_{K}^{N}}{\mu} Y_{\tau^{F}}^{T} & =-\tilde{p} \nu_{1} \nu_{2} \sigma_{L} \tilde{L} \frac{\tilde{\Lambda}}{\left(1+\tau^{F}\right)}\left(\tilde{k}^{N}-\tilde{k}^{T}\right)>0, \\
Y_{K}^{T} \frac{Y_{\tau^{H}}^{N}}{\mu}-\frac{Y_{K}^{N}}{\mu} Y_{\tau^{H}}^{T} & =-\tilde{p} \nu_{1} \nu_{2} \sigma_{L} \tilde{L} \frac{(\tilde{w}-\kappa)}{\tilde{w}^{A}}\left(\tilde{k}^{N}-\tilde{k}^{T}\right)>0, \\
Y_{p}^{T}-c_{p}^{T} & =\left(\sigma_{c} \tilde{c}^{N}-\sigma_{L} \tilde{L} \tilde{\Lambda} \tilde{k}^{T} \nu_{2}\right)-\tilde{p}\left(\frac{Y_{p}^{N}}{\mu}-c_{p}^{N}\right) .
\end{aligned}
$$

where we used the fact that $Y_{p}^{T}=-\tilde{p} \frac{Y_{p}^{N}}{\mu}-\sigma_{L} \tilde{L} \tilde{\Lambda} \tilde{k}^{T} \nu_{2}, c_{p}^{T}=p_{c} c_{p}-\tilde{p} c_{p}^{N}$ and $p_{c} c_{p}=-\sigma_{c} \tilde{c}^{N}$ to rewrite $Y_{p}^{T}-c_{p}^{T}($ see $(93 \mathrm{~h}))$.

In the case $\underline{k^{T}>k^{N}}$, useful expressions (99) write as follows:

$$
\begin{aligned}
\frac{Y_{K}^{N}}{\mu} Y_{\bar{\lambda}}^{T}-Y_{K}^{T} \frac{Y_{\bar{\lambda}}^{N}}{\mu} & =\tilde{p} \nu_{1} \nu_{2} \frac{\sigma_{L} \tilde{L}}{\bar{\lambda}}\left(\tilde{k}^{T}-\tilde{k}^{N}\right)<0, \\
p_{c}^{\prime} Y_{K}^{T}-\left(1-\alpha_{c}\right) p_{c} Y_{K}^{N} & =-p_{c}\left[\nu_{1}-\alpha_{c} r^{\star}\right]>0, \\
\frac{Y_{\bar{\lambda}}^{N}}{\mu}-c_{\bar{\lambda}}^{N} & =-\frac{1}{\bar{\lambda}}\left(\sigma_{L} \tilde{L} \tilde{k}^{T} \nu_{1}-\sigma_{c} \tilde{c}^{N}\right)>0, \\
Y_{\bar{\lambda}}^{T}-c_{\bar{\lambda}}^{T} & =-\frac{1}{\bar{\lambda}}\left[\sigma_{L} \tilde{L} \tilde{p} \tilde{k}^{N} \nu_{2}-\sigma_{c} \tilde{c}^{T}\right] \gtrless 0, \\
\frac{Y_{K}^{N}}{\mu} c_{\tau^{c}}^{T}-Y_{K}^{T} c_{\tau^{c}}^{N} & =\frac{\sigma_{c} p_{c} \tilde{c}}{\left(1+\tau^{c}\right)}\left[-\nu_{1}\left(1-\alpha_{c}\right)+\nu_{2} \alpha_{c}\right]>0, \\
Y_{K}^{T} \frac{Y_{\tau^{F}}^{N}}{\mu}-\frac{Y_{K}^{N}}{\mu} Y_{\tau^{F}}^{T} & =\tilde{p} \nu_{1} \nu_{2} \sigma_{L} \tilde{L} \frac{\tilde{\Lambda}}{\left(1+\tau^{F}\right)}\left(\tilde{k}^{T}-\tilde{k}^{N}\right)<0, \\
Y_{p}^{T}-c_{p}^{T} & =\left(\sigma_{c} \tilde{c}^{N}-\sigma_{L} \tilde{L} \tilde{\Lambda} \tilde{k}^{T} \nu_{1}\right)-\tilde{p}\left(\frac{Y_{p}^{N}}{\mu}-c_{p}^{N}\right), \\
Y_{K}^{T}+r^{\star} \Phi_{1} & =-\tilde{p} \nu_{1} .
\end{aligned}
$$

\section{Long-Run Effects of an Unanticipated Permanent Change in the Consumption}

\section{Tax Rate}

case $k^{N}>k^{T}$ 


$$
\begin{aligned}
\frac{\mathrm{d} \tilde{c}}{\mathrm{~d} \tau^{c}} & =-\left(\frac{\sigma_{c} \tilde{c} \sigma_{L} \tilde{L}}{\Delta\left(1+\tau^{c}\right)}\right)\left[w^{F}-\tilde{k}^{T} \frac{r^{\star}}{\nu_{2}} \omega_{2}^{1}\left(\sigma_{c} \tilde{c}^{N}-\sigma_{L} \tilde{L} k^{T} \nu_{2} \tilde{\Lambda}\right)\right] \lessgtr 0, \\
\frac{\mathrm{d} \tilde{L}}{\mathrm{~d} \tau^{c}} & =-\left(\frac{\sigma_{c} p_{c} \tilde{c} \sigma_{L} \tilde{L}}{1+\tau^{c}}\right) \frac{1}{\Delta}\left[1+\alpha_{c} \frac{r^{\star}}{\nu_{2}} \frac{\omega_{2}^{1}}{\tilde{p} \nu_{2}}\left(\sigma_{c} \tilde{c}^{N}-\sigma_{L} \tilde{L} k^{T} \nu_{2} \tilde{\Lambda}\right)\right]<0, \\
\frac{\mathrm{d} \bar{\lambda}}{\mathrm{d} \tau^{c}} & =-\left(\frac{\sigma_{c} \bar{\lambda} p_{c} \tilde{c}}{1+\tau^{c}}\right) \frac{1}{\Delta}\left[1+\alpha_{c} \frac{r^{\star}}{\nu_{2}} \frac{\omega_{2}^{1}}{\tilde{p} \nu_{2}}\left(\sigma_{c} \tilde{c}^{N}-\sigma_{L} \tilde{L} k^{T} \nu_{2} \tilde{\Lambda}\right)\right]<0, \\
\frac{\mathrm{d} \tilde{p}}{\mathrm{~d} \tau^{c}} & =0, \\
\frac{\mathrm{d} \tilde{K}}{\mathrm{~d} \tau^{c}} & =\frac{1}{\nu_{2}}\left(\frac{1}{1+\tau^{c}}\right)\left(\frac{\sigma_{c} p_{c} \tilde{c} \sigma_{L} \tilde{L}}{\Delta}\right)\left[\alpha_{c} \tilde{k}^{N} \nu_{1}-\left(1-\alpha_{c}\right) \tilde{k}^{T} \nu_{2}\right]<0, \\
\frac{\mathrm{d} \tilde{b}}{\mathrm{~d} \tau^{c}} & =\Phi_{1} \frac{\mathrm{d} \tilde{K}}{\mathrm{~d} \tau^{c}>0,}
\end{aligned}
$$

where $\Delta=\left[\left(\sigma_{L} \tilde{w}^{F} \tilde{L}+\sigma_{c} p_{c} \tilde{c}\right)+\frac{r^{\star}}{\nu_{2}} \frac{\omega_{2}^{1}}{\nu_{2}}\left(\sigma_{c} \tilde{c}^{N}-\sigma_{L} \tilde{L} \tilde{k}^{T} \nu_{2}\right)\left(\sigma_{c} \tilde{c}^{N}-\sigma_{L} \tilde{L} \tilde{k}^{T} \nu_{2} \tilde{\Lambda}\right)\right]$ is assumed to be positive.

$$
\text { case } \begin{aligned}
k^{T}> & k^{N} \\
\frac{\mathrm{d} \tilde{c}}{\mathrm{~d} \tau^{c}} & =-\left(\frac{1}{1+\tau^{c}}\right)\left(\frac{\sigma_{c} \tilde{c} \sigma_{L} \tilde{w}^{F} \tilde{L}}{\sigma_{L} \tilde{w}^{F} \tilde{L}+\sigma_{c} p_{c} \tilde{c}}\right)<0, \\
\frac{\mathrm{d} \tilde{L}}{\mathrm{~d} \tau^{c}} & =-\left(\frac{1}{1+\tau^{c}}\right)\left(\frac{\sigma_{c} p_{c} \tilde{c} \sigma_{L} \tilde{L}}{\sigma_{L} \tilde{w}^{F} \tilde{L}+\sigma_{c} p_{c} \tilde{c}}\right)<0, \\
\frac{\mathrm{d} \bar{\lambda}}{\mathrm{d} \tau^{c}} & =-\bar{\lambda}\left(\frac{1}{1+\tau^{c}}\right)\left(\frac{\sigma_{c} p_{c} \tilde{c}}{\sigma_{L} \tilde{w}^{F} \tilde{L}+\sigma_{c} p_{c} \tilde{c}}\right)<0, \\
\frac{\mathrm{d} \tilde{p}}{\mathrm{~d} \tau^{c}} & =0, \\
\frac{\mathrm{d} \tilde{K}}{\mathrm{~d} \tau^{c}} & =\frac{1}{\nu_{1}}\left(\frac{1}{1+\tau^{c}}\right)\left(\frac{\sigma_{c} p_{c} \tilde{c} \sigma_{L} \tilde{L}}{\sigma_{L} \tilde{w}^{F} \tilde{L}+\sigma_{c} p_{c} \tilde{c}}\right)\left(\alpha_{c} \tilde{k}^{N} \nu_{2}-\left(1-\alpha_{c}\right) \tilde{k}^{T} \nu_{1}\right)<0, \\
\frac{\mathrm{d} \tilde{b}}{\mathrm{~d} \tau^{c}} & =-\tilde{p} \frac{\mathrm{d} \tilde{K}}{\mathrm{~d} \tau^{c}}>0 .
\end{aligned}
$$

Long-Run Effects of an Unanticipated Permanent Change in the Payroll Tax Rate

case $k^{N}>k^{T}$ 


$$
\begin{aligned}
\frac{\mathrm{d} \tilde{c}}{\mathrm{~d} \tau^{F}} & =-\sigma_{c} \tilde{c} \frac{\sigma_{L}}{\Delta} \frac{\tilde{L}}{\left(1+\tau^{F}\right)}\left[\tilde{w}^{F}-\tilde{k}^{T} r^{\star} \frac{\omega_{2}^{1}}{\nu_{2}}\left(\sigma_{c} \tilde{c}^{N}-\sigma_{L} \tilde{L} k^{T} \nu_{2} \tilde{\Lambda}\right)\right] \lessgtr 0, \\
\frac{\mathrm{d} \tilde{L}}{\mathrm{~d} \tau^{F}} & =-\frac{\sigma_{L} \tilde{L}}{\Delta} \frac{\tilde{\Lambda}}{\left(1+\tau^{F}\right)}\left\{\sigma_{c} p_{c} \tilde{c}+\frac{r^{\star}}{\nu_{2}} \frac{\omega_{2}^{1}}{\nu_{2}}\left(\sigma_{c} \tilde{c}^{N}-\sigma_{L} \tilde{L} k^{T} \nu_{2} \tilde{\Lambda}\right) \sigma_{c} \tilde{c}^{N}\right\}<0, \\
\frac{\mathrm{d} \bar{\lambda}}{\mathrm{d} \tau^{F}} & =\bar{\lambda} \frac{\sigma_{L} \tilde{L}}{\Delta} \frac{\tilde{\Lambda}}{\left(1+\tau^{F}\right)}\left[\tilde{w}^{F}-\tilde{k}^{T} r^{\star} \frac{\omega_{2}^{1}}{\nu_{2}}\left(\sigma_{c} \tilde{c}^{N}-\sigma_{L} \tilde{L} k^{T} \nu_{2} \tilde{\Lambda}\right)\right] \lessgtr 0, \\
\frac{\mathrm{d} \tilde{p}}{\mathrm{~d} \tau^{F}} & =0, \\
\frac{\mathrm{d} \tilde{K}}{\mathrm{~d} \tau^{F}} & =\frac{\sigma_{L} \tilde{L}}{\Delta \nu_{2}} \frac{\tilde{\Lambda}}{\left(1+\tau^{F}\right)} \sigma_{c} p_{c} \tilde{c}\left[\alpha_{c} \tilde{k}^{N} \nu_{1}-\left(1-\alpha_{c}\right) \tilde{k}^{T} \nu_{2}\right]<0, \\
\frac{\mathrm{d} \tilde{b}}{\mathrm{~d} \tau^{F}} & =\Phi_{1} \frac{\mathrm{d} \tilde{K}}{\mathrm{~d} \tau^{F}}>0,
\end{aligned}
$$

where we used the fact that $\tilde{p}\left(\nu_{2} \tilde{k}^{T}+\nu_{1} \tilde{k}^{N}\right)=-\tilde{p}\left(\tilde{h}-h_{k} \tilde{k}^{N}\right) \equiv-\tilde{w}^{F}$ and $L_{\tau^{H}}=$ $L_{\tau^{F}} \frac{(\tilde{w}-\kappa)}{\tilde{w}^{A}} \frac{1+\tau^{F}}{\tilde{\Lambda}}$.

case $k^{T}>k^{N}$

$$
\begin{aligned}
\frac{\mathrm{d} \tilde{c}}{\mathrm{~d} \tau^{F}} & =-\sigma_{c} \tilde{c} \frac{\sigma_{L} \tilde{L}}{\Delta} \frac{\tilde{\Lambda}}{\left(1+\tau^{F}\right)} \tilde{w}^{F}=\frac{1+\tau^{c}}{1+\tau^{F}} \frac{\mathrm{d} \tilde{c}}{\mathrm{~d} \tau^{c}}<0, \\
\frac{\mathrm{d} \tilde{L}}{\mathrm{~d} \tau^{F}} & =-\frac{\sigma_{L} \tilde{L}}{\Delta} \frac{\tilde{\Lambda}}{\left(1+\tau^{F}\right)} \sigma_{c} p_{c} \tilde{c}=\frac{1+\tau^{c}}{1+\tau^{F}} \frac{\mathrm{d} \tilde{L}}{\mathrm{~d} \tau^{c}}<0, \\
\frac{\mathrm{d} \bar{\lambda}}{\mathrm{d} \tau^{F}} & =\bar{\lambda} \frac{\sigma_{L} \tilde{L}}{\Delta} \frac{\tilde{\Lambda}}{\left(1+\tau^{F}\right)} \tilde{w}^{F}>0, \\
\frac{\mathrm{d} \tilde{p}}{\mathrm{~d} \tau^{F}} & =0, \\
\frac{\mathrm{d} \tilde{K}}{\mathrm{~d} \tau^{F}} & =\frac{\sigma_{L} \tilde{L}}{\nu_{1} \Delta} \frac{\tilde{\Lambda}}{\left(1+\tau^{F}\right)} \sigma_{c} p_{c} \tilde{c}\left[\alpha_{c} \tilde{k}^{N} \nu_{2}-\left(1-\alpha_{c}\right) \tilde{k}^{T} \nu_{1}\right]<0, \\
\frac{\mathrm{d} \tilde{b}}{\mathrm{~d} \tau^{F}} & =-\tilde{p} \frac{\mathrm{d} \tilde{K}}{\mathrm{~d} \tau^{F}}>0,
\end{aligned}
$$

where we let $\Delta \equiv \sigma_{L} \tilde{w}^{F} \tilde{L}+\sigma_{c} p_{c} \tilde{c}$ and we used the fact that $-\tilde{p}\left(\nu_{1} \tilde{k}^{T}+\nu_{2} \tilde{k}^{N}\right)=$ $\tilde{p}\left(\tilde{h}-h_{k} \tilde{k}^{N}\right) \equiv \tilde{w}^{F}$.

Long-Run Effects of an Unanticipated Permanent Change in the Wage Income Tax Rate

case $k^{N}>k^{T}$ 


$$
\begin{aligned}
\frac{\mathrm{d} \tilde{c}}{\mathrm{~d} \tau^{H}} & \left.=-\sigma_{c} \tilde{c} \frac{\sigma_{L}}{\Delta} \frac{\tilde{L}}{\tilde{w}^{A}}[\tilde{w}) \kappa \tilde{w}^{F}-\tilde{k}^{T} r^{\star} \frac{\omega_{2}^{1}}{\nu_{2}}\left(\sigma_{c} \tilde{c}^{N}-\sigma_{L} \tilde{L} k^{T} \nu_{2} \tilde{\Lambda}\right)\right] \lessgtr 0, \\
\frac{\mathrm{d} \tilde{L}}{\mathrm{~d} \tau^{H}} & =-\frac{\sigma_{L}}{\Delta} \frac{\tilde{L}}{(\tilde{w}-\kappa)} \tilde{w}^{A}\left\{\sigma_{c} p_{c} \tilde{c}+\frac{r^{\star}}{\nu_{2}} \frac{\omega_{2}^{1}}{\nu_{2}}\left(\sigma_{c} \tilde{c}^{N}-\sigma_{L} \tilde{L} k^{T} \nu_{2} \tilde{\Lambda}\right) \sigma_{c} \tilde{c}^{N}\right\}<0, \\
\frac{\mathrm{d} \bar{\lambda}}{\mathrm{d} \tau^{H}} & =\bar{\lambda} \frac{\sigma_{L} \tilde{L}}{\Delta} \frac{(\tilde{w}-\kappa)}{\tilde{w}^{A}}\left[\tilde{w}^{F}-\tilde{k}^{T} r^{\star} \frac{\omega_{2}^{1}}{\nu_{2}}\left(\sigma_{c} \tilde{c}^{N}-\sigma_{L} \tilde{L} k^{T} \nu_{2} \tilde{\Lambda}\right)\right] \lessgtr 0, \\
\frac{\mathrm{d} \tilde{p}}{\mathrm{~d} \tau^{H}} & =0, \\
\frac{\mathrm{d} \tilde{K}}{\mathrm{~d} \tau^{H}} & \left.=\frac{\sigma_{L} \tilde{L}}{\nu_{2} \Delta} \sigma_{c} p_{c} \tilde{c} \frac{(\tilde{w}-\kappa)}{\tilde{w}^{A}}\right)\left[\alpha_{c} \tilde{k}^{N} \nu_{1}-\left(1-\alpha_{c}\right) \tilde{k}^{T} \nu_{2}\right]<0, \\
\frac{\mathrm{d} \tilde{b}}{\mathrm{~d} \tau^{H}} & =-\Phi_{1} \frac{\mathrm{d} \tilde{K}}{\mathrm{~d} \tau^{H}}>0
\end{aligned}
$$

where we used the fact that $\frac{\tilde{w}\left(1-\tau^{H}\right)}{\tilde{w}^{A}}=\tilde{\Lambda}$.

case $k^{T}>k^{N}$

$$
\begin{aligned}
\frac{\mathrm{d} \tilde{c}}{\mathrm{~d} \tau^{H}} & =-\sigma_{c} \tilde{c} \frac{\sigma_{L}}{\Delta} \frac{\tilde{L}}{(\tilde{w}-\kappa)} \tilde{w}^{A}=\frac{\mathrm{d} \tilde{c}}{\mathrm{~d} \tau^{F}} \frac{(\tilde{w}-\kappa)}{\tilde{w}^{A}} \frac{1+\tau^{F}}{\tilde{\Lambda}}<0, \\
\frac{\mathrm{d} \tilde{L}}{\mathrm{~d} \tau^{H}} & =-\frac{\sigma_{L} \tilde{L}}{\Delta} \frac{(\tilde{w}-\kappa)}{\tilde{w}^{A}} \sigma_{c} p_{c} \tilde{c}<0, \\
\frac{\mathrm{d} \bar{\lambda}}{\mathrm{d} \tau^{H}} & =\bar{\lambda} \frac{\sigma_{L} \tilde{L}}{\Delta} \frac{(\tilde{w}-\kappa)}{\tilde{w}^{A}} \tilde{w}^{F}>0, \\
\frac{\mathrm{d} \tilde{p}}{\mathrm{~d} \tau^{H}} & =0, \\
\frac{\mathrm{d} \tilde{K}}{\mathrm{~d} \tau^{H}} & =\frac{\sigma_{L} \tilde{L}}{\nu_{1} \Delta} \frac{(\tilde{w}-\kappa)}{\tilde{w}^{A}} \sigma_{c} p_{c} \tilde{c}\left[\alpha_{c} \tilde{k}^{N} \nu_{2}-\left(1-\alpha_{c}\right) \tilde{k}^{T} \nu_{1}\right]<0, \\
\frac{\mathrm{d} \tilde{b}}{\mathrm{~d} \tau^{H}} & =-\tilde{p} \frac{\mathrm{d} \tilde{K}}{\mathrm{~d} \tau^{H}}>0,
\end{aligned}
$$

where we let $\Delta=\sigma_{L} \tilde{w}^{F} \tilde{L}+\sigma_{c} p_{c} \tilde{c}$.

\section{Inelastic Labor Supply Case: $\sigma_{L}=0$}

If labor is supplied inelastically, then the intertemporal elasticity of substitution for labor is null and total employment remains fixed to the level $\bar{L}=1$.

\section{Long-Run Effects of an Unanticipated Permanent Change of the Consumption}

\section{Tax Rate}

Set $\sigma_{L}=0$ into (95) or (96), we get:

$$
\begin{aligned}
\frac{\mathrm{d} \tilde{c}}{\mathrm{~d} \tau^{c}} & =\frac{\mathrm{d} \tilde{L}}{\mathrm{~d} \tau^{c}}=\frac{\mathrm{d} \tilde{p}}{\mathrm{~d} \tau^{c}}=\frac{\mathrm{d} \tilde{K}}{\mathrm{~d} \tau^{c}}=\frac{\mathrm{d} \tilde{b}}{\mathrm{~d} \tau^{c}}=0, \\
\frac{\mathrm{d} \bar{\lambda}}{\mathrm{d} \tau^{c}} & =-\frac{\bar{\lambda}}{\left(1+\tau^{c}\right)} .
\end{aligned}
$$

From (101a)-(101b), the elasticity of the marginal utility of wealth is equal to unity in absolute terms and the long-run levels of variables remain unaffected. A rise in consumption tax raises 
the marginal cost of current consumption. Since the trade-off between labor and leisure turns out to be irrelevant, total employment remains fixed such that $\bar{\lambda}$ must fall by the same proportion than the rise in $\tau^{c}$ thus leaving unaffected real consumption as the direct effect and the wealth effect cancel each other. Since demand for non tradables and tradables remain unaffected, capital stock and net foreign assets must not change for investment and the current account to be zero in the long-run. As the capital stock remains unchanged in the long-run, dynamics degenerate.

\section{Long-Run Effects of an Unanticipated Permanent Change of the Payroll Tax} Rate

Set $\sigma_{L}=0$ into (97) or (98), we get:

$$
\begin{aligned}
\frac{\mathrm{d} \tilde{c}}{\mathrm{~d} \tau^{F}} & =\frac{\mathrm{d} \tilde{L}}{\mathrm{~d} \tau^{F}}=\frac{\mathrm{d} \bar{\lambda}}{\mathrm{d} \tau^{F}}=\frac{\mathrm{d} \tilde{p}}{\mathrm{~d} \tau^{F}}=\frac{\mathrm{d} \tilde{K}}{\mathrm{~d} \tau^{F}}=\frac{\mathrm{d} \tilde{b}}{\mathrm{~d} \tau^{F}}=0, \\
\frac{\mathrm{d} \tilde{w}}{\mathrm{~d} \tau^{F}} & =-\frac{\tilde{w}}{\left(1+\tau^{F}\right)}<0 .
\end{aligned}
$$

From (102a)-(102b), a fall in $\tau^{F}$ leaves unchanged the steady-state levels of variables, and more importantly does no longer induce a wealth effect. The explanation is that whenever the trade-off between labor and leisure turns out to be irrelevant, total employment remains fixed. To insure that equality of sectoral labor marginal products holds, the wage must rise by the same proportion than the fall in the payroll tax. As the capital stock remains unchanged in the long-run, dynamics degenerate. In words, if labor is fixed, a change in the tax on wage paid by producers induces solely a direct effect on the wage rate.

\section{E The Two-Step Procedure: the Wealth Effect and the Direct Effects}

By analytical convenience, we rewrite the system of steady-state equations (22), assuming that $\delta_{K}=0$ :

$$
\begin{gathered}
\frac{h_{k}\left[k^{N}(\tilde{p})\right]}{\mu}=r^{\star}, \\
\frac{1}{\mu} Y^{N}\left(\tilde{K}, \tilde{p}, \bar{\lambda}, \tau^{F}, \tau^{H}\right)-c^{N}\left(\bar{\lambda}, \tilde{p}, \tau^{c}\right)-g^{N}=0, \\
r^{\star} \tilde{b}+Y^{T}\left(\tilde{K}, \tilde{p}, \bar{\lambda}, \tau^{F}, \tau^{H}\right)-c^{T}\left(\bar{\lambda}, \tilde{p}, \tau^{c}\right)-g^{T}=0,
\end{gathered}
$$

together with the intertemporal solvency condition

$$
\left(\tilde{b}-b_{0}\right)=\Phi_{1}\left(\tilde{K}-K_{0}\right) .
$$

where $K_{0}$ and $b_{0}$ correspond to the initially predetermined stocks of physical capital and foreign assets, the open economy starting from an initial steady-state at time $\mathcal{T}$. If the fiscal shock is permanent, then $\mathcal{T}=0$.

\section{Derivation of Steady-State Functions}


The new consistent procedure consists in two steps. In a first step, we solve the system (103a)-(103c) for $\tilde{p}, \tilde{K}$ and $\tilde{b}$ as functions of the marginal utility of wealth, $\bar{\lambda}$, the tax rates on consumption and labor together with the mark-up. Totally differentiating equations (103a)(103c) yields in matrix form:

$$
\begin{aligned}
& \left(\begin{array}{ccc}
h_{k k} k_{p}^{N} & 0 & 0 \\
\left(\frac{Y_{p}^{N}}{\mu}-c_{p}^{N}\right) & \frac{Y_{K}^{N}}{\mu} & 0 \\
\left(Y_{p}^{T}-c_{p}^{T}\right) & Y_{K}^{T} & r^{\star}
\end{array}\right)\left(\begin{array}{c}
\mathrm{d} \tilde{p} \\
\mathrm{~d} \tilde{K} \\
\mathrm{~d} \tilde{b}
\end{array}\right)= \\
& =\left(\begin{array}{c}
0 \\
-\left(\frac{Y_{\bar{\lambda}}^{N}}{\mu}-c_{\bar{\lambda}}^{N}\right) \mathrm{d} \bar{\lambda}+c_{\tau^{c}}^{N} \mathrm{~d} \tau^{c}-\frac{Y_{\tau^{F}}^{N}}{\mu} \mathrm{d} \tau^{F}-\frac{Y_{\tau^{H}}^{N}}{\mu} \mathrm{d} \tau^{H} \\
-\left(Y_{\bar{\lambda}}^{T}-c_{\bar{\lambda}}^{T}\right) \mathrm{d} \bar{\lambda}+c_{\tau^{c}}^{T} \mathrm{~d} \tau^{c}-Y_{\tau^{F}}^{T} \mathrm{~d} \tau^{F}-Y_{\tau^{H}}^{T} \mathrm{~d} \tau^{H}
\end{array}\right) .
\end{aligned}
$$

The equilibrium value of the marginal utility of wealth $\bar{\lambda}$ and tax rates, $\tau^{c}, \tau^{F}, \tau^{H}$ determine the following steady-state values:

$$
\begin{aligned}
\tilde{K} & =K\left(\bar{\lambda}, \tau^{c}, \tau^{F}, \tau^{H}\right), \\
\tilde{b} & =v\left(\bar{\lambda}, \tau^{c}, \tau^{F}, \tau^{H}\right),
\end{aligned}
$$

with partial derivatives given by:

$$
\begin{aligned}
K_{\bar{\lambda}} \equiv \frac{\partial \tilde{K}}{\partial \bar{\lambda}} & =-\frac{1}{\bar{\lambda}} \frac{1}{\nu_{1}}\left(\sigma_{c} \tilde{c}^{N}-\sigma_{L} \tilde{L} \tilde{k}^{T} \nu_{1}\right)>0 \quad \text { case } k^{T}>k^{N}, \\
& =-\frac{1}{\bar{\lambda}} \frac{1}{\nu_{2}}\left(\sigma_{c} \tilde{c}^{N}-\sigma_{L} \tilde{L} \tilde{k}^{T} \nu_{2}\right)>0 \quad \text { case } k^{N}>k^{T}, \\
v_{\bar{\lambda}} \equiv \frac{\partial \tilde{b}}{\partial \bar{\lambda}} & =-\frac{1}{\bar{\lambda}} \frac{1}{r^{\star} \tilde{h}}\left[\sigma_{c}\left(\tilde{f} \tilde{c}^{N}+\tilde{h} \tilde{c}^{T}\right)+\sigma_{L} \tilde{L} \tilde{h} \tilde{f}\right]<0,
\end{aligned}
$$

and

$$
\begin{aligned}
K_{\tau^{c}} \equiv \frac{\partial \tilde{K}}{\partial \tau^{c}} & =-\frac{1}{\nu_{1}}\left(\frac{\sigma_{c} \tilde{c}^{N}}{1+\tau^{c}}\right)>0 \quad \text { case } \quad k^{T}>k^{N} \\
& =-\frac{1}{\nu_{2}}\left(\frac{\sigma_{c} \tilde{c}^{N}}{1+\tau^{c}}\right)<0 \quad \text { case } \quad k^{N}>k^{T}, \\
v_{\tau^{c}} \equiv \frac{\partial \tilde{b}}{\partial \tau^{c}} & =-\frac{1}{r^{\star} \tilde{h}}\left(\frac{\sigma_{c}}{1+\tau^{c}}\right)\left(\tilde{f} \tilde{c}^{N}+\tilde{h} \tilde{c}^{T}\right)<0,
\end{aligned}
$$

and

$$
\begin{aligned}
K_{\tau^{F}} \equiv \frac{\partial \tilde{K}}{\partial \tau^{F}} & =-\frac{\sigma_{L} \tilde{L}}{1+\tau^{F}} \tilde{k}^{T}<0, \\
v_{\tau^{F}} \equiv \frac{\partial \tilde{b}}{\partial \tau^{F}} & =\frac{\tilde{f}}{r^{\star}} \frac{\sigma_{L} \tilde{L}}{1+\tau^{F}}>0 .
\end{aligned}
$$

Since real consumption and labor supply turn to be affected by the change in the capital income tax rate through the steady-state change of the real exchange rate, it is convenient to write their steady-state functions

$$
c=m\left(\bar{\lambda}, \tau^{c},\right), \quad L=n\left(\bar{\lambda}, \tau^{F}, \tau^{H},\right)
$$


where partial derivatives are given by (45) evaluated at the steady-state (that's why we substitute respectively the notations $m$ and $n$ for $c$ and $L$ ).

\section{Derivation of the Equilibrium Value of the Marginal Utility of Wealth}

The second step consists to determine the equilibrium change of $\bar{\lambda}$ by taking the total differential of the intertemporal solvency condition (103d):

$$
\left[v_{\bar{\lambda}}-\Phi_{1} K_{\lambda}\right] \mathrm{d} \bar{\lambda}=-\left[v_{\tau^{c}}-\Phi_{1} K_{\tau^{c}}\right] \mathrm{d} \tau^{c}-\left[v_{\tau^{F}}-\Phi_{1} K_{\tau^{F}}\right] \mathrm{d} \tau^{F}-\left[v_{\tau^{H}}-\Phi_{1} K_{\tau^{H}}\right] \mathrm{d} \tau^{H}
$$

from which may solve for the equilibrium value of $\bar{\lambda}$ as a function of tax rates:

$$
\bar{\lambda}=\lambda\left(\tau^{c}, \tau^{F}, \tau^{H}\right),
$$

with

$$
\begin{aligned}
\lambda_{\tau^{c}} \equiv \frac{\partial \bar{\lambda}}{\partial \tau^{c}} & =-\frac{\left[v_{\tau^{c}}-\Phi_{1} K_{\tau^{c}}\right]}{\left[v_{\bar{\lambda}}-\Phi_{1} K_{\bar{\lambda}}\right]}, \\
\lambda_{\tau^{F}} \equiv \frac{\partial \bar{\lambda}}{\partial \tau^{F}} & =-\frac{\left[v_{\tau^{F}}-\Phi_{1} K_{\tau^{F}}\right]}{\left[v_{\bar{\lambda}}-\Phi_{1} K_{\bar{\lambda}}\right]}, \\
\lambda_{\tau^{H}} \equiv \frac{\partial \bar{\lambda}}{\partial \tau^{H}} & =-\frac{\left[v_{\tau^{H}}-\Phi_{1} K_{\tau^{H}}\right]}{\left[v_{\bar{\lambda}}-\Phi_{1} K_{\bar{\lambda}}\right]} .
\end{aligned}
$$

\section{F Overall Effect of a Tax Reform}

We first substitute short-run static solutions for consumption, wage and labor given by (??) and (16), into the balanced government budget constraint (12) evaluated at the steady-state:

$$
\tau^{c} p_{c}(\tilde{p}) c\left(\bar{\lambda}, \tilde{p}, \tau^{c}\right)+\left[\left(\tau^{F}+\tau^{H}\right) w\left(\tilde{p}, \tau^{F}\right)-\tau^{H} \kappa\right] L\left(\bar{\lambda}, \tilde{p}, \tau^{F}, \tau^{H}\right)=Z,
$$

keeping in mind that the long-run value of the real exchange rate is unaffected by fiscal tax changes and $\bar{\lambda}=\lambda\left(\tau^{c}, \tau^{F}, \tau^{H}\right)$.

\section{F.1 Steady-State Changes of a Tax Reform: Substitution of Payroll Taxes for Consumption Taxes}

In this section, we estimate the long-run effects of a fall in the payroll $\operatorname{tax} \tau^{F}$ associated with a rise in the consumption tax rate $\tau^{c}$, which is adjusted accordingly to balance the government budget. Additionally, we assume that taxes on labor income are progressive so that $\kappa>0$ and $\Lambda<1$. To avoid confusion, we denote by $\left.\right|^{j, c}$ the effects of the tax reform which involves simultaneously cutting the $\operatorname{tax} j=F$ and increasing the tax $k=c$ so that as to leave balanced the government budget condition. In brief, the tax reform strategy involves simultaneously cutting the payroll tax by $\mathrm{d} \tau^{F}<0$ and increasing the tax on consumption goods $\left.\mathrm{d} \tau^{c}\right|^{F, c}>0$.

Holding $\tau^{H}$ constant, we differentiate (113)

$$
p_{c} \tilde{c}+\left.\tau^{c} p_{c} \mathrm{~d} \tilde{c}\right|^{F, c}+\left[\left(\tau^{F}+\tau^{H}\right) w_{\tau^{F}}+\tilde{w}\right] \mathrm{d} \tau^{F}+\left.\left(\tilde{w}^{F}-\tilde{w}^{A}\right) \mathrm{d} \tilde{L}\right|^{F, c}=0,
$$


with $\left[\left(\tau^{F}+\tau^{H}\right) w_{\tau^{F}}+\tilde{w}\right]=\tilde{w}\left(\frac{1-\tau^{H}}{1+\tau^{F}}\right)>0$.

By using the fact that $\left.\mathrm{d} \tilde{x}\right|^{F, c}=\frac{\mathrm{d} \tilde{x}}{\mathrm{~d} \tau^{F}} \mathrm{~d} \tau^{F}+\left.\frac{\mathrm{d} \tilde{x}}{\mathrm{~d} \tau^{c}} \mathrm{~d} \tau^{c}\right|^{F, c}$, and by rearranging terms, we can determine the size of the rise in the consumption tax rate $\left.\tau^{c}\right|^{F, c}$ after a fall in the payroll tax $\tau^{F}$ such that the governement budget constraint (113) remains balanced:

$$
\left.\mathrm{d} \tau^{c}\right|^{F, c}=-\frac{\chi_{F}}{\chi_{c}} \mathrm{~d} \tau^{H}=-\left\{\frac{\tau^{c} p_{c} \frac{\mathrm{d} \tilde{c}}{\mathrm{~d} \tau^{F}}+\left(\tilde{w}^{F}-\tilde{w}^{A}\right) \frac{\mathrm{d} \tilde{L}}{\mathrm{~d} \tau^{F}}+\left(\frac{1-\tau^{H}}{1+\tau^{F}}\right) \tilde{w} \tilde{L}}{\tau^{c} p_{c} \frac{\mathrm{d} \tilde{c}}{\mathrm{~d} \tau^{c}}+\left(\tilde{w}^{F}-\tilde{w}^{A}\right) \frac{\mathrm{d} \tilde{L}}{\mathrm{~d} \tau^{c}}+p_{c} \tilde{c}}\right\} \mathrm{d} \tau^{F}
$$

where the signs of $\chi_{F}$ and $\chi_{c}$ will be estimated later.

case $k^{N}>k^{T}$

If the non traded sector is relatively more capital intensive than the traded sector, the long-run changes are given by:

$$
\begin{aligned}
\left.\mathrm{d} \bar{\lambda}\right|^{F, c} & =\lambda_{\tau^{F}} \mathrm{~d} \tau^{F}+\left.\lambda_{\tau^{c}} \mathrm{~d} \tau^{c}\right|^{F, c} \\
& =\frac{\bar{\lambda}}{\chi_{c}} \frac{p_{c} \tilde{c}}{\Delta}\left\{\frac{\chi_{F}}{1+\tau^{c}} \sigma_{c}\left(1+\frac{\sigma_{L} \tilde{w}^{F} \tilde{L}}{\sigma_{c} p_{c} \tilde{c}}\right)+\sigma_{L} \tilde{w}^{F} \tilde{L} \frac{\tilde{\Lambda}}{1+\tau^{F}}\left(1-\frac{\tilde{w}^{A} \tilde{L}}{p_{c}\left(1+\tau^{c}\right) \tilde{c}}\right)\right\} \mathrm{d} \tau^{F} \\
& +\frac{\bar{\lambda}}{\chi_{c} \Delta} \frac{\omega_{2}^{1}}{\nu_{2}} \frac{r^{\star}}{\nu_{2}}\left(\sigma_{c} \tilde{c}^{N}-\sigma_{L} \tilde{L} k^{T} \nu_{2} \tilde{\Lambda}\right)\left[\frac{\chi_{F}}{1+\tau^{c}}\left(\sigma_{c} \tilde{c}^{N}-\sigma_{L} \tilde{L} k^{T} \nu_{2}\right)\right. \\
& \left.-\sigma_{L} \tilde{L} k^{T} \nu_{2} \frac{\tilde{\Lambda}}{1+\tau^{F}} p_{c} \tilde{c}\left(1-\frac{\tilde{w}^{A} \tilde{L}}{p_{c}\left(1+\tau^{c}\right) \tilde{c}}\right)\right] \mathrm{d} \tau^{F}<0 \\
\left.\mathrm{~d} \tilde{x}\right|^{F, c} & =\frac{\partial \tilde{x}}{\partial \tau^{F}} \mathrm{~d} \tau^{F}+\left.\frac{\partial \tilde{x}}{\partial \tau^{c}} \mathrm{~d} \tau^{c}\right|^{F, c} \\
& =\frac{\partial \tilde{x}}{\partial \tau^{F}} \frac{p_{c} \tilde{c}}{\chi_{c}}\left[1-\frac{\tilde{w}^{A} \tilde{L}}{p_{c} \tilde{c}\left(1+\tau^{c}\right)}\right] \mathrm{d} \tau^{F}>0
\end{aligned}
$$

where $x=c, K, L, b$. To determine (116b), we used the fact that $\frac{\partial \tilde{x}}{\partial \tau^{c}}=\frac{\partial \tilde{x}}{\partial \tau^{F}} \frac{1+\tau^{F}}{1+\tau^{c}} \frac{1}{\tilde{\Lambda}}$ and substituted (115), by remembering that $\chi_{c}=\frac{1+\tau^{F}}{1+\tau^{c}} \frac{1}{\tilde{\Lambda}}\left[\chi_{F}-\frac{\tilde{\Lambda}}{1+\tau^{F}} \tilde{w}^{A} \tilde{L}\right]+p_{c} \tilde{c}$.

We estimated $\chi_{c}$ and $\chi_{F}$ in the case $k^{N}>k^{T}$ as follows:

$$
\begin{aligned}
\chi_{c}= & \tau^{c} p_{c} \frac{\mathrm{d} \tilde{c}}{\mathrm{~d} \tau^{c}}+\left(\tilde{w}^{F}-\tilde{w}^{A}\right) \frac{\mathrm{d} \tilde{L}}{\mathrm{~d} \tau^{c}}+p_{c} \tilde{c} \\
= & \frac{\tilde{w}^{F} \tilde{L} p_{c} \tilde{c}}{\left(1+\tau^{c}\right) \Delta}\left\{\sigma_{c}\left(1+\tau^{c}\right)\left(\frac{p_{c} \tilde{c}}{\tilde{w}^{F} \tilde{L}}-\sigma_{L} \frac{\tau^{c}}{1+\tau^{c}}\right)+\sigma_{L}\left[\left(1+\tau^{c}\right)-\sigma_{c} \tau^{A}\right]\right\} \\
- & \left(1+\tau^{c}\right) \frac{\omega_{2}^{1}}{\nu_{2}} \frac{r^{\star}}{\nu_{2}}\left(\sigma_{c} \tilde{c}^{N}-\sigma_{L} \tilde{L} k^{T} \nu_{2} \tilde{\Lambda}\right) \\
& \left\{\sigma_{L} \tilde{L} k^{T} \nu_{2} \tilde{\Lambda}\left(1+\sigma_{L} \frac{\tau^{c}}{1+\tau^{c}}\right)-\sigma_{c} \tilde{c}^{N}\left(1+\sigma_{L} \frac{\tilde{w}^{F} \tilde{L}}{p_{c}\left(1+\tau^{c}\right) \tilde{c}} \tau^{A}\right)\right\}>0 \\
\chi_{F}= & \tau^{c} p_{c} \frac{\mathrm{d} \tilde{c}}{\mathrm{~d} \tau^{F}}+\left(\tilde{w}^{F}-\tilde{w}^{A}\right) \frac{\mathrm{d} \tilde{L}}{\mathrm{~d} \tau^{F}}+\left(\frac{\tilde{\Lambda}}{1+\tau^{F}}\right) \tilde{w}^{A} \tilde{L} .
\end{aligned}
$$

While the sign of $\chi_{c}$ is positive, we are unable to determine the sign of $\chi_{F}$. However, since it is reasonable to suppose that a rise in the consumption tax rate is required after a fall in the payroll tax rate for the government budget to be balanced, we assume that $\chi_{F}>0$. 
case $k^{T}>k^{N}$

If the traded sector is relatively more capital intensive than the non traded sector, the long-run changes are given by:

$$
\begin{aligned}
\left.\mathrm{d} \bar{\lambda}\right|^{F, c} & =\lambda_{\tau^{F}} \mathrm{~d} \tau^{F}+\left.\lambda_{\tau^{c}} \mathrm{~d} \tau^{c}\right|^{F, c}, \\
& =\frac{\lambda_{\tau^{F}}}{\chi_{c}}\left\{\frac{1+\tau^{F}}{1+\tau^{c}} \frac{\chi_{F}}{\tilde{\Lambda}}\left(1+\frac{\sigma_{c} p_{c} \tilde{c}}{\sigma_{L} \tilde{w}^{F} \tilde{L}}\right)+p_{c} \tilde{c}\left(1-\frac{\tilde{w}^{A} \tilde{L}}{p_{c} \tilde{c}\left(1+\tau^{c}\right)}\right)\right\} \mathrm{d} \tau^{F}<0, \\
\left.\mathrm{~d} \tilde{c}\right|^{F, c} & =\frac{\partial \tilde{x}}{\partial \tau^{F}} \mathrm{~d} \tau^{F}+\left.\frac{\partial \tilde{x}}{\partial \tau^{c}} \mathrm{~d} \tau^{c} \lambda_{\tau^{c}} \mathrm{~d} \tau^{c}\right|^{F, c} \\
& =\frac{\partial \tilde{x}}{\partial \tau^{F}} \frac{p_{c} \tilde{c}}{\chi_{c}}\left[1-\frac{\tilde{w}^{A} \tilde{L}}{p_{c} \tilde{c}\left(1+\tau^{c}\right)}\right] \mathrm{d} \tau^{F}>0,
\end{aligned}
$$

where $x=c, K, L, b$. To derive (118a), we made use of the fact that $\lambda_{\tau^{c}}=-\lambda_{\tau^{F}} \frac{\sigma_{c} p_{c} \tilde{c}}{\sigma_{L} \tilde{w}^{F} \tilde{L}} \frac{1}{\hat{\Lambda}} \frac{1+\tau^{F}}{1+\tau^{c}}$. To determine (118b), we used the fact that $\frac{\partial \tilde{x}}{\partial \tau^{c}}=\frac{\partial \tilde{x}}{\partial \tau^{F}} \frac{1+\tau^{F}}{1+\tau^{c}} \frac{1}{\tilde{\Lambda}}$ and substituted (115), by remembering that $\chi_{c}=\frac{1+\tau^{F}}{1+\tau^{c}} \frac{1}{\tilde{\Lambda}}\left[\chi_{F}-\frac{\tilde{\Lambda}}{1+\tau^{F}} \tilde{w}^{A} \tilde{L}\right]+p_{c} \tilde{c}$. It is interesting to notice that the fall in the marginal utility of wealth following a rise in $\tau^{c}$ is strengthened by its decrease after a reduction in $\tau^{F}$.

We estimated $\chi_{c}$ and $\chi_{F}$ in the case $k^{T}>k^{N}$ as follows:

$$
\begin{aligned}
\chi_{c} & =\tau^{c} p_{c} \frac{\mathrm{d} \tilde{c}}{\mathrm{~d} \tau^{c}}+\left(\tilde{w}^{F}-\tilde{w}^{A}\right) \frac{\mathrm{d} \tilde{L}}{\mathrm{~d} \tau^{c}}+p_{c} \tilde{c} \\
& =\frac{\tilde{w}^{F} \tilde{L} p_{c} \tilde{c}}{\left(1+\tau^{c}\right) \Delta}\left\{\sigma_{c}\left(1+\tau^{c}\right)\left(\frac{p_{c} \tilde{c}}{\tilde{w}^{F} \tilde{L}}-\sigma_{L} \frac{\tau^{c}}{1+\tau^{c}}\right)+\sigma_{L}\left[\left(1+\tau^{c}\right)-\sigma_{c} \tau^{A}\right]\right\}>0 \\
\chi_{F} & =\tau^{c} p_{c} \frac{\mathrm{d} \tilde{c}}{\mathrm{~d} \tau^{F}}+\left(\tilde{w}^{F}-\tilde{w}^{A}\right) \frac{\mathrm{d} \tilde{L}}{\mathrm{~d} \tau^{F}}+\left(\frac{1-\tau^{H}}{1+\tau^{F}}\right) \tilde{w} \tilde{L} \\
& =\frac{\tilde{w}^{F} \tilde{L} p_{c} \tilde{c}}{\Delta} \frac{\tilde{\Lambda}}{1+\tau^{F}}\left\{\sigma_{c}\left(\frac{\tilde{w}^{A}}{\tilde{w}^{F}}-\sigma_{c} \tau^{c}\right)+\sigma_{L}\left(\frac{\tilde{w}^{A} \tilde{L}}{p_{c} \tilde{c}}-\sigma_{c} \tau^{A}\right)\right\} \gtrless 0
\end{aligned}
$$

The term $\chi_{c}$ captures two conflictory effects induced by a rise in the consumption tax rate on public revenue. For given levels of real consumption and employment, the increased consumption tax rate raises fiscal earnings as it is reflected by the third term on the RHS of (119a) (first line). However, by increasing the consumption tax rate, households are induced to reduce both their real consumption and their labor supply. Inspection of (119a) (second line) shows that the first term in braces is positive since $\frac{p_{c} \tilde{c}}{\tilde{w}^{F} \tilde{L}}>1$ as longer as $\tilde{a}>0$ and because $\frac{\tau^{c}}{1+\tau^{c}}$ is very small (the average value of the consumption tax rate for 13 OECD countries is approximately 10\%). The second term of (119a) (second line) is also positive since $\sigma_{c}$ is lower than unity according to empirical evidence and $0<\tau^{A}<1$. Consequently, according to (119a), $\chi_{c}>0$; in brief, the decrease in the tax bases via lower consumption and employment is not large enough to more than outweigh the positive tax rate effect.

The sign of $\chi_{F}$ is unclear. Inspection of (119c) shows that the signs of the two terms in braces are ambiguous: [i] because $\frac{\tilde{w}^{A}}{\tilde{w}^{F}}<1$ and $\sigma_{L} \tau^{c}<1$, and since [ii] $\frac{\tilde{w}^{A} \tilde{L}}{p_{c} \tilde{c}}<1$ and $\sigma_{c} \tau^{A}<1$. However, we may reasonably expect that a fall in the payroll tax, i. e. $\mathrm{d} \tau^{F}<0$, does not pay for itself so that the rise in the tax bases via higher consumption and higher employment, is not large enough to offset the negative public revenue effect originating from 
the cut of the labor income tax rate $\mathrm{d} \tau^{F}$. Consequently, we may assume that $\chi_{F}>0$ so that the consumption tax rate must rise for the government balanced budget to hold.

\section{F.2 Steady-State Changes of a Tax Reform: Substitution of labor income Taxes for Consumption Taxes}

In this section, we estimate the long-run effects of a fall in the labor income $\operatorname{tax} \tau^{H}$ associated with a rise in the consumption tax rate $\tau^{c}$, which is adjusted accordingly to balance the government budget. Additionally, we assume that taxes on labor income are progressive so that $\kappa>0$ and $\Lambda<1$. To avoid confusion, we denote by $\left.\right|^{j, c}$ the effects of the tax reform which involves simultaneously cutting the tax $j=H$ and increasing the tax $k=c$ so that as to to leave balanced the government budget condition. In brief, the tax reform strategy involves simultaneously cutting the labor income tax by $\mathrm{d} \tau^{H}<0$ and increasing the tax on consumption goods $\left.\mathrm{d} \tau^{c}\right|^{F, c}>0$.

Differentiate (113) by letting $\tau^{F}$ constant, we can derive the size of the rise in the consumption tax rate:

$$
\left.\mathrm{d} \tau^{c}\right|^{H, c}=-\frac{\chi_{H}}{\chi_{c}} \mathrm{~d} \tau^{H}=-\left\{\frac{\tau^{c} p_{c} \frac{\mathrm{d} \tilde{c}}{\mathrm{~d} \tau^{H}}+\left(\tilde{w}^{F}-\tilde{w}^{A}\right) \frac{\mathrm{d} \tilde{L}}{\mathrm{~d} \tau^{H}}+(\tilde{w}-\kappa) \tilde{L}}{\tau^{c} p_{c} \frac{\mathrm{d} \tilde{c}}{\mathrm{~d} \tau^{c}}+\left(\tilde{w}^{F}-\tilde{w}^{A}\right) \frac{\mathrm{d} \tilde{L}}{\mathrm{~d} \tau^{c}}+p_{c} \tilde{c}}\right\} \mathrm{d} \tau^{H}
$$

Making use of long-term effects of permanent changes in $\tau^{H}$ and $\tau^{c}$ and substituting $\left.\mathrm{d} \tau^{c}\right|^{H, c}$ given by (120), we are able to estimate the directions and the sizes of the long-run changes of main economic variables after a fall in $\tau^{H}$ associated with a rise in $\tau^{c}$ by an amount that leaves balanced the government budget constraint. As it is formally shown below, long-run changes are not qualitatively sensitive to sectoral capital-labor ratios. Instead, their magnitude depends on the steady-state variations after a fall in $\tau^{F}$. Hence, the beneficial effects of a fiscal reform will be sensitive to sectoral capital intensities due to the feed-back effect or "secondary effect" originating from the real exchange rate dynamics over the transition.

$$
\text { case } k^{N}>k^{T}
$$

If the non traded sector is relatively more capital intensive than the traded sector, the 
long-run changes are given by:

$$
\begin{aligned}
\left.\mathrm{d} \bar{\lambda}\right|^{H, c} & =\lambda_{\tau^{H}} \mathrm{~d} \tau^{H}+\left.\lambda_{\tau^{c}} \mathrm{~d} \tau^{c}\right|^{H, c}, \\
& =\frac{\bar{\lambda}}{\chi_{c}} \frac{p_{c} \tilde{c}}{\Delta}\left\{\frac{\chi_{H}}{1+\tau^{c}} \sigma_{c}\left(1+\frac{\sigma_{L} \tilde{w}^{F} \tilde{L}}{\sigma_{c} p_{c} \tilde{c}}\right)+\sigma_{L} \tilde{w}^{F} \tilde{L}\left(\frac{\tilde{w}-\kappa}{\tilde{w}}\right)\left(1-\frac{\tilde{w}^{A} \tilde{L}}{p_{c}\left(1+\tau^{c}\right) \tilde{c}}\right)\right\} \mathrm{d} \tau^{H} \\
& +\frac{\bar{\lambda}}{\chi_{c} \Delta} \frac{\omega_{2}^{1}}{\nu_{2}} \frac{r^{\star}}{\nu_{2}}\left(\sigma_{c} \tilde{c}^{N}-\sigma_{L} \tilde{L} k^{T} \nu_{2} \tilde{\Lambda}\right)\left[\frac{\chi_{H}}{1+\tau^{c}}\left(\sigma_{c} \tilde{c}^{N}-\sigma_{L} \tilde{L} k^{T} \nu_{2}\right)\right. \\
& \left.-\sigma_{L} \tilde{L} k^{T} \nu_{2}\left(\frac{\tilde{w}-\kappa}{\tilde{w}}\right) p_{c} \tilde{c}\left(1-\frac{\tilde{w}^{A} \tilde{L}}{p_{c}\left(1+\tau^{c}\right) \tilde{c}}\right)\right] \mathrm{d} \tau^{H}>0, \\
\left.\mathrm{~d} \tilde{x}\right|^{H, c} & =\frac{\partial \tilde{x}}{\partial \tau^{H}} \mathrm{~d} \tau^{H}+\left.\frac{\partial \tilde{x}}{\partial \tau^{c}} \mathrm{~d} \tau^{c}\right|^{H, c} \\
& =\frac{\partial \tilde{x}}{\partial \tau^{H}} \frac{p_{c} \tilde{c}}{\chi_{c}}\left[1-\frac{\tilde{w}^{A} \tilde{L}}{p_{c} \tilde{c}\left(1+\tau^{c}\right)}\right] \mathrm{d} \tau^{H},
\end{aligned}
$$

where $x=c, K, L, b$. To determine $(121 \mathrm{~b})$, we used the fact that $\frac{\partial \tilde{x}}{\partial \tau^{c}}=\frac{\partial \tilde{x}}{\partial \tau^{H}} \frac{1}{1+\tau^{c}}\left(\frac{\tilde{w}^{A}}{\tilde{w}-\kappa}\right)$ and substituted (120), by remembering that $\chi_{c}=\frac{1}{1+\tau^{c}}\left(\frac{\tilde{w}^{A}}{\tilde{w}-\kappa}\right)\left[\chi_{H}-(\tilde{w}-\kappa) \tilde{L}\right]+p_{c} \tilde{c}$.

We estimated $\chi_{c}$ and $\chi_{H}$ in the case $k^{N}>k^{T}$ as follows:

$$
\begin{aligned}
\chi_{c}= & \tau^{c} p_{c} \frac{\mathrm{d} \tilde{c}}{\mathrm{~d} \tau^{c}}+\left(\tilde{w}^{F}-\tilde{w}^{A}\right) \frac{\mathrm{d} \tilde{L}}{\mathrm{~d} \tau^{c}}+p_{c} \tilde{c} \\
= & \frac{\tilde{w}^{F} \tilde{L} p_{c} \tilde{c}}{\left(1+\tau^{c}\right) \Delta}\left\{\sigma_{c}\left(1+\tau^{c}\right)\left(\frac{p_{c} \tilde{c}}{\tilde{w}^{F} \tilde{L}}-\sigma_{L} \frac{\tau^{c}}{1+\tau^{c}}\right)+\sigma_{L}\left[\left(1+\tau^{c}\right)-\sigma_{c} \tau^{A}\right]\right\} \\
+ & \left(1+\tau^{c}\right) \frac{\omega_{2}^{1}}{\nu_{2}} \frac{r^{\star}}{\nu_{2}}\left(\sigma_{c} \tilde{c}^{N}-\sigma_{L} \tilde{L} k^{T} \nu_{2} \tilde{\Lambda}\right) \\
& \left\{-\sigma_{L} \tilde{L} k^{T} \nu_{2} \tilde{\Lambda}\left(1+\sigma_{L} \frac{\tau^{c}}{1+\tau^{c}}\right)+\sigma_{c} \tilde{c}^{N}\left(1+\sigma_{L} \frac{\tilde{w}^{F} \tilde{L}}{p_{c}\left(1+\tau^{c}\right) \tilde{c}} \tau^{A}\right)\right\}>0,(12) \\
\chi_{H}= & \tau^{c} p_{c} \frac{\mathrm{d} \tilde{c}}{\mathrm{~d} \tau^{H}}+\left(\tilde{w}^{F}-\tilde{w}^{A}\right) \frac{\mathrm{d} \tilde{L}}{\mathrm{~d} \tau^{H}}+(\tilde{w}-\kappa) \tilde{L}>0
\end{aligned}
$$

While the sign of $\chi_{c}$ is positive, we are unable to determine the sign of $\chi_{H}$. However, since it is reasonable to suppose that a rise in the consumption tax rate is required after a fall in the labor income tax rate for the government budget to be balanced, we assume that $\chi_{H}>0$.

$$
\text { case } k^{T}>k^{N}
$$

If the traded sector is relatively more capital intensive than the non traded sector, the long-run changes are given by:

$$
\begin{aligned}
\left.\mathrm{d} \bar{\lambda}\right|^{H, c} & =\lambda_{\tau^{H}} \mathrm{~d} \tau^{H}+\left.\lambda_{\tau^{c}} \mathrm{~d} \tau^{c}\right|^{H, c} \\
& =\frac{\lambda_{\tau^{H}}}{\chi_{c}}\left\{\frac{\chi_{H}}{1+\tau^{c}}\left(\frac{\tilde{w}^{A}}{\tilde{w}-\kappa}\right)\left(1+\frac{\sigma_{c} p_{c} \tilde{c}}{\sigma_{L} \tilde{w}^{F} \tilde{L}}\right)+p_{c} \tilde{c}\left(1-\frac{\tilde{w}^{A} \tilde{L}}{p_{c} \tilde{c}\left(1+\tau^{c}\right)}\right)\right\} \mathrm{d} \tau^{H} \\
\left.\mathrm{~d} \tilde{x}\right|^{H, c} & =\frac{\partial \tilde{x}}{\partial \tau^{H}} \mathrm{~d} \tau^{H}+\left.\frac{\partial \tilde{x}}{\partial \tau^{c}} \mathrm{~d} \tau^{c}\right|^{H, c} \\
& =\frac{\partial \tilde{x}}{\partial \tau^{H}} \frac{p_{c} \tilde{c}}{\chi_{c}}\left[1-\frac{\tilde{w}^{A} \tilde{L}}{p_{c} \tilde{c}\left(1+\tau^{c}\right)}\right] \mathrm{d} \tau^{H}
\end{aligned}
$$


where $x=c, K, L, b$. To derive (123a), we made use of the fact that $\lambda_{\tau^{c}}=-\lambda_{\tau^{H}} \frac{\sigma_{c} p_{c} \tilde{c}}{\sigma_{L} \tilde{w}^{F} \tilde{L}} \frac{\tilde{w}^{A}}{(\tilde{w}-\kappa)}$. To determine (123b), we used the fact that $\frac{\partial \tilde{x}}{\partial \tau^{c}}=\frac{\partial \tilde{x}}{\partial \tau^{H}} \frac{1}{1+\tau^{c}}\left(\frac{\tilde{w}^{A}}{\tilde{w}-\kappa}\right)$ and substituted (120), by remembering that $\chi_{c}=\frac{1}{1+\tau^{c}}\left(\frac{\tilde{w}^{A}}{\tilde{w}-\kappa}\right)\left[\chi_{H}-(\tilde{w}-\kappa) \tilde{L}\right]+p_{c} \tilde{c}$. It is interesting to notice that the fall in the marginal utility of wealth following a rise in $\tau^{c}$ is strengthened by its decrease after a reduction in $\tau^{H}$.

We estimated $\chi_{c}$ and $\chi_{H}$ in the case $k^{T}>k^{N}$ as follows:

$$
\begin{aligned}
\chi_{c} & =\tau^{c} p_{c} \frac{\mathrm{d} \tilde{c}}{\mathrm{~d} \tau^{c}}+\left(\tilde{w}^{F}-\tilde{w}^{A}\right) \frac{\mathrm{d} \tilde{L}}{\mathrm{~d} \tau^{c}}+p_{c} \tilde{c} \\
& =\frac{\tilde{w}^{F} \tilde{L} p_{c} \tilde{c}}{\left(1+\tau^{c}\right) \Delta}\left\{\sigma_{c}\left(1+\tau^{c}\right)\left(\frac{p_{c} \tilde{c}}{\tilde{w}^{F} \tilde{L}}-\sigma_{L} \frac{\tau^{c}}{1+\tau^{c}}\right)+\sigma_{L}\left[\left(1+\tau^{c}\right)-\sigma_{c} \tau^{A}\right]\right\}>0, \\
\chi_{H} & =\tau^{c} p_{c} \frac{\mathrm{d} \tilde{c}}{\mathrm{~d} \tau^{H}}+\left(\tilde{w}^{F}-\tilde{w}^{A}\right) \frac{\mathrm{d} \tilde{L}}{\mathrm{~d} \tau^{H}}+(\tilde{w}-\kappa) \tilde{L} \\
& =\frac{\tilde{w}^{F} \tilde{L} p_{c} \tilde{c}}{\Delta}\left(\frac{\tilde{w}-\kappa}{\tilde{w}^{A}}\right)\left\{\sigma_{c}\left(\frac{\tilde{w}^{A}}{\tilde{w}^{F}}-\sigma_{L} \tau^{c}\right)+\sigma_{L}\left(\frac{\tilde{w}^{A} \tilde{L}}{p_{c} \tilde{c}}-\sigma_{c} \tau^{A}\right)\right\} \gtrless 0,
\end{aligned}
$$

The term $\chi_{c}$ captures two conflictory effects induced by a rise in the consumption tax rate on public revenue. For given levels of real consumption and employment, the increased consumption tax rate raises fiscal earnings as it is reflected by the third term on the RHS of (124a) (first line). However, by increasing the consumption tax rate, households are induced to reduce both their real consumption and their labor supply. Inspection of (124a) (second line) shows that the first term in braces is positive since $\frac{p_{c} \tilde{c}}{\tilde{w}^{F} \tilde{L}}>1$ as longer as $\tilde{a}>0$ and because $\frac{\tau^{c}}{1+\tau^{c}}$ is very small (the average value of the consumption tax rate for 13 OECD countries is approximately 10\%). The second term of (124a) (second line) is also positive since $\sigma_{c}$ is lower than unity according to empirical evidence and $0<\tau^{A}<1$. Consequently, according to (124a), $\chi_{c}>0$; in brief, the decrease in the tax bases via lower consumption and employment is not large enough to more than outweigh the positive tax rate effect.

The sign of $\chi_{H}$ is unclear. Inspection of (124b) shows that the signs of the two terms in braces are ambiguous: [i] because $\frac{\tilde{w}^{A}}{\tilde{w}^{F}}<1$ and $\sigma_{L} \tau^{c}<1$, and since [ii] $\frac{\tilde{w}^{A} \tilde{L}}{p_{c} \tilde{c}}<1$ and $\sigma_{c} \tau^{A}<1$. However, we may reasonably expect that a fall in the labor income tax rate $\mathrm{d} \tau^{H}<0$ does not pay itself so that the rise in the tax bases via higher consumption and higher employment, is not large enough to offset the negative public revenue effect originating from the cut of the labor income tax rate $\mathrm{d} \tau^{H}$. Consequently, we may assume that $\chi_{H}>0$ so that the consumption tax rate must rise for the government balanced budget to hold.

\section{G Dynamic Effects of a Tax Reform}

This section estimates the dynamic effects of a tax restructuring. Steady-state changes are those derived into the previous section where we estimated the long-run variations such that the rise in $\tau^{c}$ guarantees that the balanced condition for the government holds. In addition, we consider that the change of the tax scheme can be viewed as an unanticipated permanent tax shock, i. e. in the two first tax reforms we considered, the labor and the consumption tax 
rates are changed simultaneously so as the government budget balanced condition is met and in the third tax reform, the payroll and the labor income tax rates are changes simultaneously so as to leave unchanged the marginal tax wedge.

The stable adjustment of the economy is described by a saddle-path in $(K, p)$-space. The capital stock, the real exchange rate, and the stock of traded bonds evolve according to:

$$
\begin{aligned}
K(t) & =\tilde{K}+B_{1} e^{\nu_{1} t}, \\
p(t) & =\tilde{p}+\omega_{2}^{1} B_{1} e^{\nu_{1} t} \\
b(t) & =\tilde{b}+\Phi_{1} B_{1} e^{\nu_{1} t}
\end{aligned}
$$

where $\omega_{2}^{1}=0$ if $k^{T}>k^{N}$ and with

$$
B_{1}=K_{0}-\tilde{K}=-\left.\mathrm{d} \tilde{K}\right|^{j, k},
$$

where we made use of the constancy of $K$ at time $t=0$ (i. e. $K_{0}$ is predetermined).

\section{G.1 A Permanent Rise in the Consumption Tax Rate}

case $k^{N}>k^{T}$

Using the fact that the steady-state value of the real exchange rate remains unaffected by the change of the consumption tax rate, the initial reaction of the relative price of non tradables is given by:

$$
\frac{\mathrm{d} p(0)}{\mathrm{d} \tau^{c}}=-\omega_{2}^{1} \mathrm{~d} \frac{\mathrm{d} \tilde{K}}{\mathrm{~d} \tau^{c}}<0,
$$

where $\omega_{2}^{1}<0$ and the long-run change of the capital stock is given by (95e).

Regarding the initial reaction of real consumption, evaluating first at time $t=0$ and differentiating the short-run static solution for real consumption (44) w. r. t. $\tau^{c}$ leads to:

$$
\begin{aligned}
\frac{\mathrm{d} c(0)}{\mathrm{d} \tau^{c}} & =c_{\bar{\lambda}} \frac{\mathrm{d} \bar{\lambda}}{\mathrm{d} \tau^{c}}+c_{p} \frac{\mathrm{d} p(0)}{\mathrm{d} \tau^{c}}+c_{\tau^{c}}, \\
& =-\frac{\sigma_{c} \tilde{c} \sigma_{L} \tilde{L}}{\Delta\left(1+\tau^{c}\right)}\left\{\tilde{w}^{F}\left(1+\alpha_{c} \sigma_{c} \frac{\tilde{c}^{N}}{\tilde{p}} \frac{\omega_{2}^{1}}{\nu_{2}}\right)-\tilde{k}^{T} \frac{\omega_{2}^{1}}{\nu_{2}}\left(\sigma_{c} \tilde{c}^{N} \nu_{1}-r^{\star} \sigma_{L} \tilde{L} \tilde{k}^{T} \nu_{2}\right)\right\} \lessgtr 0 .
\end{aligned}
$$

Regarding the initial reaction of employment, evaluating first at time $t=0$ and differentiating the short-run static solution for labor supply $(44)$ w. r. t. $\tau^{c}$ leads to:

$$
\frac{\mathrm{d} L(0)}{\mathrm{d} \tau^{c}}=L_{\bar{\lambda}} \frac{\mathrm{d} \bar{\lambda}}{\mathrm{d} \tau^{c}}+L_{p} \frac{\mathrm{d} p(0)}{\mathrm{d} \tau^{c}} \lessgtr 0,
$$

with $L_{p}=-\sigma_{L} \frac{\tilde{L}}{\tilde{w}} \frac{\tilde{k}^{T}}{\left(1+\tau^{F}\right)} \nu_{2}<0$.

Differentiating solutions (125) with respect to time, we are able to compute the directions 
of trajectories:

$$
\begin{aligned}
\dot{K}(t) & =I(t)=-\nu_{1} \frac{\mathrm{d} \tilde{K}}{\mathrm{~d} \tau^{c}} e^{\nu_{1} t} \mathrm{~d} \tau^{c}<0, \\
\dot{p}(t) & =-\nu_{1} \omega_{2}^{1} \frac{\mathrm{d} \tilde{K}}{\mathrm{~d} \tau^{c}} e^{\nu_{1} t} \mathrm{~d} \tau^{c}=\omega_{2}^{1} I(t)>0, \\
\dot{b}(t) & =-\nu_{1} \Phi_{1} \frac{\mathrm{d} \tilde{K}}{\mathrm{~d} \tau^{c}} e^{\nu_{1} t} \mathrm{~d} \tau^{c}=\Phi_{1} I(t)>0,
\end{aligned}
$$

with $\Phi_{1}<0$ and $\frac{\mathrm{d} \tilde{K}}{\mathrm{~d} \tau^{c}}<0$.

$$
\text { case } k^{T}>k^{N}
$$

With the reversal of capital intensities, both real consumption and total employment fall immediately to their new lower long-run levels. Over the transition, the negative investment flow is exactly matched by a current account surplus, thus leaving unchanged savings.

\section{G.2 A Permanent Fall in the Payroll Tax Rate}

case $k^{N}>k^{T}$

Regarding the initial reaction of real consumption, evaluating first at time $t=0$ and differentiating the short-run static solution for real consumption (44) w. r. t. $\tau^{F}$ leads to:

$$
\begin{aligned}
\frac{\mathrm{d} c(0)}{\mathrm{d} \tau^{F}} & =c_{\bar{\lambda}} \frac{\mathrm{d} \bar{\lambda}}{\mathrm{d} \tau^{F}}+c_{p} \frac{\mathrm{d} p(0)}{\mathrm{d} \tau^{F}} \\
& =-\frac{\sigma_{c} \tilde{c} \sigma_{L} \tilde{L}}{\Delta\left(1+\tau^{F}\right)}\left\{\tilde{w}^{F}\left(1+\alpha_{c} \sigma_{c} \frac{\tilde{c}^{N}}{\tilde{p}} \frac{\omega_{2}^{1}}{\nu_{2}}\right)-\tilde{k}^{T} \frac{\omega_{2}^{1}}{\nu_{2}}\left(\sigma_{c} \tilde{c}^{N} \nu_{1}-r^{\star} \sigma_{L} \tilde{L} \tilde{k}^{T} \nu_{2}\right)\right\} \lessgtr(\mathbf{0 2} 9)
\end{aligned}
$$

Regarding the initial reaction of employment, evaluating first at time $t=0$ and differentiating the short-run static solution for labor supply (44) w. r. t. $\tau^{F}$ leads to:

$$
\frac{\mathrm{d} L(0)}{\mathrm{d} \tau^{F}}=L_{\bar{\lambda}} \frac{\mathrm{d} \bar{\lambda}}{\mathrm{d} \tau^{F}}+L_{p} \frac{\mathrm{d} p(0)}{\mathrm{d} \tau^{F}}+L_{\tau^{F}} \lessgtr 0 .
$$

case $k^{T}>k^{N}$

With the reversal of capital intensities, both real consumption and total employment reach immediately to their new higher long-run levels. Over the transition, the positive investment flow is exactly matched by a current account deficit, thus leaving unchanged savings.

\section{G.3 Dynamic Effects of a Tax Restructuring}

First, it is convenient to introduce some notations. We index by the superscript $j$ the impact and steady-state effects induced by a fiscal reform strategy which involves simultaneous cutting the labor tax $\mathrm{d} \tau^{j}$ (i e. $j=F$ if the payroll tax is reduced or $j=H$ if the labor income tax is lowered) and increasing the tax rate by $\mathrm{d} \tau^{k}(k=c, H)$. While the two first tax reform 
strategies involves a rise in the consumption tax rate by $\left.\mathrm{d} \tau^{c}\right|^{j, c}>0$ (so as to leave balanced the government budget constraint), the third tax reform strategy involves an increase in the labor income tax rate by $\left.\mathrm{d} t a u^{H}\right|^{F, H}>0$ (so as to leave unchanged the marginal tax wedge $\left.\tau^{M}\right)$.

This leads to long-run changes which can be written as follows:

$$
\left.\mathrm{d} \tilde{x}\right|^{j, k}=\frac{\mathrm{d} \tilde{x}}{\mathrm{~d} \tau^{j}} \Phi^{j, k} \mathrm{~d} \tau^{j}, \quad j=F, H, \quad k=c, H,
$$

where $x=c, L, K$ and we denoted by $0<\Phi^{j, k}<1$ the scaled-down term of the long-run change after a fall in the labor tax $(j=F, H)$. In addition, we denote by $\Upsilon^{j}$ the positive term:

$$
\Upsilon^{F}=\frac{\tilde{\Lambda}}{1+\tau^{F}}>0, \quad \Upsilon^{H}=\tilde{w}-\kappa>0,
$$

with $\Upsilon^{F}=\frac{1}{1+\tau^{F}}$ and $\Upsilon^{H}=1$ if $\kappa=0$.

$$
\text { case } k^{N}>k^{T}
$$

Using the fact that the steady-state value of the real exchange rate remains unaffected by a tax restructuring, the initial jump of $p$ is formally given by:

$$
\left.\mathrm{d} p(0)\right|^{j, k}=-\left.\omega_{2}^{1} \mathrm{~d} \tilde{K}\right|^{j, k}>0,
$$

where the long-run change of the capital stock is given by (131) ( short-run static solution for real consumption (44), the substitution of its long-run change (131) (set $x=c$ ) together with the initial jump of the real exchange rate (133), the initial reaction of real consumption is given by:

$$
\begin{aligned}
\left.\mathrm{d} c(0)\right|^{j, k} & =\left.\mathrm{d} \tilde{c}\right|^{j, k}+\left.c_{p} \mathrm{~d} p(0)\right|^{j, k}=\left[\frac{\mathrm{d} \tilde{c}}{\mathrm{~d} \tau^{j}}-c_{p} \omega_{2}^{1} \frac{\mathrm{d} \tilde{K}}{\mathrm{~d} \tau^{j}}\right] \Phi^{j, k} \mathrm{~d} \tau^{j}, \\
& =-\frac{\sigma_{c} \tilde{c} \sigma_{L} \tilde{L}}{\Delta} \Upsilon^{j}\left\{\tilde{w}^{F}\left(1+\alpha_{c} \sigma_{c} \frac{\tilde{c}^{N}}{\tilde{p}} \frac{\omega_{2}^{1}}{\nu_{2}}\right)\right. \\
& \left.-r^{\star} \tilde{k}^{T} \frac{\omega_{2}^{1}}{\nu_{2}}\left(\sigma_{c} \tilde{c}^{N} \nu_{1}-\sigma_{L} \tilde{L} \tilde{k}^{T} \nu_{2}\right)\right\} \Phi^{j, k} \mathrm{~d} \tau^{j} \gtrless 0,
\end{aligned}
$$

where we used the fact that $c_{p}=-\sigma_{c} \alpha_{c} \frac{\tilde{c}}{\tilde{p}}<0, \Upsilon^{j}>0$ (see (132)), $\left(1+\alpha_{c} \sigma_{c} \frac{\tilde{c}^{N}}{\tilde{p}} \frac{\omega_{2}^{1}}{\nu_{2}}\right)>0$ (see (87) by setting $\sigma_{L}=0$ ) and $\mathrm{d} \tau^{j}<0$ since we considered a fiscal reform strategy which simultaneously involves cutting labor tax and raising the consumption tax rate so as to leave balanced the government budget constraint; in addition, $\Delta>0$.

By applying a similar procedure to labor, we can derive its reaction once the fiscal reform is implemented, i. e. at time $t=0$ :

$$
\begin{aligned}
\left.\mathrm{d} L(0)\right|^{j, k} & =\left.\mathrm{d} \tilde{L}\right|^{j, k}+\left.L_{p} \mathrm{~d} p(0)\right|^{j, k}=\left[\frac{\mathrm{d} \tilde{L}}{\mathrm{~d} \tau^{j}}-L_{p} \omega_{2}^{1} \frac{\mathrm{d} \tilde{K}}{\mathrm{~d} \tau^{j}}\right] \Phi^{j, k} \mathrm{~d} \tau^{j}, \\
& =-\frac{\sigma_{L} \tilde{L}}{\Delta} \Upsilon^{j}\left\{\sigma_{c} p_{c} \tilde{c}+\frac{\omega_{2}^{1}}{\nu_{2}}\left[\sigma_{L} \tilde{L} \tilde{\Lambda} \tilde{k}^{T} \nu_{2} \sigma_{c}\left(\tilde{c}^{N}+\frac{p_{c} \tilde{c}}{\tilde{w}^{F} \tilde{L}} \tilde{L} \tilde{k}^{T} \nu_{2}\right)\right.\right. \\
& \left.\left.+\frac{r^{\star}}{\nu_{2}}\left(\sigma_{c} \tilde{c}^{N}-\sigma_{L} \tilde{L} \tilde{k}^{T} \nu_{2} \tilde{\Lambda}\right) \sigma_{c} \tilde{c}^{N}\right]\right\} \Phi^{j, k} \mathrm{~d} \tau^{j} \gtrless 0,
\end{aligned}
$$


where $\Delta>0, \mathrm{~d} \tau^{j}<0$, and we used the fact that $L_{p}=-\frac{\sigma_{L} \tilde{L} \tilde{\Lambda}^{T} \tilde{k}^{T} \nu_{2}}{\tilde{w}^{F}}<0, \Upsilon^{j}>0$ (see (132)).

Differentiating solutions (125) with respect to time, we are able to compute the directions of trajectories:

$$
\begin{aligned}
\dot{K}(t) & =I(t)=-\left.\nu_{1} \mathrm{~d} \tilde{K}\right|^{j, k} e^{\nu_{1} t}>0, \\
\dot{p}(t) & =-\left.\nu_{1} \omega_{2}^{1} \mathrm{~d} \tilde{K}\right|^{j, k} e^{\nu_{1} t}=\omega_{2}^{1} I(t)<0, \\
\dot{b}(t) & =-\left.\nu_{1} \Phi_{1} \mathrm{~d} \tilde{K}\right|^{j, k} e^{\nu_{1} t}=\Phi_{1} I(t)<0,
\end{aligned}
$$

with $\Phi_{1}<0$ and $\left.\mathrm{d} \tilde{K}\right|^{j, k}>0$.

Regarding consumption and labor supply behavior, their trajectories can be computed by linearizing their short-run static solutions in the neighborhood of the steady-state and differentiating these with respect to time:

$$
\dot{c}(t)=c_{p} \dot{p}(t)>0, \quad \dot{L}(t)=L_{p} \dot{p}(t)>0,
$$

with $c_{p}<0$ and $L_{p}<0$. From impact and steady-state effects, their rising temporal profiles imply that if they rise in the short-run, their initial upward jumps display a smaller size than the size of their long-run changes.

case $k^{T}>k^{N}$

Since the real exchange rate remains unaffected by the fiscal shock in the short-run, real consumption and labor jump immediately to their new higher steady-state levels.

Differentiating solutions (125) with respect to time, we are able to compute the directions of trajectories:

$$
\begin{aligned}
\dot{K}(t) & =I(t)=-\left.\nu_{1} \mathrm{~d} \tilde{K}\right|^{j, k} e^{\nu_{1} t}>0, \\
\dot{p}(t) & =0, \\
\dot{b}(t) & =-\left.\nu_{1} \Phi_{1} \mathrm{~d} \tilde{K}\right|^{j, k} e^{\nu_{1} t}=\Phi_{1} I(t)<0 .
\end{aligned}
$$

\section{H Tax Wedge}

In line with general practice, payroll taxes are assumed to be proportional and wage income taxes are taken to be progressive. Following Heijdra and Lightart [2008], we define the average tax wedge as the difference between the producer wage (paid by the firm) and the purchasing power on consumption goods of after-tax average wage expressed as a percentage of the wage including payroll taxes:

$$
\begin{aligned}
\tau^{A} & \equiv \frac{w L\left(1+\tau^{F}\right)-\left[\left(1-\tau^{H}\right) w+\tau^{H} \kappa\right] L}{w^{F} L}, \\
& \equiv 1-\frac{\left[\left(1-\tau^{H}\right)+\frac{\tau^{H} \kappa}{w}\right]}{\left(1+\tau^{F}\right)}
\end{aligned}
$$


where $w^{F}=w\left(1+\tau^{F}\right)$. In addition, we denote by $\tau^{M}$ the marginal tax wedge as the difference between the producer wage (paid by the firm) and the after-tax marginal wage expressed as a percentage of the producer cost (i. e. including payroll taxes):

$$
\begin{aligned}
\tau^{M} & \equiv \frac{w L\left(1+\tau^{F}\right)-w L\left(1-\tau^{H}\right)}{w^{F} L}, \\
& \equiv 1-\frac{\left(1-\tau^{H}\right)}{\left(1+\tau^{F}\right)}
\end{aligned}
$$

The closer to unity $\tau^{M}$, the larger the gap between the wage paid by firms and the real wage received by households.

Using the definition of $\tau^{M}$ given by (140), we can rewrite the average tax wedge as follows:

$$
\tau^{A} \equiv \tau^{M}-\frac{\tau^{H} \kappa}{w^{F}}
$$

Finally, we provide a measure of the degree of tax progressiveness by the means of the coefficient of average tax progression:

$$
\Gamma\left(\tau^{F}, \tau^{H}, \kappa, p\right) \equiv \tau^{M}-\tau^{A}=\frac{\tau^{H} \kappa}{w^{F}},
$$

where $w^{F}=w\left(1+\tau^{F}\right)$ with $w=w\left(\tau^{F}, p\right)$.

As the average tax burden $\tau^{A}$ rises with wage rate, the system tax is progressive such that $\Gamma()>$.0 which holds as longer as $\kappa>0$. It is worth emphasizing that our approach which consists to define the average tax together with the marginal tax wedge by taking into account the wage paid by the firm allows for "scaling" the tax burden faced by households in terms of firms' labor cost, the index of average tax progression being expressed in terms of consumption goods; that's why we use the "wedge" label. By abstracting from this "scaling" approach, we would define the marginal and average tax wedges together with the coefficient of average tax progression as follows : $\tau^{M} \equiv \tau^{H} w, \tau^{A} \equiv \tau^{H}(w-\kappa)$ and $\Gamma \equiv \tau^{H} \kappa>0$ (as longer as $\kappa>0)$.

\section{Labor Tax Reform: A Fall in Payroll Taxes and a Rise in Labor Income Taxes}

In this section, we consider a labor tax strategy which involves simultaneously cutting a payroll tax by $\mathrm{d} \tau^{F}<0$ and increasing the labor income tax by $\mathrm{d} \tau^{H}>0$ so as to leave unchanged the marginal tax wedge, i. e. $\mathrm{d} \tau^{M}=0$. By making use of (140), the labor tax reform strategy requires a rise in the wage income tax by the following amount:

$$
\left.\mathrm{d} \tau^{H}\right|^{F, H} \equiv-\theta \mathrm{d} \tau^{F}, \quad \theta \equiv \frac{1-\tau^{H}}{1+\tau^{F}}<1 .
$$

From (143), the income wage tax must be increased by a smaller amount than the fall in $\operatorname{tau}^{F}$ for leaving unchanged the marginal tax wedge. Because we assumed initial positive payroll taxes so that the denominator of (140) is higher than unity, the fiscal reform keeping the 
marginal tax wedge constant does not yield to a change in $\tau^{H}$ by the same proportion than the fall in $\tau^{F}$.

Substituting the static solution for the wage rate (50) that holds in the long-run, and differentiating the coefficient of average tax progression (142) w. r. t. $\tau^{H}$ and $\tau^{F}$, and the using (143), we find that the fiscal reform raises the degree of average tax progression :

$$
\mathrm{d} \Gamma=-\frac{\kappa}{w^{F}} \theta \mathrm{d} \tau^{F}>0,
$$

where $\mathrm{d} \tau^{F}<0$ since we considered a fall in payroll taxes. The explanation comes from the fact that the wage rate is raised by the same proportion than the fall in $\tau^{F}$. Consequently, as longer as $\kappa>0$, the rise in $\tau^{H}$ leads to an increase in $\Gamma$.

Making use of long-term effects of permanent changes in $\tau^{F}$ and $\tau^{H}$ and substituting $\left.\mathrm{d} \tau^{H}\right|^{F, H}$ given by (143), we are able to estimate the directions and the sizes of the long-run changes of main economic variables after a fall in $\tau^{F}$ associated with a rise in $\tau^{H}$ by an amount that leaves unaffected $\tau^{M}$.

$$
\text { case } k^{N}>k^{T}
$$

If the non traded sector is relatively more capital intensive than the traded sector, the long-run changes are given by:

$$
\begin{aligned}
\left.\mathrm{d} \bar{\lambda}\right|^{F, H} & =\left.\lambda_{\tau^{H}} \mathrm{~d} \tau^{H}\right|^{F, H}+\lambda_{\tau^{F}} \mathrm{~d} \tau^{F} \\
& =\frac{\mathrm{d} \bar{\lambda}}{\mathrm{d} \tau^{F}} \frac{\kappa}{\tilde{\Lambda}} \frac{\left(1-\tau^{H}\right)}{\tilde{w}^{A}} \mathrm{~d} \tau^{F}=\frac{\mathrm{d} \bar{\lambda}}{\mathrm{d} \tau^{F}} \frac{\kappa}{\tilde{w}} \mathrm{~d} \tau^{F} \\
\left.\mathrm{~d} \tilde{x}\right|^{F, H} & =\left.\frac{\partial \tilde{x}}{\partial \tau^{H}} \mathrm{~d} \tau^{H}\right|^{F, H}+\frac{\partial \tilde{x}}{\partial \tau^{F}} \mathrm{~d} \tau^{F} \\
& =\frac{\partial \tilde{x}}{\partial \tau^{F}}\left[1-\theta \frac{(\tilde{w}-\kappa)\left(1+\tau^{F}\right)}{\tilde{w}\left(1-\tau^{H}\right)}\right] \mathrm{d} \tau^{F}=\frac{\mathrm{d} \tilde{x}}{\mathrm{~d} \tau^{F}} \frac{\kappa}{\tilde{w}} \mathrm{~d} \tau^{F},
\end{aligned}
$$

where $x=c, K, L, b$ and we used the fact that $\frac{\partial \tilde{x}}{\partial \tau^{H}}=\frac{(\tilde{w}-\kappa)\left(1+\tau^{F}\right)}{\tilde{w}\left(1-\tau^{H}\right)} \frac{\partial \tilde{x}}{\partial \tau^{F}}$. From (145b), the longrun changes of real consumption, physical capital and employment fiscal tax reform following a substitution of payroll taxes for income wage taxes are proportional to the steady-state variations after a change in $\tau^{F}$. While the directions of real expenditure in consumption goods and labor supply are ambiguous, we have been able to state that overall capital stock must rise after a fall in wage taxes paid by employers so that $\tilde{K}$ will be permanently increased after the fiscal tax reform. Interestingly, the larger tax allowances $\kappa$, the stronger the long-run stimulus of capital accumulation. This comes from the fact that a higher $\kappa$ softens the fall in the after-tax labor income and therefore the decrease in the marginal benefit of supplying labor and consequently moderates the that impinges negatively on $\tilde{L}$. In addition, as $\kappa$ gets larger, the combined effect of a smaller drop of employment and a lower reduction of the aftertax wage income $\tilde{w}^{A}$ moderates the wealth effect and therefore the decline in real expenditure in consumption goods.

It is now convenient to explore the overall effect of the fiscal tax reform on public revenue. First, while the fall in the tax rate paid by employers reduces the government revenues, they 
are raised by the increased income labor tax rate paid by households. Since the former is levied on a relatively larger tax basis than the former, i. e. $\tau^{H}$ increased by a smaller amount than the fall in $\tau^{F}$, for given wage rate and employment, labor fiscal revenues decreased. Second, the fiscal reform strategy makes households willing to spend a larger share of their available time to work and to raise their consumption expenditure, public revenues from labor taxes and VAT increase.

To estimate the overall effect of the fiscal reform strategy on public revenues, we differentiate the balanced government budget constraint (113):

$$
\mathrm{d} Z=\left.\tau^{c} p_{c} \mathrm{~d} \tilde{c}\right|^{F, H}+\left.\left(\tilde{w}^{F}-\tilde{w}^{A}\right) \mathrm{d} \tilde{L}\right|^{F, H}+\left\{\left[\tilde{w}+\left(\tau^{F}+\tau^{H}\right) w_{\tau^{F}}\right] \mathrm{d} \tau^{F}+\left.(\tilde{w}-\kappa) \mathrm{d} \tau^{H}\right|^{F, H}\right\} \tilde{L}
$$

where the steady-changes in real consumption and labor supply lead to the following variation in public revenues:

$$
\begin{aligned}
& \left.\tau^{c} p_{c} \mathrm{~d} \tilde{c}\right|^{F, H}+\left.\left(\tilde{w}^{F}-\tilde{w}^{A}\right) \mathrm{d} \tilde{L}\right|^{F, H} \\
= & -\frac{\sigma_{L} \tilde{L}}{\Delta} \kappa \tilde{\Lambda} \sigma_{c}\left\{p_{c} \tilde{c}\left[\left(1+\tau^{c}\right)-\frac{\tilde{w}^{A}}{\tilde{w}^{F}}\right]+\frac{r^{\star}}{\nu_{2}} \frac{\omega_{2}^{1}}{\nu_{2}}\left(\sigma_{c} \tilde{c}^{N}-\sigma_{L} \tilde{L} \tilde{k}^{T} \nu_{2}\right)\left(\tilde{c}^{N} \tau^{A}-\frac{p_{c} \tilde{c}}{\tilde{w}^{F}} \tau^{c} \tilde{k}^{T} \nu_{2}\right)\right\}(
\end{aligned}
$$

The sign of (147) follows from the fact that consumption and employment rise after a fall in the payroll tax $\tau^{F}$ so that government earnings grow. In addition, we computed the last term on the RHS of (113) which can be rewritten as follows:

$$
\left[\tilde{w}+\left(\tau^{F}+\tau^{H}\right) w_{\tau^{F}}\right] \tilde{L} \mathrm{~d} \tau^{F}+\left.(\tilde{w}-\kappa) \tilde{L} \mathrm{~d} \tau^{H}\right|^{F, H}=\kappa \theta \tilde{L} \tau^{F}<0 .
$$

case $k^{T}>k^{N}$

If the traded sector is relatively more capital intensive than the non traded sector, the long-run changes are given by:

$$
\begin{aligned}
\left.\mathrm{d} \bar{\lambda}\right|^{F, H} & =\left.\lambda_{\tau^{H}} \mathrm{~d} \tau^{H}\right|^{F, H}+\lambda_{\tau^{F}} \mathrm{~d} \tau^{F} \\
& =\frac{\mathrm{d} \bar{\lambda}}{\mathrm{d} \tau^{F}} \frac{\kappa}{\tilde{\Lambda}} \frac{\left(1-\tau^{H}\right)}{\tilde{w}^{A}} \mathrm{~d} \tau^{F}=\frac{\mathrm{d} \bar{\lambda}}{\mathrm{d} \tau^{F}} \frac{\kappa}{\tilde{w}} \mathrm{~d} \tau^{F}, \\
\left.\mathrm{~d} \tilde{x}\right|^{F, H} & =\left.\frac{\partial \tilde{x}}{\partial \tau^{H}} \mathrm{~d} \tau^{H}\right|^{F, H}+\frac{\partial \tilde{x}}{\partial \tau^{F}} \mathrm{~d} \tau^{F} \\
& =\frac{\partial \tilde{x}}{\partial \tau^{F}}\left[1-\theta \frac{(\tilde{w}-\kappa)\left(1+\tau^{F}\right)}{\tilde{w}\left(1-\tau^{H}\right)}\right] \mathrm{d} \tau^{F}=\frac{\mathrm{d} \tilde{x}}{\mathrm{~d} \tau^{F}} \frac{\kappa}{\tilde{w}} \mathrm{~d} \tau^{F},
\end{aligned}
$$

where $x=c, K, L, b$ and we used the fact that $\frac{\partial \tilde{x}}{\partial \tau^{H}}=\frac{(\tilde{w}-\kappa)\left(1+\tau^{F}\right)}{\tilde{w}\left(1-\tau^{H}\right)} \frac{\partial \tilde{x}}{\partial \tau^{F}}$. From (149b), the long-run changes of real consumption, physical capital and employment fiscal tax reform following a substitution of payroll taxes for income wage taxes are proportional to the steadystate variations after a change in $\tau^{F}$. Consequently, according to (100), the fiscal tax reform unambiguously raises real consumption and labor supply, and stimulates capital accumulation in the long-run. 
By substituting of long-run changes of real consumption and employment, we obtain:

$$
\begin{aligned}
\mathrm{d} Z & =\left\{-\frac{\sigma_{c} p_{c} \tilde{c} \sigma_{L} \tilde{L} \tilde{\Lambda}}{\Delta}\left(\tau^{c}+\tau^{A}\right)+\kappa\left(\frac{1-\tau^{H}}{1+\tau^{F}}\right) \tilde{L}\right\} \mathrm{d} \tau^{F} \\
& =\frac{\sigma_{L} \tilde{w}\left(1-\tau^{H}\right) \tilde{L}}{\Delta} \tilde{w} \tilde{L}\left\{-\frac{\sigma_{c} p_{c} \tilde{c}}{\tilde{w}^{A} \tilde{L}}\left(\tau^{c}+\tau^{A}\right)+\frac{\kappa}{\tilde{w}}\left(\frac{\sigma_{c} p_{c} \tilde{c}}{\sigma_{L} \tilde{w}^{F} \tilde{L}}+1\right)\right\} \mathrm{d} \tau^{F},
\end{aligned}
$$

The change in government revenues following the fiscal reform strategy is unclear. While the substitution of labor income taxes for payroll taxes improve the earnings of the state by raising real consumption (hence the fiscal basis of consumption taxes) together with labor (hence the fiscal basis of labor taxes), the fall in government revenues due to the insufficient rise in the labor income tax rate plays in opposite direction. The latter effect must predominate over the former effect so that government revenues fall. The explanation is as follows. Since the labor income tax rate must rise by an amount $\frac{\tilde{w}}{\tilde{w}-\kappa} \frac{1-\tau^{H}}{1+\tau^{F}}$ to maintain the government budget constraint balanced, the increase in $\tau^{H}$ is not large enough for this condition to be fulfilled, since the labor income tax rate rises by $\frac{1-\tau^{H}}{1+\tau^{F}}$ which is smaller.

\section{J Tax Multipliers}

In this section, we derive analytical expressions of overall and sectoral tax multipliers.

\section{J.1 Overall Tax Multipliers}

\section{Long-Run Tax Multiplier}

Because overall output denoted by $Y$ is the sum of traded output $Y^{T}$ and non traded output measured in terms of the traded good $\frac{p}{\mu} Y^{N}$, using the fact that $Y^{T} \equiv Y^{T}(K, L, p)$ and $Y^{N} \equiv Y^{N}(K, L, p)$, remembering that steady-state level of the real exchange rate is unaffected by a tax restructuring, the steady-state change of overall output can be expressed as:

$$
\begin{aligned}
\left.\mathrm{d} \tilde{Y}\right|^{j, k} & =\left.\left(Y_{K}^{T}+\frac{\tilde{p}}{\mu} Y_{K}^{N}\right) \mathrm{d} \tilde{K}\right|^{j, k}+\left.\left(Y_{L}^{T}+\frac{\tilde{p}}{\mu} Y_{L}^{N}\right) \mathrm{d} \tilde{L}\right|^{j, k} \\
& =\left.\tilde{p} r^{\star} \mathrm{d} \tilde{K}\right|^{j, k}+\left.w^{F} \mathrm{~d} \tilde{L}\right|^{j, k}>0 .
\end{aligned}
$$

where we use properties (57b) and (57c) to get (151).

\section{Initial Tax Multiplier}

Adopting a similar procedure keeping in mind that the capital stock is initially predetermined, the short-run tax multiplier writes as follows:

$$
\begin{aligned}
\left.\mathrm{d} Y(0)\right|^{j, k} & =\left.\left(Y_{L}^{T}+\frac{p}{\mu} Y_{L}^{N}\right) \mathrm{d} L(0)\right|^{j, k}+\left.\left(\hat{Y}_{p}^{T}+\frac{p}{\mu} \hat{Y}_{p}^{N}\right) \mathrm{d} p(0)\right|^{j, k}, \\
& =\left.w^{F} \mathrm{~d} L(0)\right|^{j, k}>0,
\end{aligned}
$$

where we use properties (57c) to get (152); according to property (57a), denoting by a hat the partial derivative of $Y$ w. r. t. $p$ for given labor, $\hat{Y}_{p}^{T}+\frac{p}{\mu} \hat{Y}_{p}^{N}=0$; 


\section{J.2 Sectoral Tax Multipliers}

\section{Long-Run Sectoral Tax Multipliers}

$k^{N}>k^{T}$

We calculate the tax multiplier in the traded sector by differentiating the short-run static solution for $Y^{T}$ evaluated at the steady-state:

$$
\begin{aligned}
\left.\mathrm{d} \tilde{Y}^{T}\right|^{j, k} & =\left.Y_{K}^{T} \mathrm{~d} \tilde{K}\right|^{j, k}+\left.Y_{L}^{T} \mathrm{~d} \tilde{L}\right|^{j, k}=\Phi^{j, k}\left[Y_{K}^{T} \frac{\mathrm{d} \tilde{K}}{\mathrm{~d} \tau^{j}}+Y_{L}^{T} \frac{\mathrm{d} \tilde{L}}{\mathrm{~d} \tau^{j}}\right] \mathrm{d} \tau^{F}, \\
& =\frac{\nu_{1}}{\nu_{2}} \frac{\sigma_{L} \tilde{L}}{\Delta} \sigma_{c} p_{c} \tilde{c} \Upsilon^{j}\left\{\left[\left(1-\alpha_{c}\right) \tilde{w}^{F}+r^{\star} \tilde{p} \tilde{k}^{N}\right]\right. \\
& \left.+\alpha_{c} \tilde{k}^{N} r^{\star} \frac{\omega_{2}^{1}}{\nu_{2}}\left(\sigma_{c} \tilde{c}^{N}-\sigma_{L} \tilde{L} k^{T} \nu_{2} \tilde{\Lambda}\right)\right\} \Phi \mathrm{d} \tau^{j}>0, \quad j=F, H,
\end{aligned}
$$

where $\mathrm{d} \tau^{j}<0$ since we consider a fall in the labor income tax rate $\tau^{j}, \Phi^{j, c}=$ $\frac{p_{c} \tilde{c}}{\chi_{c}}\left[1-\frac{\tilde{w}^{A} \tilde{L}}{p_{c}\left(1+\tau^{c}\right) \tilde{c}}\right]>0($ with $j=F, H)$ as longer as $\tilde{a}>0$, and $\Phi^{F, H}=\frac{\tilde{w}}{\kappa}>0$. To determine (153), we used the fact that $Y_{L}^{T}=-\tilde{p} \nu_{1} \tilde{k}^{N}>0, Y_{K}^{T}=\tilde{p} \nu_{1}<0,\left(\nu_{2} \tilde{k}^{T}+\nu_{1} \tilde{k}^{N}\right)=-\frac{\tilde{w}^{F}}{\tilde{p}}<0$ and $\nu_{1}+\nu_{2}=r^{\star}$.

We calculate the tax multiplier in the non traded sector by differentiating the short-run static solution for $Y^{N} / \mu$ evaluated at the steady-state:

$$
\begin{aligned}
\left.\frac{\tilde{p}}{\mu} \mathrm{d} \tilde{Y}^{N}\right|^{j, k} & =\left.\frac{\tilde{p}}{\mu} Y_{K}^{N} \mathrm{~d} \tilde{K}\right|^{j, k}+\left.\frac{\tilde{p}}{\mu} Y_{L}^{N} \mathrm{~d} \tilde{L}\right|^{j, k}=\Phi^{j, k}\left[Y_{K}^{N} \frac{\mathrm{d} \tilde{K}}{\mathrm{~d} \tau^{j}}+\frac{Y_{L}^{N}}{\mu} \frac{\mathrm{d} \tilde{L}}{\mathrm{~d} \tau^{j}}\right] \mathrm{d} \tau^{j} \\
& =-\frac{\sigma_{L} \tilde{L}}{\Delta} \sigma_{c} \tilde{c}^{N} \Upsilon^{j}\left\{\tilde{w}^{F}-\tilde{k}^{T} r^{\star} \frac{\omega_{2}^{1}}{\nu_{2}}\left(\sigma_{c} \tilde{c}^{N}-\sigma_{L} \tilde{L} k^{T} \nu_{2} \tilde{\Lambda}\right)\right\} \Phi \mathrm{d} \tau^{j}>0
\end{aligned}
$$

where we used the fact that $Y_{K}^{N}=\mu \nu_{2}>0$ and $Y_{L}^{N}=-\tilde{k}^{T} \mu \nu_{2}<0$.

$$
k^{T}>k^{N}
$$

We calculate the tax multiplier in the traded sector by differentiating the short-run static solution for $Y^{T}$ evaluated at the steady-state:

$$
\begin{aligned}
\left.\mathrm{d} \tilde{Y}^{T}\right|^{j, k} & =\left.Y_{K}^{T} \mathrm{~d} \tilde{K}\right|^{j, c}+\left.Y_{L}^{T} \mathrm{~d} \tilde{L}\right|^{j, c}=\Phi^{j, k}\left[Y_{K}^{T} \frac{\mathrm{d} \tilde{K}}{\mathrm{~d} \tau^{j}}+Y_{L}^{T} \frac{\mathrm{d} \tilde{L}}{\mathrm{~d} \tau^{j}}\right] \mathrm{d} \tau^{F}, \\
& =\frac{\nu_{2}}{\nu_{1}} \frac{\sigma_{L} \tilde{L}}{\Delta} \Upsilon^{j} \sigma_{c} p_{c} \tilde{c}\left[\left(1-\alpha_{c}\right) \tilde{w}^{F}+r^{\star} \tilde{p} \tilde{k}^{N}\right] \Phi^{j, k} \mathrm{~d} \tau^{j}>0, \quad j=F, H,
\end{aligned}
$$

where $\Upsilon^{j}>0$ and $\Phi^{j, k}>0$ (as longer as $\tilde{a}>0$. We used the fact that $Y_{L}^{T}=-\tilde{p} \nu_{2} \tilde{k}^{N}<0$, $Y_{K}^{T}=\tilde{p} \nu_{2}>0,\left(\nu_{1} \tilde{k}^{T}+\nu_{2} \tilde{k}^{N}\right)=-\frac{\tilde{w}^{F}}{\tilde{p}}<0$ and $\nu_{1}+\nu_{2}=r^{\star}$ to get $(155)$.

We calculate the tax multiplier in the non traded sector by differentiating the short-run static solution for $Y^{N} / \mu$ evaluated at the steady-state:

$$
\begin{aligned}
\left.\frac{\tilde{p}}{\mu} \mathrm{d} \tilde{Y}^{N}\right|^{j, k} & =\left.\frac{\tilde{p}}{\mu} Y_{K}^{N} \mathrm{~d} \tilde{K}\right|^{j, k}+\left.\frac{\tilde{p}}{\mu} Y_{L}^{N} \mathrm{~d} \tilde{L}\right|^{j, k}=\Phi^{j, k}\left[\frac{Y_{K}^{N}}{\mu} \frac{\mathrm{d} \tilde{K}}{\mathrm{~d} \tau^{j}}+\frac{Y_{L}^{N}}{\mu} \frac{\mathrm{d} \tilde{L}}{\mathrm{~d} \tau^{j}}\right] \mathrm{d} \tau^{j}, \\
& =-\frac{\sigma_{L} \tilde{L}}{\Delta} \Upsilon^{j} \sigma_{c} \tilde{c}^{N} \tilde{w}^{F} \Phi^{j, k} \mathrm{~d} \tau^{j}>0, \quad j=F, H
\end{aligned}
$$


where we used the fact that $Y_{K}^{N}=\mu \nu_{1}<0$ and $Y_{L}^{N}=-\tilde{k}^{T} \mu \nu_{1}>0$ to get (156).

\section{Short-Run Sectoral Tax Multipliers}

$$
k^{N}>k^{T}
$$

Remembering that the short-run solution $Y^{T} \equiv Y^{T}(K, L, p)$, using the fact that the capital stock is initially predetermined, $\left.\mathrm{d} L(0)\right|^{j, k}=\left.\mathrm{d} \tilde{L}\right|^{j, k}+\left.L_{p} \mathrm{~d} p(0)\right|^{j, k}$ and $\left.\mathrm{d} p(0)\right|^{j, k}=-\left.\omega_{2}^{1} \mathrm{~d} \tilde{K}\right|^{j, k}$, the short-run tax multiplier is given by:

$$
\begin{aligned}
\left.\mathrm{d} Y^{T}(0)\right|^{j, k} & =\left.Y_{L}^{T} \mathrm{~d} L(0)\right|^{j, k}+\left.\hat{Y}_{p}^{T} \mathrm{~d} p(0)\right|^{j, k}, \\
& =-\Phi^{j, k}\left[\tilde{p} \nu_{1} \tilde{k}^{N} \frac{\mathrm{d} \tilde{L}}{\mathrm{~d} \tau^{j}}+Y_{p}^{T} \omega_{2}^{1} \frac{\mathrm{d} \tilde{K}}{\mathrm{~d} \tau^{j}}\right] \mathrm{d} \tau^{j} \gtrless 0,
\end{aligned}
$$

where we used the fact that $Y_{L}^{T}=-\tilde{p} \nu_{1} \tilde{k}^{N}>0$; we denoted by a hat the partial derivative of $Y^{T}$ w. r. t. $p$ for given labor, i. e. $\hat{Y}_{p}^{T}<0$, and we used the fact that $Y_{L}^{T} L_{p}+\hat{Y}_{p}^{T}=Y_{p}^{T}$. The short-run tax multiplier in the traded sector is the result of two conflictory forces: while the initial stimulus of labor supply induces a labor inflow in the traded sector, the real exchange appreciation shifts away resources from the traded sector towards the non traded sector.

Differentiating the short-run solution for $Y^{N} \equiv Y^{N}(K, L, p)$ and remembering that the capital stock is initially predetermined, the short-run tax multiplier is given by:

$$
\begin{aligned}
\left.\frac{p}{\mu} \mathrm{d} Y^{N}(0)\right|^{j, k} & =\left.\frac{p}{\mu} Y_{L}^{N} \mathrm{~d} L(0)\right|^{j, k}+\left.\frac{p}{\mu} \hat{Y}_{p}^{N} \mathrm{~d} p(0)\right|^{j, k}, \\
& =-\Phi^{j, k}\left[\tilde{p} \nu_{1} \tilde{k}^{N} \frac{\mathrm{d} \tilde{L}}{\mathrm{~d} \tau^{j}}+\frac{Y_{p}^{N}}{\mu} \omega_{2}^{1} \frac{\mathrm{d} \tilde{K}}{\mathrm{~d} \tau^{j}}\right] \mathrm{d} \tau^{j} \gtrless 0,
\end{aligned}
$$

where we used the fact that $Y_{L}^{N}=-\tilde{k}^{T} \mu \nu_{2}<0$, and we denoted by a hat the partial derivative of $Y^{N}$ w. r. t. $p$ for given labor, i. e. $\hat{Y}_{p}^{N}>0$. The short-run tax multiplier in the non traded sector is the result of two conflictory forces: while the initial stimulus of labor supply induces a labor outflow from the non traded sector, the real exchange appreciation attracts resources in the non traded sector.

$$
k^{T}>k^{N}
$$

Remembering that the short-run solution $Y^{T} \equiv Y^{T}(K, L, p)$, using the fact that the capital stock is initially predetermined and the real exchange is unaffected by a tax restructuring, the short-run tax multiplier is given by:

$$
\left.\mathrm{d} Y^{T}(0)\right|^{j, k}=\left.Y_{L}^{T} \mathrm{~d} L(0)\right|^{j, k}=\left.Y_{L}^{T} \mathrm{~d} \tilde{L}\right|^{j, k}<0,
$$

where we used the fact that $Y_{L}^{T}=-\tilde{p} \nu_{2} \tilde{k}^{N}<0$,

Differentiating the short-run solution for $Y^{N} \equiv Y^{N}(K, L, p)$ and remembering that the capital stock is initially predetermined, the short-run tax multiplier is given by:

$$
\left.\frac{p}{\mu} \mathrm{d} Y^{N}(0)\right|^{j, k}=\left.\frac{p}{\mu} Y_{L}^{N} \mathrm{~d} L(0)\right|^{j, k}=\left.\frac{p}{\mu} Y_{L}^{N} \mathrm{~d} \tilde{L}\right|^{j, k}>0,
$$

where we used the fact that $Y_{L}^{N}=-\tilde{k}^{T} \mu \nu_{1}>0$. 


\section{K Welfare Analysis}

In this section, we investigate the welfare effects of an unanticipated of tax restructuring which involves simultaneously cutting labor tax by $\mathrm{d} \tau^{j}<0(j=F, H)$ and raising the consumption tax rate or the labor income tax rate by $\mathrm{d} \tau^{k}>0(k=c, H)$. We denote by $\phi$ the instantaneous welfare:

$$
\phi(t)=u(c(t))+v(L(t)),
$$

and by $U$ its discounted value over an infinite horizon:

$$
U=\int_{0}^{\infty} \phi(t) \exp (-\delta t) \mathrm{d} t
$$

\section{K.1 Instantaneous Welfare}

We first linearize the instantaneous utility function (161) in the neighborhood of the steadystate:

$$
\phi(t)=\tilde{\phi}+u_{c}(\tilde{c})(c(t)-\tilde{c})+v_{L}(\tilde{L})(L(t)-\tilde{L})
$$

with $\tilde{\phi}$ given by

$$
\tilde{\phi}=u(\tilde{c})+v(\tilde{L})
$$

By substituting solutions for $c(t)$ and $L(t)$, we obtain the stable solution for instantaneous welfare:

$$
\phi(t)=\tilde{\phi}+\left[u_{c} c_{p}+v_{L} L_{p}\right] \omega_{2}^{1}\left(K_{0}-\tilde{K}\right) e^{\nu_{1} t},
$$

where partial derivatives are evaluated at the steady-state, i. e. $u_{c}=u_{c}(\tilde{c})$ and $v_{L}=v_{L}(\tilde{L})$. We estimate the expression in square brackets by making use of the first-order conditions for consumption and labor supply supply decisions evaluated at the steady-state, i. e. $u_{c}=$ $p_{c} \bar{\lambda}\left(1+\tau^{c}\right)$ and $v_{L}=-\bar{\lambda} \tilde{w}^{A}$. We obtain:

$$
u_{c} c_{p}+v_{L} L_{p}=\bar{\lambda}\left[-\sigma_{c} \tilde{c}^{N}\left(1+\tau^{c}\right)+\frac{\tilde{w}^{A}}{\tilde{w}^{F}} \sigma_{L} \tilde{L} \tilde{k}^{T} \tilde{\Lambda} \frac{\tilde{h}}{\mu\left(\tilde{k}^{N}-\tilde{k}^{T}\right)}\right] \gtrless 0 .
$$

Evaluate (161) at the steady-state and differentiate, one obtains the long-run change of $\phi$ after a tax restructuring:

$$
\left.\mathrm{d} \tilde{\phi}\right|^{j, k}=\Phi^{j, k}\left[u_{c} \frac{\mathrm{d} \tilde{c}}{\mathrm{~d} \tau^{j}} \mathrm{~d} \tau^{j}+v_{L} \frac{\mathrm{d} \tilde{L}}{\mathrm{~d} \tau^{j}}\right] \mathrm{d} \tau^{j} \gtrless 0,
$$

where $0<\Phi^{j, k}<1$.

Evaluate (165) at time $t=0$ and differentiate, we get the initial reaction of $\phi$ after a tax restructuring:

$$
\begin{aligned}
\left.\mathrm{d} \phi(0)\right|^{j, k} & =\left.\mathrm{d} \tilde{\phi}\right|^{j, k}-\left.\left(u_{c} c_{p}+v_{L} L_{p}\right) \omega_{2}^{1} \mathrm{~d} \tilde{K}\right|^{j, k} \\
& =\Phi^{j, k}\left\{u_{c}\left[\frac{\mathrm{d} \tilde{c}}{\mathrm{~d} \tau^{j}}-c_{p} \omega_{2}^{1} \frac{\mathrm{d} \tilde{K}}{\mathrm{~d} \tau^{j}}\right]+v_{L}\left[\frac{\mathrm{d} \tilde{L}}{\mathrm{~d} \tau^{j}}-L_{p} \omega_{2}^{1} \frac{\mathrm{d} \tilde{K}}{\mathrm{~d} \tau^{j}}\right]\right\} \mathrm{d} \tau^{j},
\end{aligned}
$$


where we used (167) to get (168).

Case $k^{N}>k^{T}$

If the non traded sector is more capital intensive than the traded sector, the long-run change of $\phi$ after a tax restructuring writes as follows:

$$
\begin{aligned}
\left.\mathrm{d} \tilde{\phi}\right|^{j, k} & =\Phi^{j, k}\left\{p_{c} \bar{\lambda}\left(1+\tau^{c}\right)\left[1-\frac{\tilde{w}^{A}}{\tilde{w}^{F}} \frac{1}{p_{c}\left(1+\tau^{c}\right)}\right] \frac{\mathrm{d} \tilde{c}}{\mathrm{~d} \tau^{j}} \mathrm{~d} \tau^{j}\right. \\
& \left.+\Gamma^{j} \bar{\lambda} \frac{\sigma_{L} \tilde{L}}{\Delta} \tilde{w}^{A} \frac{\sigma_{c} p_{c} \tilde{c}}{\tilde{p}} \frac{r^{\star}}{\nu_{2}} \frac{\omega_{2}^{1}}{\nu_{2}}\left(\sigma_{c} \tilde{c}^{N}-\sigma_{L} \tilde{L} \tilde{k}^{T} \nu_{2} \tilde{\Lambda}\right)\left(\alpha_{c}+\frac{\tilde{k}^{T} \tilde{p} \nu_{2}}{\tilde{w}^{F}}\right)\right\} \mathrm{d} \tau^{j} \gtrless 0,
\end{aligned}
$$

where $0<\Phi^{j, k}<1$ and $\Gamma^{j}>0$. We substituted $u_{c}=p_{c} \bar{\lambda}\left(1+\tau^{c}\right)$ and $v_{L}=-\bar{\lambda} \tilde{w}^{A}$, and we used the fact that $\frac{\mathrm{d} \tilde{L}}{\mathrm{~d} \tau^{j}}=\frac{p_{c}}{\tilde{w}^{F}} \frac{\mathrm{d} \tilde{c}}{\mathrm{~d} \tau^{j}}-\Gamma^{j} \frac{\sigma_{L} \tilde{L}}{\Delta} \frac{r^{\star}}{\nu_{2}} \frac{\omega_{2}^{1}}{\nu_{2}}\left(\sigma_{c} \tilde{c}^{N}-\sigma_{L} \tilde{L} \tilde{k}^{T} \nu_{2} \tilde{\Lambda}\right) \sigma_{c} \tilde{c}^{N}$ to determine (169). Since the sign of the long-run change of instantaneous welfare is not clear-cut, we dot not calculate the initial reaction of $\phi$ which in particular depends on expression (169).

Case $k^{T}>k^{N}$

If the traded sector is more capital intensive than the non traded sector, the long-run change of $\phi$ after a tax restructuring writes as follows:

$$
\left.\mathrm{d} \tilde{\phi}\right|^{j, k}=\Phi^{j, k} p_{c} \bar{\lambda}\left(1+\tau^{c}\right)\left[1-\frac{\tilde{w}^{A}}{\tilde{w}^{F}} \frac{1}{1+\tau^{c}}\right] \frac{\mathrm{d} \tilde{c}}{\mathrm{~d} \tau^{j}} \mathrm{~d} \tau^{j}>0,
$$

where $0<\Phi^{j, k}<1$. We substituted $u_{c}=p_{c} \bar{\lambda}\left(1+\tau^{c}\right)$ and $v_{L}=-\bar{\lambda} \tilde{w}^{A}$, and we used the fact that $\frac{\mathrm{d} \tilde{L}}{\mathrm{~d} \tau^{j}}=\frac{p_{c}}{\tilde{w}^{F}} \frac{\mathrm{d} \tilde{c}}{\mathrm{~d} \tau^{j}}$ to determine (170).

\section{K.2 Overall Welfare}

Until now, we have analyzed the instantaneous welfare implications of an unanticipated permanent fiscal expansion, say at different points of times. To address welfare effects in a convenient way within an intertemporal-maximizing framework, we have to evaluate the discounted value of (161) over the agent's infinite planning horizon. Whereas the change of overall welfare will be estimated numerically, we determine its measure along a transitional path after a tax restructuring.

In order to have a correct and comprehensive measure of welfare, we calculate first the discounted value of instantaneous welfare over the entire planning horizon:

$$
\begin{aligned}
U & =\frac{\tilde{\phi}}{\delta}+\frac{\left[u_{c} c_{p}+v_{L} L_{p}\right] \omega_{2}^{1}}{r^{\star}-\nu_{1}} A_{1} \\
& =\frac{\tilde{\phi}}{\delta}+\frac{\phi(0)-\tilde{\phi}}{r^{\star}-\nu_{1}} .
\end{aligned}
$$

The first term on the right hand-side of (171) represents the capitalized value of instantaneous welfare evaluated at the steady-state. The second term on the RHS of (171) vanishes whenever the traded sector is more capital intensive than the non traded sector since the dynamics of the real exchange degenerate. If consumption reacts strongly on impact and labor is not too 
much responsive, then $\phi(0)$ can overshoot its long-run level which exerts a positive influence on overall welfare.

\section{Case $k^{N}>k^{T}$}

If the non traded sector is more capital intensive than the traded sector, the long-run change of $\phi$ after a tax restructuring writes as follows:

$$
\begin{aligned}
\left.\mathrm{d} U\right|^{j, k} & =\left.\frac{1}{\delta} \mathrm{d} \tilde{\phi}\right|^{j, k}-\left.\frac{\left[u_{c} c_{p}+v_{L} L_{p}\right] \omega_{2}^{1}}{r^{\star}-\nu_{1}} \mathrm{~d} \tilde{K}\right|^{j, k} \\
& =\frac{1}{r^{\star} \nu_{2}} \Phi^{j, k}\left\{u_{c}\left[\frac{\mathrm{d} \tilde{c}}{\mathrm{~d} \tau^{j}}-r^{\star} c_{p} \omega_{2}^{1} \frac{\mathrm{d} \tilde{K}}{\mathrm{~d} \tau^{j}}\right]+v_{L}\left[\frac{\mathrm{d} \tilde{L}}{\mathrm{~d} \tau^{j}}-r^{\star} L_{p} \omega_{2}^{1} \frac{\mathrm{d} \tilde{K}}{\mathrm{~d} \tau^{j}}\right]\right\} \mathrm{d} \tau^{j} \gtrless 0,(172)
\end{aligned}
$$

with

$$
\begin{aligned}
\frac{\mathrm{d} \tilde{c}}{\mathrm{~d} \tau^{j}}-r^{\star} c_{p} \omega_{2}^{1} \frac{\mathrm{d} \tilde{K}}{\mathrm{~d} \tau^{j}} & =\frac{\sigma_{L} \tilde{L}}{\Delta} \sigma_{c} \tilde{c} \Upsilon^{j}\left\{\tilde{p} \nu_{1} \tilde{k}^{N}\left[1+\alpha_{c} \sigma_{c} \frac{\tilde{c}^{N}}{\tilde{p}} \frac{r^{\star}}{\nu_{2}} \omega_{2}^{1}\right]\right. \\
& \left.+r^{\star} \tilde{k}^{T} \nu_{2} \omega_{2}^{1}\left[\sigma_{c} \tilde{c}^{N} \alpha_{c}-\sigma_{L} \tilde{L} \tilde{k}^{T} \tilde{\Lambda}\right]\right\}<0, \\
\frac{\mathrm{d} \tilde{L}}{\mathrm{~d} \tau^{j}}-r^{\star} L_{p} \omega_{2}^{1} \frac{\mathrm{d} \tilde{K}}{\mathrm{~d} \tau^{j}} & =-\frac{\sigma_{L} \tilde{L}}{\Delta} \sigma_{c} \tilde{c} \Upsilon^{j}\left\{\nu_{2}\left[1+\alpha_{c} \sigma_{c} \frac{\tilde{c}^{N}}{\tilde{p}} \frac{r^{\star}}{\nu_{2}} \frac{\omega_{2}^{1}}{\nu_{2}}\right]\right. \\
& \left.+\frac{r^{\star}}{\nu_{2}} \frac{\omega_{2}^{1}}{\tilde{p}} \sigma_{L} \tilde{L} \tilde{k}^{T} \tilde{\Lambda}\left[\alpha_{c}\left(1-\sigma_{c}\right)+\frac{\tilde{p} \nu_{2} \tilde{k}^{T}}{\tilde{w}^{F}}\right]\right\} \lessgtr 0 .
\end{aligned}
$$

Case $k^{T}>k^{N}$

If the traded sector is more capital intensive than the non traded sector, the long-run change of $\phi$ after a tax restructuring writes as follows:

$$
\left.\mathrm{d} U\right|^{j, k}=\left.\frac{1}{\delta} \mathrm{d} \tilde{\phi}\right|^{j, k}>0,
$$

where $\left.\mathrm{d} \tilde{\phi}\right|^{j, k}>0$ is given by (170).

\section{Imperfect Competition: Derivation of Price Index and Ag- gregate Non Traded Consumption}

The open economy consists of perfectly competitive firms producing a traded good and imperfectly competitive firms that each produces a single variety of a diversified good. These goods are similar but imperfect substitutes in consumption. We assume that there is a continuum of commodities indexed by the subscript $j \in[0, n]$ and denoted by $x(j)$, each produced by a different firm. Since commodities are differentiated, each firm has market power and is able to set a price over the marginal cost. Consumption of non traded commodity $j$ is aggregated by the means of the following formulation:

$$
c^{N}=n^{\frac{1}{1-\sigma}}\left[\int_{0}^{n}(x(j))^{\frac{\sigma-1}{\sigma}}\right]^{\frac{\sigma}{\sigma-1}}, \quad \sigma>1,
$$


where the size of the parameter $\sigma$ reflects the ease with which any varieties can be substituted for each other. The maximization problem writes as follows:

$$
\max _{x(j)} c^{N}=n^{\frac{1}{1-\sigma}}\left[\int_{0}^{n}(x(j))^{\frac{\sigma-1}{\sigma}}\right]^{\frac{\sigma}{\sigma-1}} \quad \text { subject to } \quad E^{N}=\int_{0}^{n} \varrho(j) x(j) \mathrm{d} j,
$$

where $E^{N}$ is any fixed nominal expenditure on non tradables. The first order conditions of problem are the constraint of problem (176) together with

$$
\left(\frac{x(j)}{x\left(j^{\prime}\right)}\right)^{-\frac{1}{\sigma}}=\frac{\varrho(j)}{\varrho\left(j^{\prime}\right)} \quad \text { or } \quad x(j)=\left(\frac{\varrho(j)}{\varrho\left(j^{\prime}\right)}\right)^{-\sigma} x\left(j^{\prime}\right),
$$

where we consider two goods, $j$ and $j^{\prime}$.

Consider now the aggregate price index $p$ corresponding to the real consumption index (175) defined as the minimum expenditure required to purchase one unit of real aggregate consumption $c^{N}=1$ :

$$
p=\min _{x(j)} E^{N}=\min _{x(j)} \int_{0}^{n} \varrho(j) x(j) \mathrm{d} j \quad \text { subject to } \quad c^{N}=n^{\frac{1}{1-\sigma}}\left[\int_{0}^{n}(x(j))^{\frac{\sigma-1}{\sigma}}\right]^{\frac{\sigma}{\sigma-1}}=1 .
$$

Substitution of (177) into (175) and expression of $E^{N}$, by keeping in mind that $c^{N}=$ $n^{\frac{1}{1-\sigma}}\left[\int_{0}^{n}(x(j))^{\frac{\sigma-1}{\sigma}}\right]^{\frac{\sigma}{\sigma-1}}=1$ implies that $n^{-\frac{1}{\sigma}} \int_{0}^{n}(x(j))^{\frac{\sigma-1}{\sigma}}=1$ leads to:

$$
\begin{aligned}
E^{N} & =\frac{x\left(j^{\prime}\right)}{\varrho\left(j^{\prime}\right)^{-\sigma}} \int_{0}^{n} \varrho(j)^{1-\sigma} \mathrm{d} j \\
1 & =n^{-\frac{1}{\sigma}} \frac{c\left(j^{\prime}\right)^{\frac{\sigma-1}{\sigma}}}{p\left(j^{\prime}\right)^{1-\sigma}} \int_{0}^{n} \varrho(j)^{1-\sigma} \mathrm{d} j,
\end{aligned}
$$

Dividing the first expression by the second gives:

$$
E^{N}=\left[\frac{n x\left(j^{\prime}\right)}{\varrho\left(j^{\prime}\right)^{-\sigma}}\right]^{\frac{1}{\sigma}}
$$

Substituting (180) into equation (179a), we obtain:

$$
\left(E^{N}\right)^{1-\sigma}=n^{-1} \int_{0}^{n} \varrho(j)^{1-\sigma} \mathrm{d} j
$$

By the definition of the price index, the unit cost of the non traded composite good writes as follows:

$$
p=n^{-\frac{1}{1-\sigma}}\left[\int_{0}^{n}(p(j))^{1-\sigma}\right]^{\frac{1}{1-\sigma}} .
$$

Using (179a) and (182), we obtain:

$$
\frac{x(j)^{\prime}}{\varrho\left(j^{\prime}\right)^{-\sigma}}=E^{N}(p)^{\sigma-1} n^{-1} .
$$

Substituting (183) into the second equality of (177), we obtain the demand for variety $j$ of the non traded consumption good:

$$
x(j)=\frac{1}{n}\left(\frac{\varrho(j)}{p}\right)^{-\sigma} c^{N},
$$

where we used the fact that $E^{N} / p=c^{N}$ with $c^{N}$ the composite non traded consumption good. 


\section{Data and Estimation Methodology}

The calibration of output shares of capital income $\left(\theta^{T}, \theta^{N}\right)$ and markups $\left(\mu^{T}, \mu^{N}\right)$ are based on the EU KLEMS sectoral database, comprising 11 industries and 13 countries over the period 1970-2004. ${ }^{3}$ Following De Gregorio, Giovannini and Wolf [1994], Agriculture, Hunting, Forestry and Fishing; Mining and Quarrying; Total Manufacturing; Transport and Storage; and Communication are classified as traded goods with weights given by relative nominal value added within the sector. Electricity, Gas and Water Supply; Construction; Wholesale and Retail Trade; Hotels and Restaurants; Finance, Insurance, Real Estate and Business Services; and Community Social and Personal Services industries account for the non traded sector. The dataset enables construction of aggregate data on traded and non traded output, capital stock and employment. Sectoral ratios $Y^{N} / Y$ and $L^{N} / L$ immediately follow.

Markups are estimated at the industry level for each country and are aggregated as follows to construct $\mu^{T}$ and $\mu^{N}$ :

$$
\mu^{T}=\sum_{j=1}^{4} \omega_{j T} \hat{\mu}_{j} \quad \mu^{N}=\sum_{j=1}^{6} \omega_{j N} \hat{\mu}_{j},
$$

where $\omega_{j T}\left(\right.$ resp. $\left.\omega_{j N}\right)$ is the nominal value added-weight of industry $j$ in sector $T$ (resp. $N)$. The estimates $\hat{\mu}_{j}$ are obtained applying the consistent methodology developed by Roeger [1995]. This approach takes account of labor, capital and intermediate inputs as production factors and the specific variables required to apply the Roeger's method are the following: gross output (at basic current prices), compensation of employees, intermediate inputs at current purchasers prices, and capital services (volume) indices. The testable equation of the Roeger's methodology may be written as:

$$
y_{j, t}=\beta_{j} x_{j, t}+\varepsilon_{j, t},
$$

with $y_{t}=\Delta\left(p_{j, t} Y_{j, t}\right)-\alpha_{N, t} \Delta\left(w_{j, t} L_{j, t}\right)-\alpha_{M, t} \Delta\left(m_{j, t} M_{j, t}\right)-\left(1-\alpha_{N, t}-\alpha_{M, t}\right) \Delta\left(r_{t} K_{j, t}\right), x_{j, t}=$ $\Delta\left(p_{j, t} Y_{j, t}\right)-\Delta\left(r_{t} K_{j, t}\right)$, and $\varepsilon_{j, t}$ the i.i.d. error term. $\Delta\left(p_{j, t} Y_{j, t}\right)$ denotes the nominal output growth in industry $j, \Delta\left(w_{j, t} L_{j, t}\right)$ the nominal labor cost growth, $\Delta\left(m_{j, t} M_{j, t}\right)$ the growth in nominal intermediate input costs and $\Delta\left(r_{t} K_{j, t}\right)$ the nominal capital cost growth. All these variables are compiled from the EU KLEMS database except the user cost of capital $r_{t}$. No sector-specific information was available to construct $r_{t}$, so the rental price of capital is calculated as $r_{t}\left(\equiv r_{j, t}\right)=p_{I}\left(i-\pi_{G D P}+\delta_{K}\right)$, with $p_{I}$ is the deflator for business non residential investment, $i$ the long-term nominal interest rate, $\pi_{G D P}$ the GDP deflator based inflation rate and the depreciation rate is fixed at $5 \%$ throughout $\left(p_{I}, i\right.$ and $\pi_{G D P}$ were taken from OECD database). An econometric issue faced when estimating (186) with the OLS is the potential endogeneity of the regressor associated with the heteroskedasticity and autocorrelation of the error term. To tackle these problems, we estimate (186) by using heteroskedastic and autocorrelation consistent standard errors as suggested by Newey and West [1993] (lag truncation $=2$ ). Finally, the markup estimate $\hat{\mu}_{j}$ is equal to $1 /\left(1-\hat{\beta}_{j}\right){ }^{4}$

\footnotetext{
${ }^{3}$ Austria, Belgium, Denmark, Spain, Finland, France, Germany, Italy, Japan, Netherlands, Sweden, the United Kingdom (UK) and the United States (US).

${ }^{4}$ Countries estimates for each $\hat{\mu}_{j}, j=1, \ldots, 11$, are not reported here to save space, but are available upon request.
} 
Sectoral government expenditure data over the period 1978-2004 were obtained from the Government Finance Statistics Yearbook and OECD database. Following Morshed and Turnovsky [2004], the following four sectors were treated as traded: Fuel and Energy; Agriculture, Forestry, Fishing, and Hunting; Mining, Manufacturing, and Construction; Transport and Communications. The following sectors were treated as being non traded: Government Public Services; Defense; Public Order and Safety; Education; Health; Social Security and Welfare; Housing and Community Amenities; Recreation Cultural and Community Affairs.

Disaggregation of gross fixed capital formation in OECD countries distinguishes between five types of investment inputs. Products of Agriculture, Forestry, Fishing and Aquaculture; Metal Products and machinery; and Transport Equipment are defined as traded inputs. Housing; and Other Buildings are treated as non traded investment goods (source: OECD Input-Output database).

Payroll tax rate, labor income tax rate, and the consumption tax rate are specified as effective tax rates and are computed according to the following formulas:

$$
\begin{aligned}
\tau^{F} & =\frac{\text { Taxes on payroll and workforce }+ \text { Employers' contribution to social security }}{\text { Compensation of employees }}, \\
\tau^{H} & =\text { Income tax (average rate) }+ \text { Employees' social security contributions (average rate), } \\
\tau^{c} & =\frac{\text { Taxes on production, sale, transfer }}{\text { Final consumption expenditure of households and general government }} .
\end{aligned}
$$

Tax allowances, $\kappa$, is calculated as the share of taxable income into the gross wage earnings before taxes. All taxes and incomes data were taken from OECD database.

Finally, data for private consumption, government and investment expenditure, as well those for GDP were drawn from the OECD "National Accounts of OECD Countries" database.

Table (9) summarizes the values of the non tradable share in overall GDP, total employment, public spending, and sectoral output shares of capital income, and, markups. 
Table 9: Ratios for Countries

\begin{tabular}{lcccccccccccc}
\hline & $Y^{N}$ & $\frac{L^{N}}{L}$ & $\frac{I^{N}}{I}$ & $\frac{g^{N}}{Y^{N}}$ & $\frac{g^{T}}{Y^{T}}$ & $\frac{c}{Y}$ & $\frac{g}{Y}$ & $\frac{I}{Y}$ & $\theta^{T}$ & $\theta^{N}$ & $\mu$ \\
\hline \hline Austria & 0.65 & 0.60 & 0.59 & 0.28 & 0.07 & 0.55 & 0.20 & 0.24 & 0.28 & 0.32 & 1.52 \\
Belgium & 0.67 & 0.65 & n.a. & 0.30 & 0.09 & 0.54 & 0.24 & 0.22 & 0.33 & 0.35 & 1.39 \\
Denmark & 0.70 & 0.67 & 0.58 & 0.40 & 0.07 & 0.52 & 0.29 & 0.21 & 0.32 & 0.32 & 1.52 \\
Spain & 0.61 & 0.59 & 0.64 & 0.25 & 0.05 & 0.61 & 0.16 & 0.24 & 0.41 & 0.33 & 1.37 \\
Finland & 0.58 & 0.57 & 0.63 & 0.34 & 0.09 & 0.51 & 0.23 & 0.25 & 0.35 & 0.26 & 1.41 \\
France & 0.69 & 0.64 & 0.63 & 0.33 & 0.06 & 0.56 & 0.24 & 0.21 & 0.27 & 0.30 & 1.42 \\
Germany & 0.64 & 0.61 & 0.61 & 0.30 & 0.06 & 0.57 & 0.22 & 0.23 & 0.22 & 0.35 & 1.55 \\
Italy & 0.63 & 0.56 & 0.54 & 0.29 & 0.06 & 0.58 & 0.20 & 0.23 & 0.22 & 0.33 & 1.73 \\
Japan & 0.64 & 0.61 & 0.59 & n.a. & n.a. & 0.53 & 0.14 & 0.30 & 0.42 & 0.39 & 1.63 \\
Netherlands & 0.67 & 0.69 & 0.63 & 0.34 & 0.08 & 0.50 & 0.25 & 0.22 & 0.37 & 0.29 & 1.36 \\
Sweden & 0.65 & 0.67 & 0.47 & 0.43 & 0.09 & 0.49 & 0.31 & 0.20 & 0.30 & 0.30 & 1.44 \\
UK & 0.62 & 0.66 & 0.52 & 0.33 & 0.05 & 0.60 & 0.23 & 0.19 & 0.30 & 0.28 & 1.47 \\
US & 0.68 & 0.72 & 0.59 & 0.22 & 0.06 & 0.66 & 0.17 & 0.19 & 0.36 & 0.32 & 1.42 \\
\hline \hline Total average & 0.65 & 0.63 & 0.59 & 0.32 & 0.07 & 0.56 & 0.22 & 0.23 & 0.32 & 0.32 & 1.48 \\
$k^{T}>k^{N}$ & 0.64 & 0.64 & 0.60 & 0.30 & 0.07 & 0.57 & 0.20 & 0.23 & 0.37 & 0.31 & 1.44 \\
$k^{N}>k^{T}$ & 0.66 & 0.63 & 0.57 & 0.33 & 0.07 & 0.55 & 0.24 & 0.22 & 0.28 & 0.33 & 1.51 \\
\hline
\end{tabular}

